An Investigation of Executive Function Abilities in Adults with Prader-Willi Syndrome.

Robert M Walley

Doctor of Psychology.

The University of Edinburgh 2005 


\section{Declaration}

I declare that this thesis is my own work and has not been submitted for any other degree or qualification.

R.M.Walley 


\section{Acknowledgements}

I would like to acknowledge the help of the following: -

The PWS and comparison group participants, their families and carers.

My supervisor, Professor M Power, University of Edinburgh for his constructive criticism and feedback on each section of the study.

Dr M D C Donaldson, Department of Child Health, Glasgow University, for his encouragement and support.

Dr A Still, Department of Clinical Psychology, University of Edinburgh, for help regarding statistical matters.

Professor C Temple, Department of Psychology, University of Essex, for giving me a copy of the Self-Ordered Pointing Test.

The author is also grateful to his co-author Dr M.C Donaldson and Blackwell Publishing Ltd for giving their permission to include as appendix B in the thesis the following article which is based on the contents of the thesis.

An investigation of executive function abilities in adults with Prader-Willi syndrome.

R.M. Walley and M.D.C.Donaldson (2005)

Journal of Intellectual Disability Research 49 pp 613-625 


\section{$\underline{\text { Index }}$}

Introduction page 1

Literature Review page 5

Materials and Methods page 42

Discussion page 67

References $\quad$ page 88

Appendix A page 102

Appendix B page 120 


\section{Abstract}

Background

Prader-Willi syndrome (PWS) is a genetic disorder caused by the absence of expression of maternally imprinted genes on chromosome 15 . There are two main genetic sub-types, deletion and uniparental disomy (UPD). In addition to mild/borderline intellectual disability and the almost universal feature of hyperphagia, PWS is associated with high rates of behaviour problems. The present study seeks to explore whether these behaviour problems are associated with relative deficits in executive function (EF), which comprises the non-automatic processes utilized by an individual when faced with a novel situation.

\section{Methods}

Nineteen adult participants with a clinical diagnosis of PWS (12 with deletion subtype, 6 with UPD, and 1 with an uncertain genetic diagnosis) were recruited from a PWS clinic, and compared with 15 participants of similar age and verbal ability on a series of EF tasks and Digit Span Forwards. An informant completed two ratings of behaviour, the Aberrant Behavior Checklist $(\mathrm{ABC})$ and the Dysexecutive questionnaire (DEX).

\section{$\underline{\text { Results }}$}

The PWS group had significantly higher scores on the ABC but not the DEX. There were no significant differences between the whole PWS group and the comparison group on any of the EF tasks, but there was a non-significant trend for the deletion group to show more efficient performance on a planning task. The deletion group was significantly poorer at Digit Span Forwards.

\section{Conclusions}

The lack of relative deficits in EF task performance does not support the hypothesis that EF differences could account for the high levels of behaviour problems found in PWS. Applying the Baddeley and Hitch model of working memory it is suggested the PWS deletion group may have a relative impairment in the capacity of the phonological store. As differences in EF ability were not found, it is suggested that the orbitofrontal cortex, which is involved in the modulation of emotion but not EF, may be implicated in the behaviour problems reported in PWS. 


\section{Introduction}

Prader-Willi Syndrome (PWS) is a genetic syndrome with a distinctive behavioural phenotype characterised by hyperphagia, which persists throughout life and can lead to severe obesity. PWS is caused by a deletion or absence of material on the paternally derived chromosome 15 . In addition to hyperphagia the behavioural phenotype of PWS comprises a number of other behaviours including temper tantrums, obsessive-compulsive behaviours, skin picking, perseverative speech, rigid thinking and difficulties in coping with change. Emotional lability and problems of mood are also frequently noted (Dykens and Cassidy 1996, Einfeld et al 1999). PWS is also usually associated with mild to borderline intellectual disability (ID). For the purposes of the present study the definition of term behavioural phenotype is that given by Dykens (1995, p 528) who suggests that a behavioural phenotype "may best be described as the heightened probability or likelihood that people with a given syndrome will exhibit certain behavioural and developmental sequelae relative to those without the syndrome."

One of the challenges in behavioural phenotype research is to understand the relationship between the genetic disorder and the behaviour characteristic of the syndrome. In doing so it is important to be aware as Hodapp (1997) points out that just as genetic disorders do not have uniform effects on behaviour, behaviour may also be affected by developmental and environmental factors. Similarly the unaffected part of an individual's genome may have a significant affect on behaviour.

As Hodapp suggests and as implied by Dyken`s definition of the term behavioural phenotype it is useful to consider that the effects of a genetic disorder are in some cases specific but more frequently partially specific to a particular syndrome. It is also helpful to think of the genetic effects of a syndrome as comprising direct and indirect effects. Direct effects include the biological interactions between the genetic disorder and brain development; subsequent problems in cognitive and non-cognitive 
functioning might then lead to an increased vulnerability for the development of characteristic behaviours. Indirect effects include the affect of behaviours that an individual shows on their environment, e.g. mother-child and child-sibling interactions. These indirect effects may be particularly salient when the behavioural phenotype includes aggressive and destructive types of behaviours that can cause family stress.

An intermediate stage in the process of analysing the link between the genetic disorder and its behavioural phenotype is the investigation of cognitive and noncognitive processing that might play a part in underpinning the observed behaviours. This type of investigation is important in its own right because it may lead to:

1) Enhanced understanding of the syndrome which is helpful to parents, carers and affected individuals themselves

2) Suggestions regarding the application or development of appropriate psychological approaches to ameliorating the behaviours concerned

3) Indicate how the individuals affected by the syndrome may benefit from particular approaches to learning, which may be important in the context of education and employment

4) Provide suggestions as to which neural pathways maybe involved which could lead to improved pharmacological approaches to treating the behaviour

5) No immediate benefits but rather the generation of further testable hypotheses regarding the relationships between cognitive and non-cognitive processes and the behaviours concerned.

In PWS there seems to be excessive psychopathology relative to individuals with ID generally, especially in respect of anti-social behaviours (Einfeld et al 1999). Waters (1999) notes a picture of social immaturity in PWS contrasted against relatively good cognitive ability, compared to other individuals with ID.

What then would be a starting point for investigating the cognitive processes in PWS which might bear fruit in assisting with an understanding of the behaviours observed and which could build on previous work already carried out? Despite a considerable 
literature on the behaviour problems seen in PWS and more limited studies on the cognitive profile there have to date been very few suggestions of what cognitive or non-cognitive processes may play a part in forming the PWS behavioural phenotype. Holland et al (2003) have recently suggested that the obsessive compulsive behaviours, and temper tantrums found in PWS can be conceptualised as arrested brain development; however they do not suggest which processes or parts of the brain may be involved.

Some of the behaviours seen in PWS particularly the temper tantrums, perseverative speech, rigid thinking and likening for routine suggest that the domain of the executive function (EF) might be useful to explore as these types of behaviour are seen in clinical groups with documented EF deficits, e.g. ADHD (Pennington 2002), brain injury (Evans et al 1997).

$\mathrm{EF}$ has a number of definitions but for the purposes of this introduction can be conceptualised as being the processes that are used by an individual when faced with something novel or unexpected in their environment and which cannot be dealt with by the use of automatic processes.

Denckla (1996 p 266) reminds us that it is important not to make too many extravagant claims for EF and to separate it from the "meta " of concept formation and abstract reasoning. Denckla emphasises the role of EF as being central to but not necessarily higher than other brain processes and makes the analogy of EF being an engineer rather that a theoretical physicist or metaphysical philosopher. Indeed Denckla notes that if EF constructs focussed on the processes of initiate, sustain, inhibit/stop and shift, the controversies regarding the overlap of higher order with central processes could be avoided.

Historically EF deficits have been associated with the functioning of the pre-frontal cortex (PFC), although it has now clearly been established that other brain areas (circuits) are involved. (Cummings 1993). The construct of EF relates to psychological processes but with the advent of more sophisticated scanning 
techniques it has been tempting to investigate the relationship between what are considered to be EF measures and corresponding areas of brain functioning. As will be described in later chapters there are a number of difficulties in assessing EF not least of which concerns the problem of validity of EF measures and the often poor correspondence between EF measures and real life behaviours (Hughes \& Graham 2002). A particular difficulty in investigating EF in participants with ID is that the demands of EF tasks frequently impinge on other cognitive processes, e.g. attention, perception and comprehension. A further difficulty related to research in behavioural phenotypes concerns the appropriate choice of a comparison group. For the purposes of assessing EF it is important to attempt to control for variables, which may affect performance on EF tasks.

Despite the difficulties in assessing EF the premise of this study is that investigating EF in PWS could be a useful contribution to our understanding of the syndrome; the hypothesis being that individuals with PWS will have relative deficits contrasted to a comparison group of similar ability. 


\section{$\underline{\text { Literature Review }}$}

\section{Overview of PWS}

Prader-Willi Syndrome (PWS) is a genetic disorder first described by Prader et al (1956) which affects males and females almost equally. It is characterised by infantile hypotonia and feeding problems in the first year of life, followed by hyperphagia in childhood which if not controlled can lead to severe and potentially life threatening obesity in adulthood. Other characteristics include almond shaped eyes, small hands and feet, endocrine problems affecting growth and sexual development, and developmental delay usually leading to mild or borderline intellectual disability. Holm and Cassidy (1993) describe further minor and supportive diagnostic criteria for PWS, which include behaviour problems, sleep disturbance and speech and articulation difficulties.

A recent UK study (Whittington et al 2001) has estimated that the birth incidence of PWS is around 1:22,000 and the population prevalence between 1:45,000 and $1: 52,000$. Accurate genetic diagnosis is important, because a number of individuals may have the clinical features of Prader-Willi Syndrome, but not the genetic abnormalities associated with it.

PWS is caused by the absence of expression of maternally imprinted genes proximal to the centromere on the long arm of chromosome 15 (15q 11-13) (Cassidy and Schwartz 1997, Webb et al 2002). Because this region of chromosome 15 is maternally imprinted (switched off) PWS will occur if this region of paternal chromosome is deleted, or when the paternal chromosome is absent, and two copies of the maternal chromosome 15 are present (uniparental disomy). The deletion subtype is associated with $70 \%$ of cases of PWS, whilst uniparental disomy (UPD) is found in about $25 \%$ of individuals. About $5 \%$ of PWS is caused by a micro-deletion on the imprinting centre or as a result of a chromosomal translocation. The role of the genes involved in PWS is not yet fully understood, but it is presumed they are important in brain development, and there is particular interest in the development of 
the hypothalamus, which is involved in the regulation of eating, drinking, temperature and sleep (Dykens and Cassidy 1996).

Differences between the deletion and UPD sub-types have been observed. Individuals with UPD appear to have better verbal abilities than those with a deletion and generally seem to have fewer behavioural problems (Dykens et al 1999, Roof et al 2000). The deletion group are believed to have better visuospatial skills and may show splinter skills at jigsaw puzzles, (Dykens 2002), although the latter skill may be due to practice effects (Whittington 2004a). Recent evidence indicates that the individuals with UPD are at high risk of developing an affective psychotic illness in adulthood (Boer 2002).

\section{Behaviour Problems in PWS}

Many of the behaviour problems found in PWS begin in early childhood, and persist through adolescence into adulthood. PWS infants are often described as placid and good-natured but once they have gained reasonable mobility in early childhood, the problem of hyperphagia becomes apparent. It is believed that the hyperphagia is due to impairment in the feeling of satiety so that an individual with PWS because they do not feel full up will carry on eating, and may also not be selective about the type of food that is eaten (Holland et al 1995, Dykens and Cassidy 1996, Akefeldt and Gillberg 1999). Hyperphagia continues to be a major problem in adolescence and adulthood where controls on food are commonly associated with temper tantrums; it is a frequent observation that individuals with PWS are adept at obtaining and finding food even when carers place restrictions on food input. The behaviour problems found in PWS are not always related to food issues and include non-food related temper outbursts, obsessive behaviours, skin picking, perseverative speech, rigid thinking and difficulties in coping with change (Clarke et al 1996, Dykens and Kasari 1997, Einfeld et al 1999). The obsessive behaviours found in PWS tend to be ritualistic behaviours, such as objects having to be in a particular order, hoarding, insistence on routines, and repetitiveness of actions and speech, rather than typically obsessive compulsive symptoms such as checking, cleaning, or obsessional thoughts. Whilst temper outbursts and ritualistic behaviours are found in typically developing 
children, in PWS they continue well beyond the developmental stage at which they would be considered appropriate, and persist into adolescence and adulthood (State et al 1999, Clarke et al 2002, Wigren and Hansen 2003).

The behaviour problems found in adults with PWS can be difficult to manage. Temper outbursts and arguments about changes in routine may be a particular source of stress within a family setting (Sarimski 1997, Donaldson 2002).

\section{Maladaptive Behaviours}

Clarke et al (1996) compared thirty PWS adults, age range 16 to 44 , with thirty adults who had similar levels of ID, using the Aberrant Behaviour Checklist (ABC) (Aman et al 1985), a fifty-eight item standardised questionnaire completed by an informant who knows the individual well and which in addition to a total score yields scores for five factors; i) irritability, agitation ii) lethargy, withdrawal iii) stereotypic behaviour, iv) hyperactivity, non-compliance, v) inappropriate speech; the $\mathrm{ABC}$ has excellent psychometric properties.

The results of this study suggested that temper tantrums, self-injury, impulsiveness, lability of mood, inactivity and repetitive speech are characteristic behaviours of PWS. The factor i) scores in the PWS sample were found to be as high as those of inpatients in hospital for adults with ID. In addition to having significantly higher factor i) scores the PWS group also were found to have a significantly higher score on factor v) (inappropriate speech). This study found no relationship between the factor scores and gender; nor was there a linear relationship between age or body mass index (BMI). However the highest factor i) scores were found in the most overweight participants. Weaknesses of the study acknowledged by the authors included low participant numbers and difficulty in matching PWS participants with the comparison group; specifically the variables of obesity and sleep disorders should have been taken into account in the matching process.

A further study by Clarke and Boer (1998) used the ABC to investigate differences between participants with the deletion subtype of PWS, Smith-Maginnis and Cri du 
Chat syndromes. All three of the syndromes shower elevated rates of behaviour problems, but the PWS group was characterised by temper tantrums, inability to delay gratification, repetitive speech, lability of mood, inactivity and self-injury. The most problematic behaviours shared by PWS and Smith-Maginnis groups and demonstrating partial specificity were impulsiveness and mood instability. The authors however noted that whilst temper tantrum behaviour has a very strong association with PWS, it is not the case that every individual with PWS shows this behaviour and the severity of temper tantrums may vary over time. More recently Clarke and his colleagues (Holland et al 2003) have used the ABC with a relatively large group of sixty-five individuals with PWS who were identified in a populationbased study. Whilst the PWS group had higher scores than an ID comparison group on all the factor scores these only reached significance for the overall score and the irritability scale (factor i). Analysis indicated that the PWS group were more likely to have problems relating to: - temper tantrums, demands must be met immediately, inactivity, repetitive speech, not attending to instructions, distractible and tantrums if doesn't get own way. In contrast to Clarke et al (1996) this study found that the scores on the $\mathrm{ABC}$ increased with age but decreased with increased IQ in PWS adults. Aberrant behaviour was associated with the severity of the eating disorder.

Similar types of behaviour problems have been observed using different behaviour checklists to investigate the PWS behavioural phenotype. Dykens and Kasari (1997) compared the maladaptive behaviour of forty-three children with two age and gender matched comparison groups, one comprising children with Down syndrome, the other non-specific ID. In this study the Child Behaviour Checklist (Achenbach 1991), a 112 item standardised behaviour checklist, was used. The Child Behaviour Checklist measures externalising problems including delinquent and aggressive behaviours and internalising problems including withdrawn, somatic complaints, anxious/depressed behaviours. It also measures social problems, thought problems, attention problems and other problems. Dykens and Kasari found that the PWS group exhibited a distinctive set of behaviours in addition to having higher scores on both the externalising and internalising problem domains. The set of behaviours distinctive to PWS included over eating, food obsessions, and sleep disturbance in addition to repetitive and compulsive-like behaviours and talking too much. A 
weakness of the study was that the three groups were not matched for levels of obesity although no relationships within the participant groups were found between BMI and scores on the checklist. This study noted that previous research (Dykens and Cassidy 1995) had shown that in adults with PWS a lower BMI was associated with internalising difficulties such as depression, anxiety, distress and distorted thinking, relative to adults with a higher BMI.

Dykens et al (1999) has also used the Child Behaviour Checklist to investigate differences between the deletion and maternal disomy subtypes of PWS. In this study there were twenty-three participants in each group ranging in age from 6 to 42 years. The study indicated that the deletion group showed more problem behaviours than the UPD group and were more likely to overeat, self-injure, hoard, sulk and withdraw.

Further converging evidence for a distinctive pattern of maladaptive behaviours in PWS is provided by studies that have utilised the Developmental Behaviour Checklist (Einfeld and Tonge 1994, 1995), which is a ninety-six-item informant rated questionnaire available in a parent and teacher version. It was designed for use with children and young people but has been used with adults; like the $\mathrm{ABC}$ it has excellent psychometric properties. In addition to a total score it provides information on six-sub scales, disruptive, self-absorbed, communication disturbance, anxiety, autistic relating and anti-social. Einfeld et al (1999) using the DBC asked the parentcarers of forty-six children with PWS to provide information about their behaviour. The study revealed that compared to a matched comparison group of children with ID the PWS group had a significant higher level of psycho-pathology and in particular had higher scores on the anti-social sub-scale. In terms of individual items of the DBC the PWS group scored significantly higher on temper tantrums, gets obsessed with an idea or activity, scratches or picks skin, eats non-food items, gorges food and shows mood changes rapidly for no apparent reason.

Holland et al (2003) in their population-based study used the DBC on the thirty-three children with PWS who participated but did not find any significant differences from 
the comparison group. There was however a tendency for the PWS group to score more highly on the communication disturbance and autistic traits scales. For individual items the PWS group had an average score exceeding one on distractible, temper tantrums, no sense of danger and stubborn, ordering, obsessive, repetitive speech, routine, poor peer relationships and skin picking.

The DBC has also been used by Steinhausen et al (2004) to investigate behavioural and emotional characteristics of fifty-eight participants with PWS ranging in age from 2 to 29 years and divided into three age groups - pre-school children aged 2 to 6 years, school age children 7 to 13 years and adolescents and young adults aged 14 to 29 years. The study demonstrated an increase in behavioural problems with age. Gender and intelligence was not associated with behaviour problems but increasing BMI was associated with increased risk of behavioural and emotional disturbance. The observation that behaviour problems appear to be associated with increasing age in PWS may account for the differences that Holland et al (2003) found in their study between child and adult participants although they were using different instruments for the two age groups. Steinhausen et al suggest that their study shows adolescents and early adulthood are the critical periods for an increase in behavioural and emotional disturbances in PWS.

The finding of increased behavioural problems in adolescents is also supported by a study that examined the emergence of temper tantrums and compulsive behaviour in children with PWS (Dimitropoulos et al 2001). The study found that although the emergence of temper tantrums is delayed in PWS compared to typically developing children, more severe tantrum behaviour is found in older children with PWS compared not only to typically developing children but also children with Down syndrome. They suggested that in PWS the tantrum behaviour might follow a different developmental trajectory.

A study by Akefeldt and Gillberg (1999) that used a variety of measures (but not the $\mathrm{ABC}$ or $\mathrm{DBC}$ ) also found that in a group of forty-four individuals with PWS ranging in age from 1 to 53 years age was related to the severity of behaviour problems. 
Compared to an ID group who were also matched for obesity, individuals with PWS over the age of seven years showed a distinctive behavioural pattern comprising skin picking, ritualism, old fashioned and precocious behaviour, preoccupation with food and very changeable mood. These authors noted a tendency for participants with PWS to fly off the handle and have temper tantrums when routines changed. Significantly in this study where the comparison group was matched for obesity no evidence was established for an association between weight and behaviour problems; unfortunately not all studies involving PWS control for obesity as a variable.

\section{$\underline{\text { Obsessive- Compulsive Behaviours in PWS }}$}

Compulsive type behaviours have been noted in many of the studies investigating maladaptive behaviour in PWS but a number of studies have focused on this aspect of behaviour and because of its relevance to EF these will briefly be reviewed.

The study by Dimitropoulos et al (2001) has already been mentioned in relation to temper tantrums but also investigated the emergence of compulsive type behaviours in PWS. It found that although PWS children at ages 2 to 3 were similar to typically developing children, by the ages of four to six they were displaying much higher rates of compulsive type behaviour. The behaviours described included ordering and arranging activities and displaying a need for balance and symmetry. This study suggested that the compulsive behaviour seen in older PWS children was not linked to their eating disorder; it also observed that in addition to non-food compulsions many children with PWS engage in skin picking between the ages of four to five.

Dykens et al (1996) investigated obsessions and compulsions in 91 people with PWS ranging in age from 5 to 47 years (mean age 19 years) and found evidence of compulsive behaviours seen in younger children. Prominent compulsive symptoms present in $37 \%$ to $58 \%$ of the PWS participants included hoarding, ordering and arranging, concerns with symmetry and exactness, rewriting and needs to tell, know or ask. This study used a standardised obsessive-compulsive scale but did not include an ID comparison group. However it did include a group of forty-three adults without ID who had a diagnosis of obsessive-compulsive disorder (OCD). The 
authors report that the PWS and OCD groups were similar in terms of symptoms severity and number of compulsions.

Clarke et al (2002) in their population based study examined compulsive and ritualistic behaviours in the sixty-five PWS participants by using a semi-structured interview with their carers/parents rather than a standardised checklist for obsessivecompulsive symptoms. They felt this method was more suitable given the questionable validity that the majority of standardised checklists have in respect of their use with an ID population. As in other studies Clarke et al found that compulsive symptoms did not decline with age nor were they correlated with obesity, severity of eating behaviour or anxiety/depression. They found that there were few obsessive thoughts reported and the range of compulsive symptoms was relatively restricted. They noted that PWS is associated with high rates of ritualistic behaviours such as the need to ask or tell something, insistence on routines, hoarding and ordering objects and repetitive actions and speech. They also noted that although compulsive behaviours were common in typically developing children at age eight years they were uncommon beyond the age of thirteen; the pattern of compulsive behaviours therefore seen in adults with PWS is more similar to that seen in younger typically developing children than in typically developing adolescents and adults diagnosed with OCD.

Wigren and Hansen (2003) carried out a study on fifty individuals with PWS using a standardised instrument to measure compulsive behaviour in children, which provides further confirmatory evidence that the compulsions of childhood do not subside with age in PWS. Importantly in respect of the current study they note that "compulsive like behaviours are generally considered to help the child to master anxiety by keeping some degree of control in an ever changing environment" (p430). Wigren and Hansen found that the PWS group displayed higher rates of behaviour patterns related to insistence on sameness in daily routines and activities which did not decrease with age. They went on to point out that after the age of five typically developing children showed decline in compulsive behaviour because of a diminishing need for predictability and control of external events. 


\section{Motivation in PWS}

A study by Dykens and Rosner (1999) investigated the personality-motivation in thirty-five individuals with PWS using a motivation questionnaire standardised for use in people with ID and completed by an informant. The age range of PWS participants was between 14 and 50 and they were contrasted with a similar size group of individuals with Williams syndrome and a group of individuals with nonspecific ID. Not surprisingly they found the participants with PWS were motivated by food and also they were motivated by orderliness. This finding is consistent with the pattern of compulsive behaviours found in PWS described above. This study also showed that PWS participants were more likely to enjoy puzzles or mysteries that as will be discussed later in this review may be linked to particular cognitive strengths in visual processing.

\section{Sleep and Behaviour Disturbance in PWS}

As briefly noted in the introduction to the review, sleep disturbance is a feature of PWS and may be a factor contributing to the other behaviours problems characteristic of the syndrome. Richdale et al (1999) investigated this possibility by asking the parent/carers of twenty-nine individuals with PWS to complete a number of questionnaires related to sleep as well as the DBC. They also asked parents/carers of a comparison group matched for gender, age and ID to complete the questionnaires. The age range of the PWS group was six months to forty-six years. The study found that the PWS group were highly likely to display excessive day time sleepiness (EDS) and were also more likely to develop snoring, and early morning waking. In relation to behaviour the study found that susceptibility to EDS was associated with increasingly maladaptive behaviour. Taking into account earlier work the authors questioned whether there might be a relationship between poor quality sleep, EDS and the irritable/fractious behaviour commonly observed in PWS. However they concluded that further work in this area was needed to explore these relationships before any conclusions regarding their direction could be provided. The authors also suggested that EDS may be a specific characteristic of PWS and might 
be related to control of the sleep-wake process, which is associated with the functioning of the hypothalamus.

\section{$\underline{\text { Social Behaviour }}$}

Surprisingly given the extensive literature on maladaptive behaviour in PWS there is little published research into social behaviour. Despite having relatively mild levels of learning disability, individuals with PWS find it difficult to establish peer group relationships and have been described by teachers as socially immature (Waters 1999). Holland et al (2003) found that their PWS group had a relative strength in the daily living skills domain of the Vineland Adaptive Behaviour scales (Sparrow et al 1984), but a relative weakness in the socialisation domain, confirming similar results obtained by Dykens (1992a). Whittington et al (2004b) found that academic under-achievement was negatively correlated with scores on the Vineland socialisation domain.

\section{Ageing and Behaviour}

There is a high mortality rate in PWS, estimated at 7\% per year for adults aged 30 years and older (Whittington et al 2001) associated with complications arising from obesity; because of this the majority of studies investigating behaviour in PWS are not able to include many adults over the age of 30 .

Dykens (2004) has been able to investigate behavioural differences between 45 adults with PWS aged between 30 and 50 years and 195 children, adolescents and young adults by recruiting participants from a number of sources in the USA. As in previous studies behavioural problems were particularly problematic in young adults aged 20 to 29 years, however in the older adult group it was found that maladaptive and compulsive symptoms had decreased significantly. Dykens suggested a number of possibilities for this decline of maladaptive behaviour in the older age group including selective survival issues and unknown hormonal or physiological factors. She also suggested that long-term exposure to behavioural and dietary interventions may have been responsible. 


\section{Mental IIlness in PWS}

The purpose of the current study is to investigate the possible relationship between $\mathrm{EF}$ and behaviour in PWS and is not directly concerned with mental illness. However as EF problems have been implicated in studies of mental illness (e.g. Evans et al 1997) its association will be reviewed briefly here.

Clarke et al (1998) reviews the literature on mental illness in PWS and describes six cases of individuals who develop psychoses. Clarke et al noted that individuals with ID are more vulnerable than the general population to developing mental illness but it appears that individuals with PWS have a particular vulnerability to develop psychotic symptoms in adult life. It has often been difficult to classify the illness but both Clarke and Verhoeven et al (1998) note that cycloid psychosis has been found in PWS. Verhoeven et al also note that mood and anxiety dominate the psychiatric symptomology of PWS.

Beardsmore et al (1998) reviewed the psychiatric diagnoses of twenty-three adults with PWS living in a particular area who were already known to psychiatric services. They found that although the PWS group had similar rates of schizophrenia/ delusional disorders to a comparison group, they had higher rates of affective disorders in which psychotic symptoms were common. Interestingly none of the PWS group were found to have generalised anxiety or phobic disorders.

Boer et al (2002) in an epidemiological study involving sixty-five individuals with PWS ascertained that fifteen adults participating in the study had a psychiatric disorder. Seven of the fifteen adults had a severe affective disorder with psychotic symptoms; of the seven five had the maternal disomy sub type of PWS. This study concluded that although deletion sub type of PWS is associated with slightly higher rate of psychotic illness - $8 \%$ - compared to the $5 \%$ normally reported for ID there was evidence of a very strong $100 \%$ occurrence of psychotic illness in older adults with UPD. 


\section{Cognitive Ability in PWS}

The profile of general intellectual functioning (IQ) in PWS is usually one of mild to borderline intellectual disability, but some individuals have an IQ in the average range. Curfs and Fryns (1992) carried out a meta-analysis of fifty-seven studies investigating IQ in PWS and reported that $4.9 \%$ of individuals with PWS were in the normal range of intelligence; $27.8 \%$ were in the borderline range, $34.4 \%$ in the mild range, $27.3 \%$ in the moderate range and $5.6 \%$ in the severe-profound range of ID. Dykens et al (1992b) carried out a longitudinal study of IQ in PWS and found no evidence for a decline in intelligence over time.

Roof et al (2000) have found that individuals with UPD have a higher verbal IQ than individuals with a deletion. They also found that levels of performance IQ were similar for both groups but that in the deletion group performance IQ was likely to be slightly higher than verbal IQ. In their study Roof et al using the appropriate Wechsler IQ scales assessed 38 individuals with PWS, 24 with the deletion and 14 with UPD. The mean age of the groups were 21.8 and 22.8 years respectively and the age range was 10 to 44 years. The mean verbal IQ for the deletion and the UPD groups were 60.8 and 69.9 respectively, performance IQ was 64.7 and 62.2 and full scale IQ was 61 and 64.1 .

Whittington et al (2004a) assessed IQ in 92 individuals with PWS using the appropriate Wechsler scales as part of a larger population based study, which for the purposes of this part of the study also included individuals with PWS from outside the region concerned. They also found that the UPD group had better verbal abilities than the deletion group, but noted relative weakness in coding ability (one of the Wechsler sub-tests) in the UPD group; no specific gender effects were found. Whittington et al (2004a) report that the mean verbal IQ for the entire PWS group was 66.9; performance IQ 64.1 and full scale IQ 63.6. The mean verbal IQ for the deletion and the UPD group separately was 65.8 and 69.7 respectively, performance IQ 65.9 and 61.05 , full scale IQ 61.6 and 63.3. Whittington et al noted that the deletion group were similar in performance to the ID comparison group used. Other studies investigating cognitive abilities in PWS, have found that individuals with 
PWS tend to perform better on tasks requiring visual processing skills than tasks that require auditory processing.

Gabel et al (1986) used a number of adult neuropsychological tests to assess the abilities of fifteen children but used as a comparison group typically developing children matched only for age and sex. Not surprisingly the PWS group did much more poorly than the comparison group, however the authors observed that the PWS group were relatively poorer on tasks requiring use of the auditory modality.

Curfs et al (1991) analysed the sub tests scores of Wechsler intelligence tests given to a group of twenty-six children and adolescents with PWS ranging in age from 7 to 15 years. They observed that visuo-spatial skills appeared to be a relative strength but that the PWS participants had particular difficulty on two sub tests - arithmetic and digit span which required auditory processing abilities.

The finding that individuals with PWS have relative strengths in visuo-spatial abilities has been associated with the observation that they appear to have a particular talent for completing jigsaw puzzles. Holm et al (1993) on the basis of clinical impressions included special skills at jigsaw puzzles as a supportive diagnostic criterion for PWS. Dykens (2002) has set out to investigate whether these clinical impressions are valid by testing PWS children on a number of standardised visuospatial tasks and two specially designed jigsaw puzzles. Dykens compared their performance with a typically developing group of children as well as a group matched for ID. In the introduction to her paper Dykens suggests "Puzzle skills may take advantage of the visual motor strengths shown by many with the syndrome, as well as their obsessive compulsive tendencies and needs for order, exactness and for things to be just right" (p343). The study found that the PWS group were better than the ID comparison group at visuo-spatial tasks but not as good as the typically developing group, however their performance at jigsaws exceeded both other groups. This led Dykens to suggest that jigsaw puzzles may be a spared or splinter skill in PWS. Interestingly this finding only appeared to apply to individuals with the deletion type of PWS. The small number of participants with UPD did not show the 
same relative abilities at visuo-spatial tasks or particular skills at jigsaws. Dykens also noted that the overall ability of the PWS group at jigsaw puzzles was not related to IQ or compulsive behaviours. It was however modestly correlated with age and Dykens acknowledged that the fondness PWS children have for jigsaw puzzles may have affected the study findings.

The results of Dykens study have been disputed by Whittington et al (2004a) who in their population based study examined performance and visuo-spatial tasks with individuals with deletions and UPD reported as having exceptional skills at jigsaw puzzles and also those said not to have jigsaw puzzle skills; the comparison ID group were also included in this analysis but none of them were said to have exceptional jigsaw skills. In contrast to Dykens study Whittington et al found that relatively better visuo-spatial skills were only observed in the deletion group who were reported to have exceptional jigsaw puzzle skills and suggested that the ability to do jigsaw puzzles as noted by Dykens might well be due to practice effects. Overall the PWS group did less well on visuo-spatial tasks than the ID group.

\section{Explanatory Mechanisms for the Behaviours observed in PWS}

The emphasis of explanatory mechanisms for the behaviours observed in PWS has been on biological rather than psychological processes.

State et al (1999) note that no distinct brain areas have been identified as grossly pathological by neuroimaging procedures, but believe that because of the problems of growth, sexual maturation, sleep and temper regulation, the hypothalamus is involved. To support this view State et all review evidence concerning the hormone oxytocin which is important in grooming behaviours and food satiety; they cite the work of Swaab (1995) who found a decreased number of oxytocin neurons in the hypothalamic paraventricular nuclei of five post-mortem PWS brains.

Dykens et al (1999) suggest that serotoninergic and dopaminergic mechanisms are implicated in the compulsive behaviour seen in PWS, which can be viewed as grooming and nesting behaviours that have gone awry. Clarke et al (2002) also 
suggest a role for the involvement for serotoninergic systems in the compulsive behaviours found in PWS.

Richdale (1999) has as discussed above also suggested that abnormalities in the hypothalamus are implicated in the excessive daytime sleepiness characteristic of PWS and also that poor sleep quality in PWS may have an effect on behaviour.

The cognitive profile of PWS indicates that brain regions other than hypothalamus may be characteristically different compared to other learning disability populations. Dykens (2002) has put forward the hypothesis that the superior jigsaw puzzle skills in PWS may be related to the functioning of visual processing systems involving the superior parietal cortex and/or the ventral posterior cortex. The finding of better visual processing than auditory processing in PWS is also supported by an event related potential (ERP) study (Stauder et al 2002).

A single case study utilising 3D MRI on a 40-week-old girl with PWS found evidence of diffusely abnormal cerebral gyral folding suggestive of a cortical malformation (Yoshii et al 2002).

Lucignani et al (2004) have found reduced gamma-aminobutyric acid type A (GABAa) receptor activity predominately in the limbic and frontal cortex of six individuals with deletion type PWS compared to nine comparison participants using a positron emission tomography (PET) approach. It was not entirely clear in the paper but it would appear that the comparison group were not matched for IQ.

Holland et al (2003) have proposed that some of the behaviours in PWS, particularly compulsive behaviours and temper tantrums, may be caused by arrested brain development. Factor analysis on their behavioural data indicated presence of three factors, which correspond to eating, personality and emotions.

The eating factor includes eating related behaviour together with lying and stealing (often related to food); the second factor, personality, includes obsessive behaviours, 
temper tantrums, aggressive/violent behaviour and possessiveness. They note that temper tantrums are often caused by the thwarting of compulsive behaviour, breaking of a schedule or not keeping promises; the third factor includes mood swings, skin picking and argumentativeness.

Holland et al suggest that the three factors have a different neurobiological basis and might correspond to the failure of expression of different imprinted genes in the PWS critical region.

\section{Executive Function}

The literature on the behavioural and cognitive profile of PWS is not currently able to explain why a genetic syndrome that is characterised by relatively mild levels of ID is associated with such high levels of psychopathology. As was discussed in the introduction it may be helpful to explore the domain of EF in PWS to establish whether deficits in this area contribute to the behaviours characteristic of the syndrome. In this part of the review the meaning of the term EF will be expanded and some of the studies in which EF deficits have been found in different clinical groups described. Working memory, because of its importance as an aspect of EF, will be discussed in more depth; working memory has also been relatively well researched in ID compared to other aspects of EF. The review will also include discussion of the measurement problems in EF alluded to in the introduction. The development of EF skills with age will also be considered and some relevant reviews and research studies in this area will be described.

\section{Definitions of EF.}

Hughes and Graham (2002) remind us that the distinction between automatic and controlled actions is fundamental to an understanding of EF; they note that the distinction rests upon three important features:

"the execution of novel vs. familiar action sequences, making a choice between alternative responses vs. the execution of a single action sequence, and the execution of acts that are/are not accessible to consciousness" (p131).

Hughes and Graham do not regard EF as a single process but rather an: 
"umbrella term for all of the complex set of cognitive processes that underlie flexible goal directed responses to novel or difficult situations" (p131)

Norman and Shallice $(1980,1986)$ and Shallice and Burgess (1991) specify that EF processes are required in the following situations: -

1. those that involve planning or decision making

2. those involving error correction or troubleshooting

3. situations where responses are not well learned or contain novel sequences of actions

4. dangerous or technically difficult situations

5. situations which require the overcoming a strong habitual response or resisting temptation.

Welsh and Pennington (1988) highlight the role of a central processing system in their definition, which suggests that $\mathrm{EF}$ is:

"The ability to maintain an appropriate problem solving set for attainment of a future goal. This set can involve one or more of the following: a) an intention to inhibit a response or to defer it to a later more appropriate time, b) a strategic plan of action sequences and c) a mental representation of the task, including the relevant stimulus information encoded into memory and the desired future goal state. In cognitive psychology the concept of EF is closely related to the notion of a limited capacity central processing system" (p201-202).

Pennington and Ozonoff (1996) suggest that EF includes:

"set shifting and set maintenance, interference control, inhibition, integration across space and time and working memory" (p55). They also suggest that a central idea of $\mathrm{EF}$ is "maximal constraint satisfaction in action selection which requires integration of constraints from a variety of other domains, such as perception, memory, affect and motivation. Hence much complex behaviour requires executive function especially much human social behaviour" (p55).

As discussed in the introduction, Denckla (1996) emphasises the central role of EF but in contrast to Pennington and Ozonoff restricts EF: 
"to the more cold cognition aspects of frontal-cortical circuits, leaving the socialemotionality or motivational personality aspects more affiliated with orbital and mesial prefrontal regions out of consideration" (p264).

Denckla's views on this are born out by scanning studies. Duncan and Owen (2000) reviewing neuroimaging studies of the prefrontal lobes report that EF processes recruit the involvement of the mid-dorsolateral, mid-ventrolateral and dorsal anterior cingulate cortex but not the medial or orbital surfaces.

There is insufficient space to describe here all the instruments that have been developed as EF measures but some of them will be mentioned in the following discussion concerning EF deficits that have been found in some clinical groups.

\section{EF Deficits in Clinical Groups}

EF deficits have been found in a diverse range of clinical groups and to describe the relevant literature is beyond the size and scope of the current study. Instead discussion will focus on a study of EF deficits in Turner syndrome, the methodology of which has been adapted for the present study. There will also be a brief discussion of EF studies in another genetic syndrome, Fragile $\mathrm{X}$, as well as autistic spectrum disorder (ASD), attention deficit, hyperactivity disorder (ADHD) and schizophrenia.

\section{EF Deficits in Turner Syndrome}

Turner syndrome is a genetic disorder affecting only girls and results from the absence of or dysfunction in the second X chromosome. It has a distinctive physical phenotype including short stature, and interestingly although it only affects girls is similar to PWS in that the foetus develops with reduced exposure to endogenous sex hormones.

Turner syndrome has a cognitive profile suggesting right parietal lobe damage but a few studies have hinted at the possibility of EF deficits. Temple et al (1996) decided to investigate if indeed EF deficits were evident in the syndrome. They compared 16 participants with Turner syndrome mean age 10.5 years with a group of 18 girls 
mean age 10.3 years. Both groups were of average IQ but were not specifically matched for IQ, as there is evidence of verbal - performance difference in Turner syndrome.

Temple et al (1996) investigated EF using five well known and established EF tests; the Wisconsin Card Sort Test (WCST) (Grant and Berg 1948,Heaton 1981), the Stroop Task (Stroop 1935), Verbal Fluency Tasks (Milner 1964, Newcombe 1969, Thurstone 1938), The Tower of London (Shallice 1982), and The Self Ordered Pointing Tasks of Petrides and Milner (1982).

The Wisconsin Card Sort Test (WCST) requires participants to match a set of sixty four cards which have different shapes printed on them in varying numbers and colours with four key cards differing in colour, form and number. The examiner informs the participant if a correct match has been made and changes the sorting principle after ten correct responses. Participants only become aware that the sorting principle has changed by listening to the feedback of the examiner. The sorting principle is in the order, colour, form, number and is repeated until all the cards are used up. The test is scored on the basis of the number of correct responses made, sorting categories completed (i.e. sets of ten) and the number of perseverative errors made. This test assesses the EF abilities of working memory, inhibition, and the ability to deal with change (change set).

The Stroop task requires participants first of all to read vertical rows of randomly assorted colour words and then to name the colour the words are printed in which are different from the word itself, e.g. if the word green is printed in red, the correct response would be red.

This task is a useful measure of sustained attention but from an EF perspective measures the ability to inhibit a pre-potent response in the absence of feedback.

Verbal fluency tasks require participants to generate as many words as possible in a minute beginning with a particular letter; in the Temple et al study the commonly used letters F, A and S were used. Words with the same stem were only allowed 
once. Participants were also asked to generate words from three semantic categories, animals, objects and occupations; again only a minute was allowed for each category. Scores are based on the number of words generated for each letter or category.

The Tower of London task (TOL) measures planning ability and consists of a board with three pegs of decreasing size upon which can be placed three, two, or one differently coloured balls. A total of three balls were used in this version of the task and from an initial starting position participants had to move balls to an end position printed on a card. Depending on the end position this could be accomplished in two, three, four, or five moves. Four trials (different problems) of each move level were given. Participants are only allowed to move one ball at a time and once a ball is picked up it has to be placed on a peg; balls cannot be held in the hand or put on the table while another move is made. The score is derived from the number of problems correctly solved.

The Self-Ordered Pointing Task used in the Temple et al study was a modification of the original Petrides and Milner task. The task assesses the ability to organise on a time line in working memory a set of visual stimuli. It consists of two parts one comprising visual stimuli made up of concrete items and the other comprising abstract visual stimuli. In each part of the task participants are initially given a set of six pages on which six stimuli are printed in different spatial locations on each page. As they turn a page participants have to point to a different stimulus. This process is repeated for eight ten and twelve pages. The task is scored by totalling the pages before an error was made, the number of times a point is made to the same stimulus (item perseveration) and the number of times a repeated point is made to the same spatial position (position perseveration).

Temple et al found that the Turner syndrome participants showed poorer performance relative to the comparison group on only three of the EF tests, the Stroop, verbal fluency and the abstract version of the Self-Ordered Pointing Task. Temple et al argued that this supported the view that there may be fractionation of 
EF systems in development and that in Turner syndrome planning, changing set and working memory abilities are better developed than the ability to search vocabulary in an organised way and to inhibit a pre-potent response when no feedback is available.

\section{EF in Fragile-X Syndrome}

Fragile- $\mathrm{X}$ syndrome is the most common genetically inherited cause of ID. It is caused by a fault on an $\mathrm{X}$ chromosome; therefore it affects males more severely than females as males only have one X-chromosome. EF has been investigated in a number of studies because of the apparent overlap of Fragile-X syndrome with autistic spectrum disorder which is associated with EF deficits and because of it's association with impulsive behaviour and problems of attention.

Garner et al (1999) used the WCST to investigate whether Fragile-X males without a diagnosis of autism would have EF deficits. Unfortunately both the Fragile $-\mathrm{X}$ group and a comparison group of males with ID performed at floor level on this test so the authors were unable to arrive at any conclusions beyond noting that EF deficits may be a feature of ID in general. Munir et al (2000) using two sub tests from the Test of Everyday Attention For Children (Manley et al 1999) found however that boys with Fragile-X have relative impairment in inhibition compared to a group of boys with Down syndrome and two groups of boys matched for mental age but differing in reported levels of attentional ability, one group having poor attentional abilities and the other group average attentional ability. They also found that the Fragile-X group showed specific deficits in selective and divided attention. This piece of research was further developed by the same authors (Wilding et al 2002) who compared Fragile-X males with a Down syndrome group on a computerised visual search task, the Wilding Attention Test For Children (Wilding 1997). This study found that Fragile-

$\mathrm{X}$ males have a particular difficulty in switching visual attention in addition to a weakness in inhibition. 


\section{EF deficits in Autistic Spectrum Disorder (ASD)}

The adherence to rules and the perseverative behaviour observed in individuals with ASD has led many researchers to believe that EF deficits are fundamental to an understanding of this disorder.

Pennington and Ozonoff (1996) in a major review of EF deficits in developmental disorders noted that in fourteen studies investigating EF deficits in ASD, thirteen of them showed that individuals with ASD differed from the comparison groups on at least one EF measure. The findings of many of these studies support the suggestions that individuals with ASD find it difficult to disengage from immediate environmental cues and use internal rules or mental representations to guide behaviour.

\section{$\underline{\text { EF Deficits in Attention Deficit Hyperactivity Disorder (ADHD) }}$}

The symptoms of ADHD include hyperactivity, impulsivity and distractibility and there has been a substantial literature on investigations of EF deficits in this disorder. In their review of EF deficits in developmental disorders, Pennington and Ozonoff (1996) summarise eighteen studies investigating EF deficits in ADHD, fifteen of these studies showed that individuals with $\mathrm{ADHD}$ performed more poorly on $\mathrm{EF}$ tasks than the comparison groups. Sixty different measures were used across the studies and on forty of these the performance of ADHD participants was relatively poorer. The ADHD participants did not do better on any of the measures. Analysis of these studies by the authors concluded that individuals with ADHD are more likely to show deficits in motor inhibition rather than set shifting or working memory.

More recently this finding has been supported by research utilising the Stop Signal paradigm in which a participant was taught a particular response and is then told to inhibit this response when signalled (by a beep). Oosterlaan and Sergeant (1998) in a meta-analysis of studies using this task found consistent deficits in ADHD participants. Despite the converging evidence of EF deficits playing an important role in $\mathrm{ADHD}$, Pennington (2002) notes that $\mathrm{EF}$ deficits are not able to account for 
all the behaviour seen in the disorder and there are competing explanations for the underlying deficit.

\section{EF Deficits in Schizophrenia}

Frith (1992) has proposed that three major cognitive abnormalities could underlie the major signs and symptoms of this devastating mental illness; a disorder of willed action, a disorder of self-monitoring and a disorder of monitoring the intentions of others. Frith argued in particular that the disorder of willed action could account for symptoms common to both schizophrenia and patients with frontal lobe lesions, e.g. lack of volition and perseveration. Many studies have demonstrated EF deficits in schizophrenia but one of the difficulties has been to disentangle specific EF deficits from the more general cognitive impairment that can accompany the illness; studies that have attempted to do this have produced conflicting findings (Evans et al 1997). Evans et al (1997) have suggested that these conflicting findings may be associated with a lack of sensitivity of the EF tasks used and may have little relationship to executive dysfunction problems in everyday life. To remedy this Evans et al tested a group of schizophrenic patients and a brain injured and healthy comparison group with the Behavioural Assessment of Dysexecutive Syndrome (BADS) (Wilson et al 1996) which is a battery of tests designed to assess EF in a way that is ecologically valid. Evans et al found that the schizophrenic and brain injured participants were equally impaired. Impairment was also present in the sub group of the schizophrenic patients with otherwise intact intellectual abilities. Although many patients with schizophrenia have problems with planning, switching task and problem solving in novel situations, no specific profile of EF deficits was found; this probably reflects heterogeneity of the illness.

Hutton et al (1998) have investigated EF in first episode patients with schizophrenia using the Cambridge Neuropsychological Test Automated Battery (CANTAB) (Sahakian and Owen 1992), a series of computerised tests. Sub tests of this battery measure spatial working memory, planning and attentional set shifting. The participants were also given verbal fluency tasks and other cognitive tasks to measure memory problems. In comparison with a group of healthy volunteers the 
schizophrenic patients had severe impairments in EF, particularly evident for planning ability and strategy use.

\section{Working Memory}

Working Memory can be defined as the limited capacity memory system responsible for the temporary storage and processing of information while cognitive tasks are being carried out. One theoretical model of working memory is that proposed by Baddeley and Hitch (1974) and Baddeley (1986) and comprises a controlling central executive which is modality free and two slave systems which provide temporary storage and maintenance of information. These are the phonological loop and visuospatial sketchpad. The visuospatial sketchpad stores and maintains visual information and the phonological loop has a similar function for verbal material. The phonological loop consists of two sub systems, a phonological store and an articulatory rehearsal process.

This theoretical model of working memory has led to substantial investigation to confirm its validity. Compared to other aspects of EF working memory has been relatively well researched in ID and as will be discussed below one of the reasons for this is interest in the poor auditory attention of individuals with Down syndrome which has been linked to their problems in developing language.

Salmon et al (1996) in a study investigating regional brain activity during a working memory task review the research into the Baddely and Hitch model. They note that some groups of patients have deficits in auditory short-term memory that can be attributed to either a selective impairment in the phonological store or to a disturbance to the articulatory process of rehearsal; other patients may have impairments to the central executive or visuo-spatial sketchpad. They observe that the neural basis of the phonological store appears to be the supramarginal gyrus of the left inferior parietal lobe and Broca's area for the articulatory rehearsal process. Visuo-spatial sketchpad deficits seem to be associated with a number of brain regions in both the right and left hemispheres; spatial and visual memory tasks lead to different patterns of activation. Salmon et al note that one of the difficulties in the 
Baddeley and Hitch model is distinguishing the role of the central executive form its two slave systems and in their study investigate one activity of the central executive - the updating process - which is believed to be independent of them. Utilising positron emission tomography (PET) they found that the updating process was associated with mid-dorsolateral prefrontal activation and which therefore was most probably responsible for the updating function of the central executive.

Petrides (1998) emphasises the role of the mid-dorsolateral cortex in monitoring and manipulating information held in working memory. Petrides describes how damage to this area of the brain in monkeys can lead to impairments in self-ordered memory tasks even though other aspects of memory are unaffected. Studies in humans carried out by Petrides using PET also suggest that this part of the brain is involved in selfordered memory tasks. In contrast to the monitoring and manipulation function of the dorsolateral cortex Petrides proposes that the mid-ventrolateral region is involved in the active but not automatic retrieval of information and cites a number of neuroimaging studies, which support this idea. Petrides also proposes that the ventrolateral cortex is activated when information is initially received from posterior association areas and when active comparisons of information held in working memory are made.

D’Esposito et al (1998) analysed a number of magnetic resonance imaging (MRI) studies of working memory which confirmed that the ventral areas of the prefrontal cortex are important in the maintenance of information and the dorsolateral cortex in the manipulation of information; however they suggest that hemispheric organisation rather than a dorsal/ventral sub division reflects the type of information that is held. They propose that the left hemisphere is more important in processing non-spatial and the right hemisphere spatial information.

A more recent review (Duncan and Owen 2000) and further neuroimaging research (Tsukira et al 2001) note the importance of the mid-ventrolateral, mid-dorsolateral and anterior cingulate cortex in working memory systems and confirm that working memory can be fractionated into maintenance and manipulation processes. 
Interestingly, because it is one of the working memory tasks investigated frequently in ID, Digit Span shows different types of activation in the prefrontal cortex (Owen et al 2000). Using PET Owen et al found that Digit Span Forward activated the mid-ventrolateral but not the mid-dorsolateral cortex whereas Digit Span Backwards activated both regions. This finding is consistent with the prediction that working memory is fractionated into two processes.

Studies of working memory in ID have focussed largely on the role of the phonological loop because of its relationship to language development (Gathercole et al 1992) but some studies have also considered the role of the visuo-spatial sketchpad and the central executive

Rosenquist et al (2003) investigated differences in the storage and rehearsal components of the phonological loop and visuo-spatial sketchpad in a group of individuals with ID matched with individuals without ID on a memory span task. Earlier studies (Hulme and Mackenzie 1992, Jarrold et al 2000 had suggested that individuals with ID may have inefficient or impaired ability to utilise sub vocal rehearsal processes rather than any problems with the capacity of the phonological store. Previous research however had not established if the difficulties in using rehearsal processes in the phonological loop also applied to the visuo-spatial sketchpad.

Rosenquist et al tested participants on a word length task, a phonological similarity task, visual complexity task and a visual similarity task. The authors' hypothesized that if the problems were related to rehearsal processes they would fine a reduced word length effect and also a reduced phonological similarity effect. They noted however that a reduced phonological similarity effect could also be related to a problem in the capacity of the phonological store. In contrast to participants without ID participants with ID showed no recall advantage for short over long words, which supported the suggestion that they were using inefficient rehearsal processes. Rosenquist et al also indicated that rather than using rehearsal processes individuals with ID may be relying on redintegration to recall words; this process involves the 
use of long-term phonological representations to fill in or reconstruct a decaying phonological memory. Another possible explanation offered was that individuals with ID are more likely to use visual or semantic coding. As the words used in the tasks were concrete and easily imageable then if visual or semantic coding was used word length would not be so relevant and could account for the almost equal number of short and long words recalled by the ID group.

In the phonological similarity task participants with and without ID recalled more dissimilar than similar words which indicated that the participants with ID were able to use the phonological store component of the system; the authors were unable to conclude however if there were capacity differences between the two groups. Interestingly the problems of rehearsal observed in the phonological loop component did not seem to apply to the visuo-spatial sketchpad. Indeed the individuals with ID performed as well as those without ID in the visuo-spatial sketchpad measures - the visual complexity and visual similarity tasks.

The authors concluded that their research supported earlier findings indicating that individuals with ID have problems in using sub-vocal rehearsal processes in the phonological loop but do not have difficulties in coding or storing information phonologically. They suggest that people with ID may use the apparently intact visuo-spatial sketchpad system to compensate for difficulties in using sub-vocal rehearsal. Henry and Maclean (2002) also found that although individuals with ID performed more poorly than a typically developing comparison group matched for mental age their performance was higher on most visual working memory tasks.

In their study Rosenquist et al were unable to arrive at any conclusions about the involvement of the central executive but noted that its function, i.e. to coordinate the phonological loop and visuo-spatial sketchpad could be implicated in rehearsal processes. They felt that this was an unlikely explanation in their study because if the central executive was involved it should also have affected rehearsal processes in the visuo-spatial sketchpad. The role of the central executive in working memory in individuals with ID has been investigated by the Forward Digit Span task, which is believed to be a measure of phonological loop capacity (Conners et al 1998). 
Conners et al suggest that because longer and longer digit strings are used the phonological loop resources become exhausted and the central executive is utilised. They compared a group of children with mild ID with two comparison groups, one of children matched for chronological age and one of children matched for verbal age on a Digit Span Forward task and found that highly significant group differences were reduced to non-significance when a measure of executive functioning was co varied out. Conners et al further suggest that Digit Span in ID keeps pace with verbal development but lags behind chronological age; this may indicate that the development of verbal abilities and executive function are associated. As discussed earlier the role of the central executive in Digit Span is supported by neuroimaging evidence in that the frontal lobes are activated by both Digit Span Forward and Backwards tasks (Owen et al 2000).

As previously mentioned the interest in the phonological loop component of working memory has been because of its association with language development and this is particularly salient for individuals with Down syndrome that not only have relatively poor verbal span in comparison with other individuals with ID (Vicari et al 1995) but also have relative difficulties in language development.

A number of studies (Jarrold et al 2000, Laws and Gunn 2004) have cautiously suggested that, taking into account the lack of sub-vocal rehearsal abilities in Down syndrome and ID generally, the hearing and articulation difficulties found in Down syndrome, the problem in Down syndrome may lie in the capacity or stability of information in the phonological store. This view has been partially supported by Vicari et al (2004) who have also suggested that it may be important to investigate the role of EF in working memory in Down syndrome.

\section{Measurement Issues in EF}

The difficulties in the measurement of EF have been well described by a number of researchers in this area (Denckla 1996, Pennington et al 1996, Burgess 1997, Phillips 1997, Wilson 1997, Hughes and Graham 2002) and can be briefly summarised as follows. 
The concept of EF rests upon the contrast between controlled actions to do with new situations and automatic processes which gradually come into play once the novelty of the task has been overcome. This can mean that it may be difficult to distinguish executive from non-executive tasks and can lead to problems of poor test reliability.

Another associated measurement problem in EF relates to the complex nature of many EF tasks and their impurity in regard to the involvement of more than one EF process as well as non-executive processes. Many EF tasks rely on adequate verbal and perceptual abilities so that deficits or conversely particular ability in a nonexecutive process may give a false impression of EF abilities in an individual. As an example of this Phillips (1997) notes that verbal fluency is generally regarded as a novel EF task because in most everyday situations searches are not carried out using phonemic criteria. Crossword experts however, may find verbal fluency a more familiar task and give a false impression of EF ability in this area. Similarly as Pennington et al (1996) have observed some (bright) individuals with no EF deficits will fail the Wisconsin Card Sort Test because they are testing out very complex sorting rules.

The measurement difficulties of EF are further compounded by the finding of cognitive congruence, which shows that in the undamaged brain performance on almost any cognitive task correlates positively with performance on another. This raises the question of whether EF can be separated entirely from the notion of " $g$ " or general intelligence. Burgess (1997) however presents evidence which indicates that performance on executive measures share a greater relationship with each other than can be explained by cognitive congruence or accounted for by the level of performance across a range of non specific tasks.

Related to the difficulties in task purity is the problem of low process-behaviour correspondence. A number of psychological processes are important in particular situations but not others, e.g. face recognition systems are activated when pictures of new faces but not new words are presented to a subject (Phillips 1997). Executive 
processes are used in a variety of situations and as outlined above may be affected by non-executive processes. As Wilson (1997) discusses:

"Standardised (EF) tests are poor at predicting real life performance because they do not take into account such factors as motivation, personality, individual styles, family support and previous reinforcement history (p240). Rarely, in neuropsychological tests are patients required to organise or plan their behaviour over longer time periods or to set priorities in the face of two or more competing tasks. Yet it is these sorts of executive abilities that are a large component of many everyday activities.”(p242).

Before discussing how these measurement issues particularly affect research of EF in ID it may be helpful to comment further on the relationship between EF and function of the pre-frontal lobes. As discussed earlier the frontal lobes have functions other than executive processes (Denckla 1996) and damage to areas outside the frontal lobes may also give rise to EF deficits (Robbins 1998). However as Phillips (1997) notes executive tests have mainly been validated to the criterion of frontal lobe sensitivity. Phillips suggests that what is more important is to consider construct validity, i.e. does an EF task measure what it claims to do?

In terms of criterion validity, i.e. the relationship of EF tasks to functioning in the pre-frontal cortex there is now ample evidence from neuro-imaging studies that EF tasks do activate this area of the brain as well as other brain regions (Petrides 1998, Robbins 1998, Duncan \& Owen 2000, Cazalis et al 2003).

Evidence that EF measures have construct validity is supported by studies which show that EF tasks correlate more closely together than to other cognitive measures (Burgess 1997, Robbins 1998). Support for construct validity also comes from evidence demonstrated by an investigation into the seperatability of three EF functions - mental set shifting (shifting), information updating and monitoring (updating) and inhibition of pre-potent responses (inhibition) (Miyake et al 2000). Miyake et al examined these functions in a set of frequently used executive tasks including the WCST. Factor analysis indicated three executive functions were 
moderately correlated with one another but were clearly separable. Structural equation modelling indicated that the three functions made differential contributions to performance on executive tasks.

One of the major assessment issues of EF in ID where brain damage is developmental and diffuse concerns purity of EF measures. This may be particularly salient for language abilities where comprehension could make it difficult for an individual to understand an EF task. Hughes and Graham (2002) note the important role of language itself in executive function and cite the work of Zelazo $(1999,2000)$ who suggests that language is important in guiding behaviour and in enabling experiences to be stored in working memory. The implication from this work is that developmental improvement in $\mathrm{EF}$ is associated with developmental increase in language ability and cannot be studied separately. An associated difficulty involves the already discussed issue of absence or impaired ability to use sub vocal rehearsal processes in verbal working memory, which may make it difficult for individuals with ID to verbally guide their way through EF tasks. The association of language with EF ability suggests that in investigations using ID participants it is particularly important to compare groups with similar language abilities.

A practical difficulty in measuring $\mathrm{EF}$ in ID is that most EF tests commercially available were standardised on adults with focal lesions and do not provide normative data for adults with ID where EF deficits are part of global impairment and have developmental trajectories.

One approach to assessing EF in ID is to use tasks which are developmentally appropriate and which have been simplified to reduce the confounding effects of non-executive problems interfering with the executive demands of the task; instead of comparing individuals with ID to typically developing adults it maybe more helpful to compare their performance to that seen in children at different developmental stages. This approach does not assume that the development of EF is identical in individuals with ID and typically developing children but at least gives some indication of comparative ability on EF tasks. This approach is particularly 
appropriate in PWS given the observations by Holland et al (2003) that the compulsive and other behaviours in PWS are related to arrested development.

\section{Development of EF Abilities in Children}

A number of studies support the view that the development of EF in children is a multi stage process with some functions developing at different rates. Barkley (1996) sets the scene by suggesting that the immature status of the PFC of the infant permits the development of the motor inhibition and delayed response patterns that serve as a foundation to the development of other executive functions. Working memory, forethought and a sense of time emerge next and overlap with the progressive development of inhibitory and delaying abilities. The internalisation of language emerges somewhat later and again overlaps with the ongoing development of earlier executive functions. Eslinger (1996) succinctly describes this process,

"Another way to conceptualise development of EF in relation to neurobiological dynamics is that differentiation of functions must precede and then synchronise with integration of functions" $\mathrm{p} 386$.

Gerstadt et al (1994) investigated inhibitory control in a group of 160 typically developing children aged between three and a half and seven years using a version of the Stroop task - the Day-Night test. In this task participants have to inhibit the response to say "day" when presented with a picture of the sun and say "night" instead and inhibit the response to say "night" when presented with a picture of the moon and stars but say "sun" instead. In a controlled version of the task participants learn to say night and day when presented with two different abstract designs. There were sixteen trials on both the experimental and controlled conditions. Children at age three and a half years were able to do the control version of the task successfully and there were significant improvements in performance on the experimental condition between the ages of three and a half and seven years. The mean percentage of correct response of children aged less than six years was less than $80 \%$ whereas children over six years were correct in approximately $90 \%$ of the trials. The authors 
concluded that these changes in performance were possibly due to improvements in inhibitory ability rather than changes in memory ability.

Levin et al (1991) studied developmental changes in EF in a group of fifty-two typically developing children split into three age groups - 7-8 years, 9-12 years and 13-15 years. The tests used included the Wisconsin Card Sort Test (WCST), Verbal Fluency, The Tower of London (TOL) and the Go-No Go task (Drewe1975), which is useful for measuring inhibition. Major gains were made on the WCST and the GoNo Go task by the age of 12 but the older 13-15 year age group made further advances in performance in the TOL. The authors noted that a weakness of their study was that it was cross sectional and had a relatively small sample size, however they stated that their findings were consistent with previous research.

The development of planning ability as measured by improved performance in the TOL has also been investigated by Anderson et al (1996) using a large group of 376 children aged between 7 years and 13 years 11 months. They found the greatest increments in performance between the ages of 7 and 9 years and again between 11 and 12 years. Taking into account previous research the authors noted that the incremental changes seen at ages 7-8 years represent a developmental "spurt" related to increases in both the speed of processing and planning ability; they related changes at age 11 years to a second "spurt" but this time associated with only improvements in processing speed. A further developmental "spurt" at age 12 was however associated with significant increases in planning and problem solving ability possibly reflecting maturity of the underlying neural systems.

Recently Luciana (2003) has described studies carried out on the development of EF using the Cambridge Neuropsychological Testing Automated Battery (CANTAB). The CANTAB, as has been previously discussed, was developed for investigating EF in adults and consists of automated tests, which measure working memory planning, visual memory and visual attention. Luciana's rationale for using the CANTAB with children is that although simplified EF tasks (for children) may tap the PFC they are not so useful for determining when adult levels of proficiency are 
reached. Luciana has also indicated concerns that much previous research in this area has relied on cohorts of children representing distinct age groups and was not longitudinal in nature. Luciana's studies have investigated EF abilities using the CANTAB in children aged from 3 years through to adulthood. Consistent with earlier research, Luciana has found that planning and working memory skills have not reached adult levels by the age of 12 years. Luciana suggests:

"that the functional maturity of prefrontal circuitry at a level that would permit simultaneous processing of multiple levels of information, is not reached until post puberty coincident with the elimination of pruning back of synapses in the developing neocortex (p172).....myelination not extensively discussed here is another candidate process that could serve to enhance synaptic activity"(p173).

Whilst the approach of Luciana and Levin in using adult EF tasks is useful for indicating when adult levels of performance are reached it is important to recall one of the problems of EF tasks is that they may measure more than one EF function. Over the course of development the functions utilised within one EF task may vary. To illustrate this problem Bull et al (2004) in a study comparing two related planning tasks, The Tower of Hanoi (TOH) and the Tower of London, note that whilst in adults visuo-spatial working memory may be important in visually planning moves ahead, this may be less likely in young children who do not appear to overtly preplan their moves before implementation. Bull et al suggest that younger children may rely more on a perceptual strategy of deciding the move sequence on line. In their study Bull et al found that the role of inhibition was more prominent for the TOL by young children aged 4 years 8 months and that shifting or mental flexibility was more important in the $\mathrm{TOH}$.

Physiological and neuroimaging evidence concerning the development of the frontal lobes that are associated with EF also supports the idea that this process takes place in stages. Thatcher (1991) in a paper considering studies that have investigated frontal lobe development by EEG coherence measures in children suggests that the establishment of corticocortical connections with different regions of the frontal 
lobes occur sequentially and dominates post-natal cerebral development from birth to 16 years. Periods of rapid growth occur within five different age ranges and they are birth to 5 years, 7 to 9 years, 11 to 13 years, 14 to 16 years and 18 to 20 years. Thatcher has also observed that connections develop at different rates in the left and right hemispheres until approximately puberty (11 to 14 years). Paus et al (1999) measured the structured maturation of white matter in corticocortical and frontostriatal pathways in a large group of children and adolescents and found that the pathways related to speech and motor function in the left hemisphere were not mature until late adolescence.

Tamm et al (2002) have investigated developmental change in one executive function -inhibition using functional magnetic resonance imaging (fMRI) in a group of 19 typically developing participants ranging in age from 8 to 20 years. The task that they employed to measure inhibition was the Go- No Go task. No relationship between accuracy and age was noted but the ability to inhibit responses more quickly significantly improved with age. A further interesting finding of this study was that during the task younger participants activated discrete regions of the PFC more extensively than older subjects. Older subjects showed more focal activation, particularly in the left inferior frontal gyrus, orbitofrontal gyrus and the adjoining insula. Tamm et al suggested that although younger subjects performed the task adequately it was possible that they used less efficient strategies that required recruitment of more widespread regions of the dorsolateral cortex and a number of executive functions in addition to inhibition including working memory and set shifting.

The authors felt the activation of the orbitofrontal cortex in older subjects may be related to its role in controlling voluntary goal directed behaviour, strategic memory and supporting the mobilisation of behavioural strategies for cognitive tasks. An important implication of the paper was that conclusions regarding the functional roles of the PFC in adults might not be directly applicable to children. 
DeBellis et al (2001) have utilised neuroimaging techniques to investigate gender differences in brain maturation during childhood and adolescents in a group of 118 typically developing children and adolescents aged between 6 to 17 years. Significant age related decreases in cerebral grey matter and increases in cerebral white matter volumes and corpus callosal areas were found in the overall sample. However they found different rates of development with males showing more prominent age related grey matter decreases and white matter volume in corpus callosal area increases compared with females. The authors noted that white matter increases could be due to myelination, increases in axonal size, glial proliferation or a combination of these. Grey matter decreases, on the other hand, probably reflect dendritic pruning processes. The authors observe that sex steroids play an important part in neurodevelopment, estradial influences synaptogenesis and delays synaptic pruning; testosterone may be associated with myelinogenesis. They point out that hormonally influenced patterns of brain maturation may be of significance in the aetiology of psychiatric disorders, e.g. schizophrenia which may be mediated by the excess elimination of synapses.

As discussed above age and gender are both important factors in the maturation of the brain and in respect of EF it is likely that in different stages of development EF tasks may utilise different functions. Thus although a developmental approach may be helpful in investigating EF in adults with ID caution must be shown in assuming that because individuals with ID are performing at a similar level in an EF task to typically developing children the same functions are being used and the same areas of the PFC being recruited. Numminen et al (2001) for example, investigated performance in the $\mathrm{TOH}$ in adults with ID and found that their performance was equal to a group of typically developing children matched for fluid intelligence. However in the ID group visuo-spatial but not phonological working memory abilities were connected to performance and that poor performance was associated with poor inhibitory abilities. 
Aims and Hypotheses of the Present Study.

The aim of the present study is to explore the possibility that relative deficits in EF abilities may underlie the relatively high rates of maladaptive behaviour reported in PWS.

It is predicted that,

- In comparison with a group of individuals matched for verbal ability, a group of individuals with PWS will show significantly higher rates of maladaptive behaviour

- The group of individuals with PWS will show higher rates of behaviour indicating executive dysfunction.

- The group of individuals with PWS will show significantly poorer ability on a range of EF tasks

- Poorer performance on EF tasks for both groups will be positively correlated with ratings of maladaptive behaviour or behaviour indicating executive dysfunction

- Performance on EF tasks for both groups will be positively correlated with verbal abilities.

Differences in EF performance between the two sub types of PWS will also be explored but no predictions are made about the direction of any differences. 


\section{$\underline{\text { Materials and Methods }}$}

\section{Experimental Design}

As is explicit in the aims of the study it was decided to use a between subjects rather than within subjects design. Primarily this design was adopted because the author's reading of the literature pointed to the strong possibility that there may be relative $\mathrm{EF}$ deficits in PWS compared to other ID groups, but there were a number of additional reasons for making this decision. One reason related to the paucity of data available regarding the performance of participants with ID on either EF or non- EF cognitive tests. Many EF and non-EF cognitive tests are standardised only on typically developing populations. Without the opportunity to make comparisons using appropriately standardised data it was possible that erroneous conclusions regarding relative ability at a particular aspect of EF or non-EF cognitive ability could be drawn. By using a between subjects design this problem could be avoided. A further related reason for using a between subjects design related to the suggestion of Garner et al (1999) that EF deficits may be a general feature of ID. If this were the case for individuals with PWS then one might have expected to find deficits in EF relative to other types of cognitive ability, however unless comparison of EF ability was made with another ID group it is difficult to establish how salient this might be for individuals with PWS.

\section{$\underline{\text { Participants }}$}

Nineteen adults with a clinical diagnosis of PWS were recruited from a multidisciplinary PWS clinic, organised by a consultant paediatric endocrinologist, at a national children's hospital, with a special interest in PWS. Although the clinic is organised by a paediatrician any individual with PWS regardless of age residing in Scotland can be referred to it. The main aim of the clinic is to support good physical and mental health in affected individuals and to give advice to parents and carers. Referral to the clinic is usually made by a GP.It is important to note that other than having a definite or probable diagnosis of PWS no specific referral criteria e.g. 
obesity or behaviour problems are required. Multi-disciplinary input currently includes medicine, psychiatry, dietetics, speech and language therapy, and nursing. Nineteen is a relatively small sample size, however it represents between $25-30 \%$ of the adult PWS population in Scotland, which based on Whittington et al (2001) prevalence figures is between $60-80$ individuals.

In order to control for developmental changes in brain structure, only individuals over the age of 16 were recruited into the study. Eleven of the participants were male and eight were female. All the participants had appropriate genetic testing carried out by a molecular geneticist, and all but one of the participants, a female, was found to have a genetic abnormality associated with PWS. This female has been included in the study because the consultant paediatrician was certain that she has PWS. She has the clinical features of PWS and there is a possibility that she has a micro-deletion on chromosome 15 , which is undetectable using currently available molecular genetic testing techniques. This female was not included in any of the analyses where a comparison of the deletion and UPD subtypes was made. Six individuals, five males and one female, were found to have UPD, and twelve individuals, six males and six females, were found to have deletions.

The age range of the PWS participants was between 16 and 49. As language abilities appear to be related to the ability to do EF tasks (Hughes \& Graham 2002), it was decided to control for verbal IQ which was estimated using the vocabulary and similarities sub-tests of the Wechsler Adult Intelligence Scale - Revised (WAIS-R, Wechsler 1981). Whilst this does not equate to the verbal IQ score obtained by using all the Wechsler sub-tests, it does give a useful measure of language ability and is not confounded by attentional factors which may affect performance on some of the other verbal Wechsler sub-tests, eg digit span and arithmetic. Using this short form measure of estimating verbal IQ, the verbal IQ of participants ranged from 53 to 93 . The comparison group were also drawn from a clinical setting and consisted of fifteen individuals, seven males and eight females, of similar ages and verbal IQs to the PWS participant group. All the members of the comparison group had been seen as patients in the Psychology Department in which the author was employed, and had 
been referred to Clinical Psychology because of anxiety problems or difficulties in social interaction; apart from one female with Aspergers Syndrome, none had a diagnosis of Autistic Spectrum Disorder. One member of the comparison group had a genetic syndrome - Down syndrome - the cause of intellectual disability for the other participants with ID was not known.

One of the major difficulties in research on behavioural phenotypes is the choice of an appropriate comparison group. Comparison groups comprising children of similar developmental level, individuals with Down syndrome (because it is the most common genetic syndrome), or individuals with mixed aetiologies of ID but similar levels of ability are often used. Sometimes a comparison group may comprise typically developing individuals who have a similar behaviour problem. Hodapp and Dykens (2001), who have carried out substantial research on several behavioural phenotypes, describe some of the strengths and weaknesses of these different approaches; they suggest that if a comparison group comprises individuals of similar age and ability the aetiologies for ID should be well and truly mixed rather than include only indiduals with non-specific ID as is often the case. In the present study the reason for using a comparison group with similar levels of verbal ability has already been described. Prior to the development of the study the author was told by a number of parents of adults with PWS that their sons/daughters would be very anxious about the proposed testing which is why it was decided to use a comparison group who might also be anxious in a novel test situation. As there is already some data on EF in children available in the literature, and also because of the practical and ethical difficulties involved in recruiting children into a pilot study of this kind it was decided not to include an additional comparison group of developmentally similar children to the PWS adults at this stage.

The age range of the comparison group was between 18 and 49, and the range of estimated verbal IQ was between 55 and 91. Demographic details of the PWS participant and comparison groups are shown in table 1. There were no significant differences in mean verbal IQ and age between the two groups, as measured by independent $\mathrm{t}$ tests (age, $\mathrm{t}=0.379$, sig $=0.71 ; \mathrm{IQ}, \mathrm{t}=0.667$, sig $=0.51$, both tests two- 
tailed). Nor were there significant differences in the gender distribution of the two groups measured by the chi-squared test for independence (chi-squared $=2.42, \mathrm{df}=1$ ,sig $>0.05$,two-tailed).

\section{TABLE 1}

Descriptive characteristics of the PWS and comparison groups

\begin{tabular}{|l|c|c|c|c|c|}
\hline & $\mathbf{n}$ & mean age (SD) & male & female & 8 \\
\hline PWS & 19 & $23.5(7.6)$ & 11 & $72.8 \quad(10.5)$ \\
\hline Comparison & 15 & $24.5(8.7)$ & 7 & 8 & $70.6(8.6)$ \\
\hline
\end{tabular}

\section{Ethical Approval}

Ethical approval for the study was obtained from the Hospital Trust in which the multi-disciplinary PWS clinic is based, and for the purpose of recruiting the comparison group, also from the author's employing Trust. Each participant was sent an information letter and asked to sign a consent form. The participants were all adults but parents/carers were also supplied with an information letter and where appropriate signed the consent form on behalf of their son/daughter. The participants' GPs were also sent an information letter about the research and were invited to comment if they had any concerns. Examples of these letters and forms with identifying information removed are contained in appendix A.

Testing was carried out by the author at a location of the participants' choice, this was usually either their home, or for some of the participants with PWS, a quiet room at the hospital where the PWS clinic was held.

\section{Measures}

One of the difficulties in assessing executive function (EF) is that there is no such thing as a pure measure of EF; tasks that are believed to tap EF executive function also utilise other cognitive abilities (Burgess, 1997; Hughes and Graham, 2002). 
In participant groups that include individuals with ID, it is probable that general cognitive ability could affect performance on EF tasks. For this reason, a set of measures was chosen which had either been used previously in studies involving individuals with $\mathrm{ID}$, or had been drawn from the literature on developmental psychology. The intention was to keep potentially confounding effects of comprehension difficulties to a minimum.

The measures chosen investigated abilities which are believed to be important in studies of EF, ie initiation of behaviour, planning, set/rule changing, the inhibition of a pre-potent response, and working memory. An important feature of an EF task is that it should be novel and as far as could be ascertained all the EF tasks were novel to the PWS and comparison groups.

\section{Initiating Behaviour}

Verbal Fluency

Two variations of this well-known and established neuropsychological task were given to participants. The first was a traditional verbal fluency FAS task (Newcombe 1969); participants were asked to produce as many words as they could beginning with the letters F, A and S.One minute was allowed for each letter. Participants were asked not to use the names of countries or Christian names; words with different endings but the same stem were only scored once. Participants were given a score for the number of words produced for each letter and from that a total score was derived. The number of errors, and number of words that were repeated were also recorded. In order to allow for the possibility that some individuals with poor verbal abilities would have difficulty with this task, a different version was also given to every participant; participants were asked to name as many items as possible from three semantic categories, a minute was allowed for each category; the categories were animals, objects and occupations. This version of the test has been used by Temple et al (1996) in a study of EF in children with Turners Syndrome.

Verbal fluency is widely recognised as an $\mathrm{EF}$ task because it requires the generation of novel responses; this view has however been challenged by Phillips (1997) who has found that verbal fluency ability is related to age and intelligence and suggests 
that deficits in verbal fluency should not automatically be assumed to be caused by executive dysfunction.

\section{Planning Ability}

\section{Tower of London (TOL)}

A standardised concrete adaptation of the TOL task (Shallice 1982) was used to assess planning ability, the TOL Drexel version (Culbertson and Zilmer 1998a). The TOL Drexel version is reported to have good construct validity (Culbertson and Zilmer 1998b).

This version of the TOL provides detailed instructions for the administration of the task, which were followed in the current study. The TOL task comprises a board on which there are three vertical pegs placed equally apart. The pegs are of different sizes, on which can be placed, in the case of the largest peg, three coloured blocks green, red and blue, with holes through them. The other two pegs can take two blocks and one block respectively. The task required the participants to move the blocks from an initial starting position which is constant for all problems, to an end position illustrated on a sheet of paper, one for each problem. To do the task successfully, participants had to move the blocks in a minimum number of moves; problems increased in difficulty from those which could be done in a minimum of three moves to problems which could only be done in a minimum of seven moves. Each level of move had three different problems, giving a total of fifteen problems altogether. Participants had to follow three rules in attempting the task, only one block could be picked up at a time, and once a block had been picked up it had to be placed on to a peg and could not be put down elsewhere whilst another block was moved. Participants were also not allowed to put more blocks on the pegs than there was space for. Participants were given three practice two-move problems, in order to help them understand what they had to do in the task, and to ensure that they were aware of the rules.

Participants were given up to two minutes to solve each problem. The number of problems correctly solved in the minimum number of moves within the allotted time 
limit provided a score. In some Tower studies planning time is recorded, however Phillips et al (1999) have found that this variable does not relate to the accuracy of performance on the task and was not measured in the present study.

The Drexel version of the TOL was used because it provides clear administration instructions, and also because it includes more difficult six and seven move problems. Some EF studies e.g. Temple (1996) have used a version of the TOL, which only includes up to five move problems. A further advantage of the Drexel version of the TOL is that it allows for up to two minutes to complete each of the problems. Tower tests have been criticised because they put participants under too much time pressure, which may not accurately reflect EF abilities (Bishop et al, 2001).

In order to control for difficulties in visuo-spatial ability, participants were also given the Block Design sub-test from the Wechsler Adult Intelligence Scale - Revised (WAIS-R).

\section{Set/ Rule Changing Ability and Inhibition of a Pre-potent Response}

A number of tests were given which are believed to have some validity in assessing these abilities, and which were accessible to a range of ability levels.

\section{Luria Hand Game Adapted by Hughes (1996)}

This test was adapted from Luria's original hand game, to investigate $\mathrm{EF}$ in children with Autistic Spectrum Disorder (Hughes 1996). The administration of the task followed that given by Hughes in her paper. Participants were taught a simple game of motor inhibition. They were first taught to model two hand-gestures, a pointed finger and a clenched fist, and were then required to produce the opposite gesture shown by the tester, that is if the tester produced a clenched fist, the participant was expected to demonstrate a pointed finger. Participants had to inhibit the natural response to imitate the tester. Each participant was given fifteen trials. 
$\underline{\text { Rule Shift Card Test - Wilson et al (1996) }}$

This test, which is part of the Behavioural Assessment of Dysexecutive Syndrome (BADS Wilson et al 1996) used 21 spiral bound, non-picture playing cards, and investigated participants' ability to respond correctly to a rule and then to shift from that rule to another one. The administration of this task followed the instructions given by the authors.

In the first part of the task participants were asked to say 'yes' when they saw a red card, and 'no' when they saw a black card. This rule was printed on a card which was left in full view throughout the test to reduce memory constraints. In the second part of the test participants were asked to respond 'yes' if the card that had just been turned over was the same colour as the previously turned card, and 'no' if it was a different colour. The challenge in the second part of the task was therefore to concentrate on applying the new rule. As in the first part of the task, the new rule was left in full view of the participant. If a participant was unable to read the rules, the tester read them until the participant was able to recall what the rule was. Recall of the rule was checked at the end of each part of the test. The number of correct responses was recorded.

\section{Spatial Reversal Task - McEvoy et al (1993)}

This task was also developed to investigate EF in children with Autistic Spectrum Disorder, and the procedure used in the current study followed the instructions for administration provided by the authors (McEvoy et al 1993).

Participants were asked to search for an item (a coin), which was hidden under one of two cups, the cups were initially screened from the participant so they were not aware where the coin had been placed. After each search the screen was put back but the position of the coin was not changed until the participant was able to find it on four consecutive occasions. Its position was then changed to under the other cup and the process repeated. A total of twenty-five trials in total was given, and the number of set changes (ie runs of four) successfully completed was recorded. 
Stroop Type Task - Day/Night Version - Gerstadt et al (1994)

As the adult version of the Stroop Test requires fluent reading ability, the day/night Stroop Task (Gerstadt et al, 1994) was used; this task was developed to assess inhibition in 4-7 year old children who may not have fluent reading abilities. Gerstadt et al, give instructions for administering the task and these were followed in the current study.

In this task, participants were asked to say 'night' when they saw a picture of the sun drawn on a white card, and 'day' when they saw a picture of moon and stars drawn on a black card. After being given a two card practice trial, participants were then shown a sequence of sixteen cards arranged in a set order. Participants were also given a control version of the task in which they were taught to associate two separate abstract drawings with the words 'night' and 'day' and then again after a short practice given a trial of sixteen cards in an arranged order. The time taken and the number of trials where the correct response was given was recorded.

\section{Working Memory}

Self-Ordered Pointing Test - Temple et al (1996)

In this task, which is an adaptation of a self-ordered pointing task devised by Petrides and Milner (1982), participants using their own strategies, had to remember the sequence in which they pointed to a set of visually displayed stimuli. The task has two versions both of which were given to participants, one uses black and white abstract designs and the other representational (concrete) black and white drawings of everyday objects. Each version of the test comprises sets of six, eight, ten and twelve pages. On each page there are an equivalent number of designs or drawings. The designs/drawings in each set are the same but are in different spatial positions on each page. The participants were asked to point to a different design/drawing each time a page was turned. Scores were based on the number of pages correctly completed before an error was made, the number of times the same item was pointed to in a set (item perseveration), and the number of repeated points made to the same spatial position (position perseveration). Overall total scores were obtained, as well as separate scores for each set of stimuli. 


\section{Digit span from the WAIS R (Wechsler 1981)}

As auditory processing problems are reported in PWS, participants were given this sub-test from the WAIS R, which is in two parts. Digits Forward is a useful measure of auditory attention and requires participants to recall strings of digits, which increase in length by one after every second presentation. In Digits Backward, participants have to recall similarly increasing strings of digits in reverse order. Digits Backwards is often thought of as being a working memory task. The maximum number of digits separately recalled forward and backwards was recorded, as was the difference between these two figures.

\section{Behaviour}

In addition to the $\mathrm{EF}$ tasks given to participants, the following questionnaires were given either to a carer, or a member of staff who knew the participant well.

\section{Dysexecutive Questionnaire (DEX)- Wilson et al (1996)}

This questionnaire is part of the Behavioural Assessment of Dysexecutive Syndrome (BADS), Wilson et al (1996) and consists of 20 items constructed to sample a range of difficulties commonly associated with dysexecutive problems, it covers four areas - emotion and personality, motivation, behaviour and cognitive function. Each item is rated on a five point scale representing problem severity. The total score for the questionnaire was recorded. This questionnaire was used because of its focus on EF; it has not been previously used on an ID population.

Aberrant Behaviour Checklist (ABC), community version, Aman et al (1985a) and Marshburn and Aman (1992), Aman and Singh (1994).

This is a fifty-eight item questionnaire, which requires a rating of specified maladaptive behaviour from zero to three representing problem severity over the previous four weeks. As well as providing a total score, the $\mathrm{ABC}$ yields scores from five factors, (1) irritability and agitation (2) lethargy and withdrawal (3) stereotypic behaviour (4) hyperactivity and non-compliance (5) inappropriate speech. The ABC was used in addition to the DEX because of its reliability in assessing behaviour 
problems in people with cognitive impairment, and because it has been used in a previous study in PWS by Clarke et al (1996). The psychometric characteristics of the $\mathrm{ABC}$ are reported in Aman et al (1985b) and Aman et al (1995).

\section{Experimental Design and procedure}

The investigation was a between subjects design, in which the PWS and comparison group participants were tested on the EF and other tasks described above. An informant, usually a parent or carer, completed the behaviour questionnaires for each participant. The testing took about one and a half hours to complete. As the experimental design was between subjects the EF tasks were given in the same order to all participants, however in order to avoid practice/familiarity effects between similar types of tasks they were given in the following order, Verbal fluency, Spatial Reversal, TOL, Stroop-type task, Self-ordered pointing task, Luria hand game, Vocabulary, Rule Shift Card test, Block Design, Similarities, and Digit Span.

\section{Exploratory Data Analysis}

Before statistical analyses were performed the data was entered onto SSPS Version

12 spreadsheets and double-checked for accuracy. The SSPS explore data facility was used to plot out the distribution of the data. Visual inspection of the data showed that the data appeared to be normally distributed and accordingly it was decided that the use of parametric statistical procedures would be appropriate.

\section{Analysis}

The PWS and comparison groups were compared with respect to age and verbal IQ, and differences in performance on the EF tests and measures of behaviour (independent $\mathrm{t}$ tests). Correlations of EF performance with behaviour and verbal IQ were also investigated within the two groups (Pearson's test). Differences in performance (independent t tests) on and correlations (Pearson's test) between EF tasks and behavioural measures were also investigated for the two genetic sub-types, i.e. deletion and UPD, of the PWS group. As it was hypothesised that the PWS group and separately the two sub-types would perform more poorly than the comparison group on EF tasks, and that poorer performance on EF tasks would correlate with 
higher rates of maladaptive behaviour, but better performance with higher verbal IQ these data are reported at a one tailed-level. Significant results that were contrary to these predictions were however reported at a two-tailed level.

As no predictions were made concerning differences on $\mathrm{EF}$ performance between the deletion and UPD sub-types of the PWS group on their own, this set of data was reported at a two-tailed level. All the analyses were carried out using SSPS.

\section{Experimental Power}

Reference to power tables in Clark-Carter (1997) suggests that to obtain a medium effect size $(\mathrm{d}=0.5)$ using the sample size available, the power is 0.41 (one-tailed independent $\mathrm{t}$ tests). The power for the comparison between the deletion group and the comparison group was $0.31(\mathrm{~d}=0.5)$. This indicates that there is a high probability of Type II errors occurring, ie that there may be differences in performance and behaviour between the PWS and comparison groups but they may not be observed because of the low experimental power. To obtain power of 0.8 with a medium effect size $(d=0.5)$ it would have been necessary to have 50 participants in both the PWS and comparison groups.

\section{$\underline{\text { Results }}$}

\section{Differences in behaviour}

Mean scores and levels of significance (independent $t$ tests) are shown for the Aberrant Behaviour Checklist (ABC) and Dysexecutive Questionnaire (DEX) in Table 2. The PWS group had a significantly higher total ABC score than the comparison group $(\mathrm{p}<.05)$ and also showed significantly higher scores $(\mathrm{p}<.05)$ in factors 3 and 5, stereotypic behaviour and inappropriate speech. The PWS group had a higher group score on the DEX, but it did not reach significance. In both the PWS and the comparison group, the $\mathrm{ABC}$ total score and the $\mathrm{DEX}$ were positively correlated $(\mathrm{p}<.01)$. There were no significant correlations between verbal IQ and the scores on the ABC and DEX for either the PWS or the comparison group. Table 3 shows data for the total $\mathrm{ABC}$ and $\mathrm{DEX}$ scores for the deletion $(\mathrm{n}=12)$ and disomy 
$(n=6)$ groups. One participant was excluded from this and subsequent analyses because of uncertainty about the presence of a deletion. In this study the UPD subgroup had higher mean levels of behaviour problems but these differences were not significant.

\section{Table 2}

Differences between mean scores on the behavioural questionnaires for the PWS and comparison group (independent $t$ tests, one-tailed)

\begin{tabular}{|l|c|c|l|l|}
\hline $\begin{array}{l}\text { Aberrant Behaviour } \\
\text { Checklist (ABC) }\end{array}$ & & & & \\
\hline Mean (sd) & PWS (n=19) & Comparison(n=15) & $\mathrm{t}$ & sig \\
\hline Factor 1. Irritability/ agitation. & $15.16(9.66)$ & $9.79(9.34)$ & 1.601 & 0.06 \\
\hline Factor 2. Lethargy. & $12.68(9.80)$ & $7.86(6.70)$ & 1.588 & 0.06 \\
\hline Factor 3. Stereotypic behaviour. & $4.32(5.03)$ & $1.79(2.99)$ & 1.801 & $0.04 *$ \\
\hline Factor 4. Hyperactivity. & $6.11(7.34)$ & $8.00(8.16)$ & 0.701 & 0.25 \\
\hline Factor 5. Inappropriate speech. & $5.05(3.44)$ & $2.43(2.59)$ & 2.393 & $0.01^{* *}$ \\
\hline Total & $49.74(30.86)$ & $29.86(25.10)$ & 1.975 & $0.02^{*}$ \\
\hline & & & & \\
\hline $\begin{array}{l}\text { Dysexecutive Questionnaire } \\
(\text { DEX) }\end{array}$ & & & & \\
\hline Mean Total (sd) & $43.42(17.61)$ & $38.43(14.87)$ & 0.858 & 0.19 \\
\hline$*=\mathrm{p}<0.05 * *=\mathrm{p}<0.01$ & & & & \\
\hline
\end{tabular}

\section{$\underline{\text { Table } 3}$}

Differences between means of behavioural questionnaires and verbal IQ for deletion and UPD groups (independent $t$ tests, two tailed)

NB one participant was excluded from this analysis because of uncertainty about the presence of a deletion.

\begin{tabular}{|l|l|l|l|l|}
\hline Measure & Deletion $(\mathrm{n}=12)(\mathrm{sd})$ & $\mathrm{UPD}(\mathrm{n}=6)(\mathrm{sd})$ & $\mathrm{t}$ & $\mathrm{sig}$ \\
\hline ABC Total & $46.82(35.97)$ & $55.50(25.64)$ & 0.520 & 0.61 \\
\hline DEX Total & $43.09(19.01)$ & $50.00(14.56)$ & 0.771 & 0.45 \\
\hline Verbal IQ & $71.64(12.08)$ & $76.50(8.64)$ & 0.867 & 0.40 \\
\hline
\end{tabular}




\section{Differences in performance on EF tasks}

Table 4 shows the differences between the mean scores on EF tasks for the whole PWS and comparison group. Significant differences were only found in three tasks, the TOL, the Self-ordered pointing test and Digit Span.

The PWS group had significantly better performance $(\mathrm{p}<.05$, reported as two-tailed) on the 5 move and total correct problems made in the minimum number of moves on the TOL.There was also a trend towards significance for better performance by the PWS group on the ability to solve more complex TOL problems correctly. There were no significant differences in the Block Design task.

In the Self-ordered pointing task the PWS group made significantly more abstract position perseverations $(\mathrm{p}<.05)$, but were otherwise very similar to the comparison group.

The performance of the PWS group on Digit Span forwards was significantly poorer than the comparison group $(\mathrm{p}<.01)$, but the PWS group showed significantly less difference between Digits Forwards and Backwards $(\mathrm{p}<.05$, reported as two-tailed). 


\section{Table 4}

Differences between mean scores on EF tasks for the PWS and comparison groups (independent $t$ tests), (a) denotes significant or almost significant results which are contrary to hypothesis and which are reported as two-tailed, otherwise all results are onetailed.

\begin{tabular}{|c|c|c|c|c|}
\hline Measures & PWS(n=19)(sd) & Comparison $(\mathrm{n}=15)(\mathrm{sd})$ & $t$ & sig \\
\hline \multicolumn{5}{|l|}{ Initiating behaviour } \\
\hline \multicolumn{5}{|l|}{ Verbal Fluency FAS } \\
\hline F & $3.8(3.1)$ & $5.7(4.8)$ & 1.415 & 0.08 \\
\hline A & $2.7(2.8)$ & $3.6(4.0)$ & 0.793 & 0.22 \\
\hline $\mathrm{S}$ & $4.9(3.5)$ & $5.1(4.5)$ & 0.172 & 0.43 \\
\hline Total FAS & $11.4(8.8)$ & $14.2(12.3)$ & 0.785 & 0.22 \\
\hline Repeated words & $0.26(0.65)$ & $0.2(0.56)$ & 0.298 & 0.38 \\
\hline Errors & $0.11(0.32)$ & $0.07(0.26)$ & 0.383 & 0.35 \\
\hline \multicolumn{5}{|l|}{ Categories } \\
\hline Animals & $10.8(4.7)$ & $11.2(5.1)$ & 0.244 & 0.40 \\
\hline Objects & $10.8(5.9)$ & $11.1 \quad(5.6)$ & 0.139 & 0.45 \\
\hline Occupations & $7.8(4.1)$ & $8.3 \quad(3.6)$ & 0.367 & 0.36 \\
\hline Categories Total & $28.4(11.3)$ & $30.6(12.7)$ & 0.541 & 0.30 \\
\hline Category repeats & $0.65(1.01)$ & $0.4 \quad(0.91)$ & 0.692 & 0.25 \\
\hline Category errors & $0.0(0.0)$ & $0.13(0.52)$ & 1.000 & 0.17 \\
\hline \multicolumn{5}{|l|}{ Planning Ability } \\
\hline \multicolumn{5}{|l|}{ Tower of London TOL } \\
\hline \multicolumn{5}{|l|}{ TOL number of problems solved correctly in min. no of moves } \\
\hline 3 & $1.95(0.85)$ & $1.60(1.06)$ & 1.065 & 0.15 \\
\hline 4 & $1.84(0.96)$ & $1.40(0.83)$ & 1.417 & 0.08 \\
\hline 5 & $1.05(0.78)$ & $0.53(0.64)$ & 2.082 & $0.04 a^{*}$ \\
\hline 6 & $0.21(0.42)$ & $0.07(0.26)$ & 1.230 & 0.11 \\
\hline 7 & $0.21(0.54)$ & $0.20(0.56)$ & 0.056 & 0.47 \\
\hline Total & $5.26(1.67)$ & $3.80(2.31)$ & 2.151 & $0.04 a^{*}$ \\
\hline $\begin{array}{l}\text { TOL most complex problems solved correctly in min no of } \\
\text { moves }\end{array}$ & $5.21(0.98)$ & $4.2(1.98)$ & 1.956 & $0.06 \mathrm{a}$ \\
\hline \multicolumn{5}{|l|}{ Block Design } \\
\hline Raw Scores & $11.37(9.36)$ & $12.93(9.50)$ & 0.481 & 0.32 \\
\hline Age Scale Scores & $5.05(2.35)$ & $5.6(2.35)$ & 0.712 & 0.24 \\
\hline \multicolumn{5}{|l|}{ Set (Rule) Changing Ability and Inhibition } \\
\hline Luria Hand Game no of correct responses & $13.63(2.14)$ & $14.0(1.93)$ & 0.520 & 0.30 \\
\hline Rule Shift Card Test no of errors on rule 2 & $5.0 \quad(3.6)$ & $5.1 \quad(3.8)$ & 0.052 & 0.48 \\
\hline Spatial Reversal Task no of set (runs of 4 correct responses) & $2.84(1.17)$ & $2.47(1.55)$ & 0.806 & 0.21 \\
\hline \multicolumn{5}{|l|}{ Stroop - Day/Night Test } \\
\hline No. of errors in experimental condition & $1.79(3.94)$ & $2.20(3.65)$ & 0.312 & 0.38 \\
\hline No. of errors in control condition & $0.95(1.84)$ & $3.00(4.54)$ & 1.649 & 0.06 \\
\hline Time on experimental condition in secs. & $49.6(11.98)$ & $51.3(19.19)$ & 0.304 & 0.38 \\
\hline Time on control condition in secs. & $44.9(8.98)$ & $42.1 \quad(11.50)$ & 0.802 & 0.21 \\
\hline
\end{tabular}




\begin{tabular}{|l|l|l|l|l|}
\hline & & & & \\
\hline Table 4 continued & & & & \\
\hline Measures & PWS(n=19)(sd) & Comparison(n=15)(sd) & $\mathrm{t}$ & sig \\
\hline Working memory & & & & \\
\hline Self-ordered pointing test & & & & \\
\hline Total concrete pages before error & $30.68(3.35)$ & $30.40(5.17)$ & 0.194 & 0.42 \\
\hline Total abstract pages before error & $18.53(3.26)$ & $17.93(4.10)$ & 0.471 & 0.32 \\
\hline Total concrete position perseverations & $5.58(3.47)$ & $4.00(2.17)$ & 1.538 & 0.07 \\
\hline Total abstract position perseverations & $4.74(2.88)$ & $2.93(2.22)$ & 1.918 & $0.03 *$ \\
\hline Total concrete item perseverations & $2.63(1.34)$ & $2.40(2.56)$ & 0.341 & 0.37 \\
\hline Total abstract item perseverations & $8.26(1.97)$ & $8.87(2.42)$ & 0.803 & 0.21 \\
\hline Digit Span & & & & \\
\hline Max. digits forward & $3.21(1.48)$ & $4.53(1.85)$ & 2.324 & $0.01 * *$ \\
\hline Max. digits backward & $2.05(1.22)$ & $2.33(1.80)$ & 0.541 & 0.29 \\
\hline Mean difference between max. forward and backwards & $1.16(1.16)$ & $2.20(1.52)$ & 2.262 & $0.04 \mathrm{a}$ \\
\hline & & & & \\
\hline & & & & \\
\hline$*=\mathrm{p}<0.05$ & & & & \\
\hline ** $=\mathrm{p}<0.01$ & & & & \\
\hline & & & & \\
\hline
\end{tabular}

Table 5 shows the mean differences for the deletion and UPD groups on EF tasks. The only significant difference between the two groups was on the letter F verbal fluency task $(\mathrm{p}<.05$, reported as two-tailed). Analyses of differences between the two sub-groups and the comparison group on performance on the TOL, the Selfordered pointing test and Digit Span shown in Table 6 indicates that the deletion group were more able at solving 5 move and more complex problems on the TOL than the comparison group and were more likely to make a higher number of abstract position perseverations in the Self-ordered pointing test.

A highly significant finding was that the deletion group were significantly poorer than the comparison group on Digit Span Forwards $(\mathrm{p}<.01)$. The UPD group were poorer than the comparison group but not significantly so.

It is important to point out that the large number of measures used in this study could lead to the possibility of a Type 1 error being made, that is a rejection of the null hypothesis when it is true. One approach to this problem is to reduce alpha level to 
0.01 , and in terms of alpha level the most robust findings are the differences found in Digit Span Forwards. As this is an exploratory study however it was felt important to report significant findings at a higher alpha level (0.05). In the ensuing discussion this issue will be referred to again, and appropriate caution will be used in discussing significant results reported at this higher alpha level.

It is also important to note that the very low number of participants in the UPD sample significantly reduces the power of these analyses and they have been reported because this is an exploratory study. 


\section{$\underline{\text { Table } 5}$}

Differences between means of EF tasks for deletion and UPD groups (independent $t$ tests, two- tailed)

\begin{tabular}{|c|c|c|c|c|}
\hline Measures & Deletion $(n=12)(\mathrm{sd})$ & $\mathrm{UPD}(\mathrm{n}=6)(\mathrm{sd})$ & $\mathrm{t}$ & sig \\
\hline \multicolumn{5}{|l|}{ Initiating behaviour } \\
\hline \multicolumn{5}{|l|}{ Verbal Fluency FAS } \\
\hline $\mathrm{F}$ & $2.82(2.82)$ & $6.00(3.23)$ & 2.116 & $0.05^{*}$ \\
\hline A & $2.36(2.25)$ & $4.00(3.58)$ & 1.167 & 0.26 \\
\hline $\mathrm{S}$ & $4.00(3.58)$ & $6.50(3.73)$ & 1.358 & 0.19 \\
\hline Total FAS & $9.18(8.12)$ & $16.50(9.85)$ & 1.650 & 0.12 \\
\hline Repeated words & $0.00(0.00)$ & $0.83(0.98)$ & 2.076 & 0.09 \\
\hline Errors & $0.09(0.30)$ & $0.17(0.41)$ & 0.438 & 0.67 \\
\hline \multicolumn{5}{|l|}{ Categories } \\
\hline Animals & $11.18(5.09)$ & $12.17(2.93)$ & 0.432 & 0.67 \\
\hline Objects & $9.91(5.84)$ & $13.67(6.22)$ & 1.241 & 0.23 \\
\hline Occupations & $6.73(3.04)$ & $10.33(5.54)$ & 1.756 & 0.09 \\
\hline Categories Total & $27.82(12.21)$ & $32.83(9.79)$ & 0.862 & 0.40 \\
\hline Category repeats & $0.55(0.93)$ & $1.00(1.27)$ & 0.332 & 0.41 \\
\hline Category errors & $0.00(0.00)$ & $0.00(0.00)$ & $\cdots$ & $\cdots$ \\
\hline \multicolumn{5}{|l|}{ Planning Ability } \\
\hline \multicolumn{5}{|l|}{ Tower of London TOL } \\
\hline \multicolumn{5}{|l|}{ TOL number of problems solved correctly in min. no of moves } \\
\hline 3 & $1.91(0.83)$ & $2.17(0.98)$ & 0.574 & 0.57 \\
\hline 4 & $1.55(1.04)$ & $2.17(0.75)$ & 1.287 & 0.22 \\
\hline 5 & $1.18(0.75)$ & $1.00(0.89)$ & 0.447 & 0.66 \\
\hline 6 & $0.27(0.47)$ & $0.17(0.41)$ & 0.466 & 0.65 \\
\hline 7 & $0.18(0.41)$ & $0.33(0.82)$ & 0.519 & 0.61 \\
\hline Total & $5.09(1.30)$ & $5.83(2.40)$ & 0.838 & 0.42 \\
\hline TOL most complex problems solved correctly in min no of moves & $5.45(0.93)$ & $5.00(1.09)$ & 0.769 & 0.38 \\
\hline \multicolumn{5}{|l|}{ Block Design } \\
\hline Raw Scores & $14.00(10.79)$ & $6.67(5.57)$ & 1.541 & 0.14 \\
\hline Age Scale Scores & $5.64(2.77)$ & $4.00(1.41)$ & 1.342 & 0.19 \\
\hline \multicolumn{5}{|l|}{ Set (Rule) Changing Ability and Inhibition } \\
\hline Luria Hand Game no of correct responses & $13.09(2.70)$ & $14.33(0.52)$ & 1.100 & 0.28 \\
\hline Rule Shift Card Test no of errors on rule 2 & $5.82(3.66)$ & $4.50(3.73)$ & 0.706 & 0.49 \\
\hline Spatial Reversal Task no of set (runs of 4 correct responses) & $3.18(1.08)$ & $2.50(1.23)$ & 1.189 & 0.25 \\
\hline \multicolumn{5}{|l|}{ Stroop - Day/Night Test } \\
\hline No. of errors in experimental condition & $1.64(3.35)$ & $2.67(5.57)$ & 0.480 & 0.63 \\
\hline No. of errors in control condition & $0.82(2.08)$ & $1.33(1.75)$ & 0.512 & 0.61 \\
\hline Time on experimental condition in secs. & $52.27(13.36)$ & $45.67(10.91)$ & 1.033 & 0.31 \\
\hline Time on control condition in secs. & $44.27(9.60)$ & $46.17(9.94)$ & 0.382 & 0.70 \\
\hline
\end{tabular}




\begin{tabular}{|l|l|l|l|l|}
\hline Working Memory & & & & \\
\hline Self-ordered pointing test & & & & \\
\hline Total concrete pages before error & $30.45(3.73)$ & $31.67(2.94)$ & 0.686 & 0.50 \\
\hline Total abstract pages before error & $19.00(4.19)$ & $17.83(1.17)$ & 0.658 & 0.52 \\
\hline Total concrete position perseverations & $5.00(2.72)$ & $6.83(5.04)$ & 0.987 & 0.33 \\
\hline Total abstract position perseverations & $4.82(2.96)$ & $5.00(3.29)$ & 0.117 & 0.90 \\
\hline Total concrete item perseverations & $2.64(1.63)$ & $2.67(1.03)$ & 0.041 & 0.96 \\
\hline Total abstract item perseverations & $8.09(2.02)$ & $9.00(1.67)$ & 0.910 & 0.36 \\
\hline Digit Span & & & & \\
\hline Max. Digit Span Forwards & $2.82(1.60)$ & $4.00(1.27)$ & 1.555 & 0.14 \\
\hline Max. Digit Span Backwards & $1.82(1.25)$ & $2.83(0.75)$ & 1.803 & 0.09 \\
\hline Mean difference between max Digit Span Forwards and Backwards & $1.00(1.18)$ & $1.17(1.17)$ & 0.279 & 0.78 \\
\hline & & & & \\
\hline$*=p<0.05$ & & & & \\
\hline & & & & \\
\hline
\end{tabular}

\section{Table 6}

Significance of mean differences for deletion and UPD groups compared with comparison group, on the measures that were significant or almost significant for the whole PWS group compared to comparison group (independent $t$ tests) $\mathrm{a}=$ two tailed i.e. better performance than comparison group contrary to experimental hypothesis $\mathrm{b}=$ one tailed i.e. poorer performance than comparison group consistent with experimental hypothesis $*=\mathrm{p}<0.05, * *=\mathrm{p}<0.01$

\begin{tabular}{|l|l|l|l|l|}
\hline Measure & \multicolumn{2}{l|}{ Deletion (n=12) } & \multicolumn{2}{l|}{ UPD (n=6) } \\
\hline & $\mathrm{t}$ & sig & $\mathrm{t}$ & $\mathrm{s}$ \\
\hline Tower of London & & & & \\
\hline $\begin{array}{l}5 \text { move problems solved } \\
\text { correctly }\end{array}$ & 2.373 & $0.02 \mathrm{a}^{*}$ & 1.350 & $0.19 \mathrm{a}$ \\
\hline $\begin{array}{l}\text { Total problems solved } \\
\text { correctly }\end{array}$ & 1.667 & $0.10 \mathrm{a}$ & 1.806 & $0.08 \mathrm{a}$ \\
\hline $\begin{array}{l}\text { Most complex problem solved } \\
\text { correctly }\end{array}$ & 1.949 & $0.06 \mathrm{a}$ & 0.929 & $0.36 \mathrm{a}$ \\
\hline & & & & \\
\hline Self-ordered pointing test & & & & $0.05 \mathrm{~b}^{*}$ \\
\hline $\begin{array}{l}\text { Total abstract position } \\
\text { perseverations }\end{array}$ & 1.859 & $0.03 \mathrm{~b}^{*}$ & 1.682 & \\
\hline & & & & $0.26 \mathrm{~b}$ \\
\hline Digit Span & 2.471 & $0.01 \mathrm{~b}^{* *}$ & 0.645 & \\
\hline Max. Digits Forward & & & \\
\hline
\end{tabular}




\section{Correlations of performance on EF tasks with behavioural measures and verbal}

\section{ability}

Table 7 shows the correlations for the whole PWS and comparison group, with the behavioural measures and verbal ability.

\section{$\underline{\text { Table } 7}$}

Correlations of performance on EF tasks with behaviour and Verbal IQ for the PWS and comparison groups. a) denotes significant results which are contrary to the hypothesis and are reported as two-tailed, otherwise all significant results are onetailed.

PWS $(n=19) \quad$ Comparison $(n=15)$

\begin{tabular}{|c|c|c|c|c|c|c|}
\hline Measures & $\mathrm{ABC}$ & DEX & VIQ & $\mathrm{ABC}$ & DEX & VIQ \\
\hline \multicolumn{7}{|l|}{ Initiating behaviour } \\
\hline \multicolumn{7}{|l|}{ Verbal Fluency FAS } \\
\hline $\mathrm{F}$ & 0.18 & 0.25 & 0.37 & -0.20 & -0.03 & $0.72 * *$ \\
\hline A & 0.18 & 0.26 & 0.38 & -0.19 & -0.04 & $0.79 * *$ \\
\hline S & 0.24 & 0.17 & 0.36 & -0.24 & -0.07 & $0.84 * *$ \\
\hline Total FAS & 0.22 & 0.24 & $0.39 *$ & -0.23 & -0.05 & $0.82 * *$ \\
\hline Repeated words & 0.33 & 0.31 & $0.39 *$ & -0.09 & 0.14 & $0.64 * *$ \\
\hline Errors & $0.68^{* *}$ & 0.47 & 0.02 & $0.46^{*}$ & 0.32 & $-0.50 *$ \\
\hline \multicolumn{7}{|l|}{ Categories } \\
\hline Animals & -0.05 & 0.16 & 0.29 & -0.05 & 0.17 & $0.62 * *$ \\
\hline Objects & -0.09 & 0.08 & $0.63 * *$ & -0.38 & -0.19 & $0.42 * *$ \\
\hline Occupations & 0.06 & 0.09 & 0.33 & -0.09 & 0.12 & $0.60 * *$ \\
\hline Categories Total & -0.17 & 0.07 & $0.40^{*}$ & -0.23 & 0.01 & $0.61^{* *}$ \\
\hline Category repeats & -0.01 & 0.08 & $0.50 * \mathrm{a}$ & 0.38 & -0.38 & -0.09 \\
\hline Category errors & $\cdots$ & $\cdots$ & $\cdots$ & 0.46 & 0.32 & $-0.50^{*}$ \\
\hline \multicolumn{7}{|l|}{ Planning Ability } \\
\hline \multicolumn{7}{|l|}{ Tower of London TOL } \\
\hline \multicolumn{7}{|l|}{$\begin{array}{l}\text { TOL number of problems solved correctly in min. } \\
\text { no of moves }\end{array}$} \\
\hline 3 & 0.32 & 0.26 & 0.17 & -0.07 & -0.02 & 0.26 \\
\hline 4 & 0.08 & 0.17 & $0.43^{*}$ & 0.25 & -0.15 & -0.16 \\
\hline 5 & -0.02 & -0.01 & -0.13 & -0.08 & -0.09 & 0.30 \\
\hline 6 & -0.33 & 0.01 & -0.17 & - & - & $0.65^{* *}$ \\
\hline 7 & -0.22 & 0.11 & -0.12 & -0.16 & 0.15 & $0.49^{*}$ \\
\hline Total & 0.07 & 0.26 & 0.19 & 0.03 & 0.11 & 0.34 \\
\hline $\begin{array}{l}\text { TOL most complex problems solved correctly in } \\
\text { min no of moves }\end{array}$ & -0.32 & 0.05 & -0.14 & -0.18 & -0.07 & 0.23 \\
\hline \multicolumn{7}{|l|}{ Block Design } \\
\hline Raw Scores & -0.17 & -0.17 & 0.34 & -0.33 & -0.04 & $0.54 *$ \\
\hline Age Scale Scores & -0.15 & -0.14 & $0.42^{*}$ & -0.33 & -0.03 & $0.48^{*}$ \\
\hline
\end{tabular}




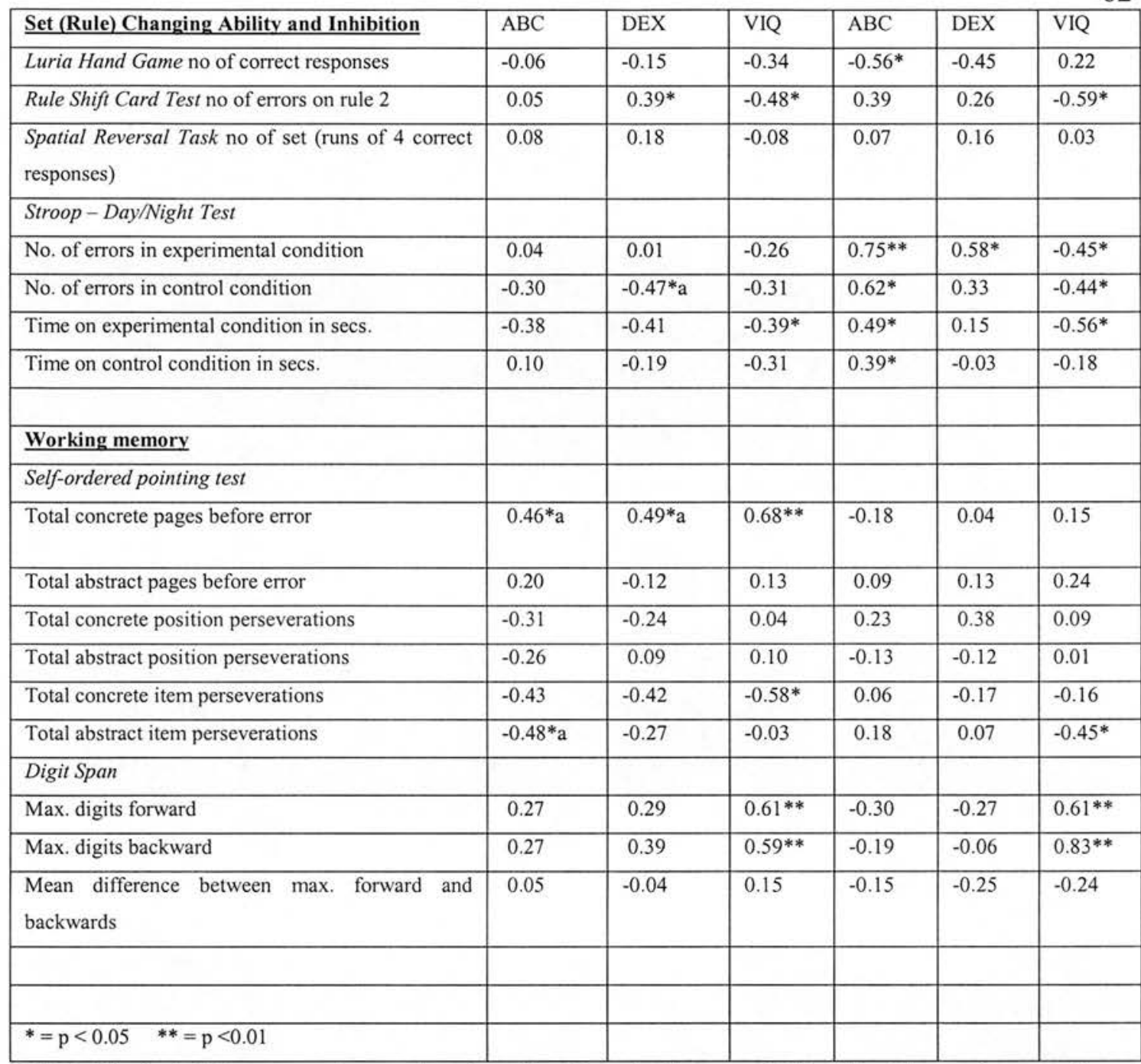

N.B. Verbal IQ was estimated using the Vocabulary and Similarities sub-tests from the WAIS-R.

A number of tasks correlated with verbal ability for both groups. The only tasks, which had no correlations with verbal ability for either group, were the Luria hand game and the Spatial reversal task. Performance on the TOL and Stroop type task were less affected by verbal ability for the PWS group.

The number of errors in the FAS verbal fluency task was positively correlated with the $\mathrm{ABC}$ for both groups (PWS at $\mathrm{p}<.01$, Comparison at $\mathrm{p}<.05$ ). The number of category errors was also positively correlated with the $\mathrm{ABC}$ for the comparison group $(\mathrm{p}<.05)$. In the Luria hand game the number of correct responses made by the 
comparison group were negatively correlated with the ABC $(\mathrm{p}<.01)$. The PWS group alone showed a positive $(p<.05)$ correlation of errors on rule 2 of the Rule Shift card and a negative correlation $(\mathrm{p}<.05)$, reported as two-tailed) of errors in the control condition of the Stroop task, with the DEX. In contrast the comparison group's performance on several aspects of the Stroop was positively correlated with the $\mathrm{ABC}$ and for the experimental condition errors only, the DEX.

In the Self-ordered pointing test the PWS group had positive correlations $(\mathrm{p}<.05$, reported as two- tailed) of pages before error on the concrete version with both the $\mathrm{ABC}$ and DEX.For the PWS group the number of abstract item perseverations were also negatively correlated with the $\mathrm{ABC}(\mathrm{p}<.05$,reported as two -tailed). 
Table 8 shows the correlations of the deletion and UPD groups with the behavioural measures and verbal ability

\section{$\underline{\text { Table } 8}$}

Correlations of performance on EF tasks with behaviour and Verbal IQ for the deletion and UPD groups (significant results consistent with the hypothesis are reported as one-tailed; a - denotes significant results contrary to hypothesis and reported as two-tailed; b - denotes significant result, $\mathrm{p}$ $=<0.05$, contrary to hypothesis but only with factor 1 of the $\mathrm{ABC}$ reported as two-tailed).

NB one participant was excluded from the analysis because of uncertainty about the presence of a deletion.

\begin{tabular}{|c|c|c|c|c|c|c|}
\hline & \multicolumn{3}{|c|}{ Deletion $(\mathrm{n}=12)$} & \multicolumn{2}{|c|}{ UPD $(n=6)$} & \\
\hline Measures & $\mathrm{ABC}$ & DEX & VIQ & $\mathrm{ABC}$ & DEX & VIQ \\
\hline \multicolumn{7}{|l|}{ Initiating behaviour } \\
\hline \multicolumn{7}{|l|}{ Verbal Fluency FAS } \\
\hline $\mathrm{F}$ & 0.05 & -0.05 & 0.46 & 0.22 & 0.61 & 0.02 \\
\hline A & -0.04 & -0.01 & 0.25 & 0.51 & 0.40 & 0.51 \\
\hline $\mathrm{S}$ & 0.26 & -0.01 & 0.43 & 0.15 & 0.44 & -0.01 \\
\hline Total FAS & 0.12 & -0.02 & 0.42 & 0.32 & 0.52 & 0.18 \\
\hline Repeated words & - & -- & -- & 0.71 & 0.44 & $0.71^{*}$ \\
\hline Errors & $0.80^{* *}$ & 0.36 & 0.01 & 0.56 & 0.67 & -0.08 \\
\hline \multicolumn{7}{|l|}{ Categories } \\
\hline Animals & -0.17 & -0.16 & 0.25 & 0.39 & 0.55 & 0.19 \\
\hline Objects & -0.38 & -0.29 & $0.76^{* *}$ & 0.40 & 0.50 & 0.23 \\
\hline Occupations & -0.15 & -0.29 & 0.11 & 0.33 & 0.32 & 0.49 \\
\hline Categories Total & -0.29 & -0.28 & 0.49 & 0.06 & 0.47 & -0.20 \\
\hline Category repeats & -0.14 & -0.09 & 0.48 & 0.16 & 0.06 & 0.49 \\
\hline Category errors & $\cdots$ & -- & - & $-\cdots$ & -- & $-\cdots$ \\
\hline \multicolumn{7}{|l|}{ Planning Ability } \\
\hline \multicolumn{7}{|l|}{ Tower of London TOL } \\
\hline \multicolumn{7}{|l|}{$\begin{array}{l}\text { TOL number of problems solved correctly in min. } \\
\text { no of moves }\end{array}$} \\
\hline 3 & 0.34 & 0.31 & 0.07 & 0.16 & -0.08 & 0.31 \\
\hline 4 & 0.05 & 0.50 & 0.46 & -0.13 & -0.67 & 0.56 \\
\hline 5 & 0.22 & -0.30 & -0.04 & -0.25 & 0.35 & -0.54 \\
\hline 6 & -0.36 & -0.08 & -0.05 & -0.23 & 0.10 & -0.59 \\
\hline 7 & -0.31 & -0.01 & 0.09 & -0.23 & 0.10 & -0.59 \\
\hline Total & 0.16 & -0.40 & 0.40 & -0.19 & -0.06 & -0.19 \\
\hline $\begin{array}{l}\text { TOL most complex problems solved correctly in } \\
\text { min no of moves }\end{array}$ & -0.40 & -0.18 & -0.02 & -0.06 & 0.38 & -0.52 \\
\hline \multicolumn{7}{|l|}{ Block Design } \\
\hline Raw Scores & -0.08 & -0.11 & $0.58^{*}$ & -0.19 & -0.23 & -0.23 \\
\hline Age Scale Scores & -0.04 & -0.01 & $0.65^{*}$ & -0.36 & -0.51 & -0.14 \\
\hline
\end{tabular}




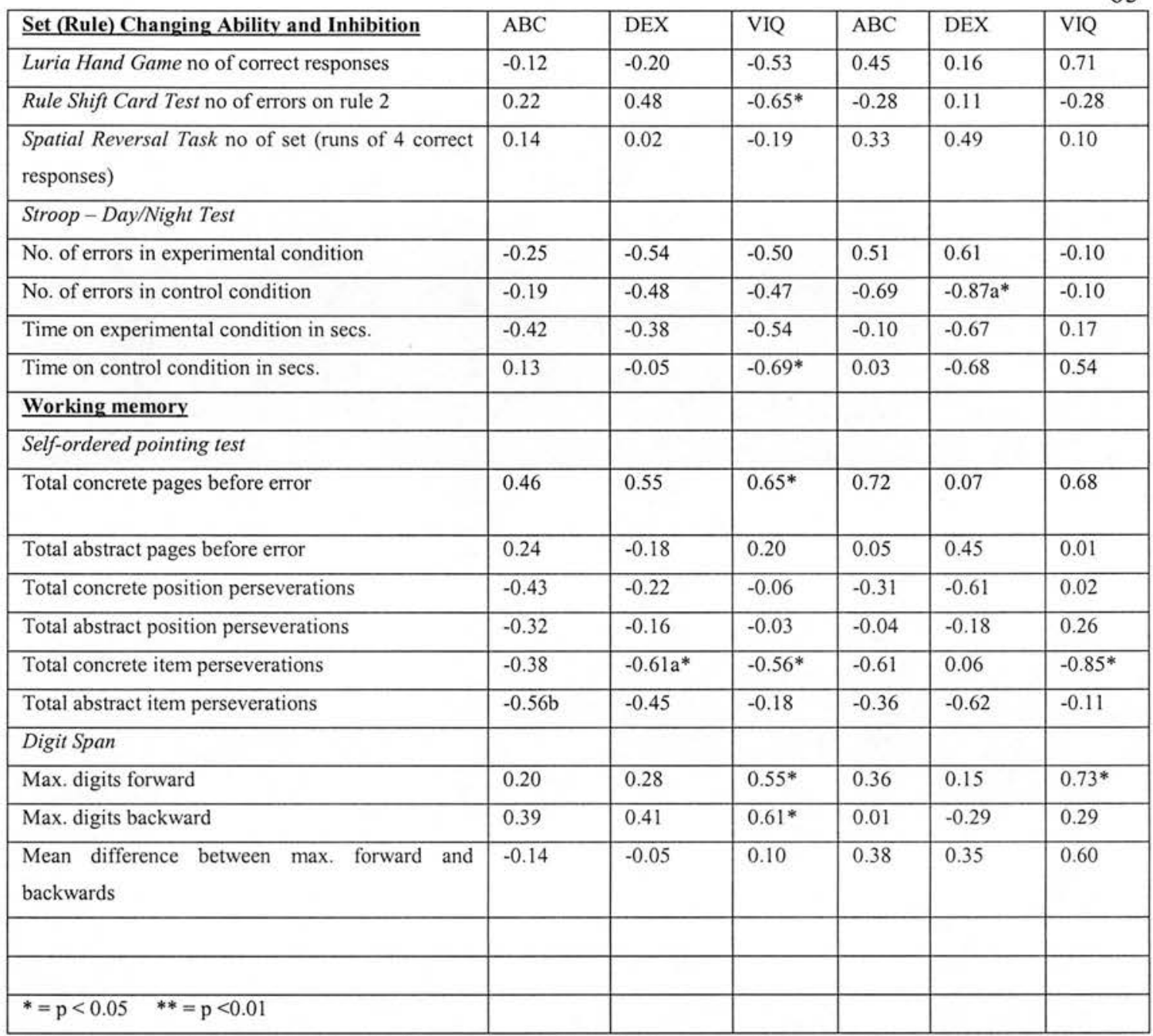

N.B. Verbal IQ was estimated using the Vocabulary and Similarities sub-tests from the WAIS-R.V

The correlations shown in this table indicate a small but potentially important number of differences between the deletion and UPD groups. In the deletion group there were more correlations of the EF tasks not only with the behavioural measures but also with verbal ability. The only EF task to correlate with a behavioural measure in the UPD group was the number of errors in the control condition of the Stroop task with the DEX, $(\mathrm{p}<.05)$, reported as two-tailed). FAS errors were positively correlated with the $\mathrm{ABC},(\mathrm{p}<.01)$ only in the deletion group. In the deletion group concrete item perseverations were negatively correlated with the DEX ( $p<$ .05 ,reported as two-tailed), whilst abstract item perseverations were negatively correlated with factor 1 of the $\mathrm{ABC}$ ( $\mathrm{p}<.05$,reported as two-tailed). 
As has been mentioned above, in the section describing differences in EF tasks, the large number of measures used in the study increases the risk of a Type 1 error being made. Reduction of the alpha level to 0.01 suggests that the most robust findings in terms of correlations are, for the PWS whole and deletion subtype groups, the positive correlation of FAS verbal fluency tasks errors with the $\mathrm{ABC}$, and for the comparison group the negative correlation of correct responses in the Luria hand game with the $\mathrm{ABC}$. Given the exploratory nature of the work however significant results at a higher alpha level have also been reported and will be treated with caution in the ensuing discussion.

It is also important to note that the low numbers of participants in both PWS subgroups but particularly in the UPD group significantly reduces the power of these analyses. Again they have been reported because of the exploratory nature of the study. 


\section{$\underline{\text { Discussion }}$}

In common with previous studies (Clarke et al 1996 and Holland et al 2003) the PWS group had a much higher mean score on the $\mathrm{ABC}$ than the comparison group. The mean total score on the $\mathrm{ABC}$ in the current study was higher than previous studies but this may reflect a bias in recruitment; the sample in the Holland et al study was widely drawn from a geographical region, whereas in the current study the participants were recruited from a PWS clinic. It is possible that even though the clinic was not specifically developed around the needs of those individuals showing difficult behaviour, families where this is a problem derive particular support from attending the clinic with their son or daughter.

There were also significant differences, with the PWS group having higher mean scores, on factors 3 - stereotypic behaviour, and 5 - inappropriate speech. Mean scores for factors 1 - irritability and agitation, and 2 - lethargy and withdrawal, almost reached significance. In the Clarke et al study, factors 1 and 5 were significantly different, but in the Holland et al study only factor 1 was significantly different. In the present study the UPD group had a higher mean ABC total score, which differs from findings that suggest the deletion group have more behavioural problems, however the UPD group in the current study was very small $(n=6)$ and as noted above may not be representative.

For both the PWS and comparison groups the DEX total scores were significantly correlated with the ABC total scores, $(\mathrm{p}<.01)$, but the PWS total mean score on the DEX, was not significantly higher than the comparison group; this suggests that the behavioural difficulties in PWS may not be attributable to difficulties in EF alone. As was the case for the ABC in this study, the UPD group were reported as having more EF problems as measured by the DEX than the deletion group. Relative to other clinical groups, both the PWS and comparison groups had high DEX scores. Evans et al (1997) obtained mean DEX scores for a group of schizophrenic patients, brain injured patients, and healthy controls which were $31.52,33.97$, and 11.25 
respectively. This supports the observation of Garner et al (1999) that EF deficits may be a feature of ID in general.

In contrast to the expectation that there would be a number of relative EF deficits in the PWS group, very few significant differences were found. The prediction of the hypothesis was that the PWS group would have relative difficulty on the EF tasks but it emerged that the PWS group had relative ability compared to the comparison group in the planning task, the Tower of London (TOL), at which the PWS group were more successful overall at solving problems correctly in the minimum number of moves. Numbers in the two PWS sub-groups were small, but the deletion group appeared able to solve difficult TOL problems more efficiently than the UPD group; a possible explanation for this will be discussed shortly. The TOL requires visuospatial abilities, but there were no significant differences between the groups on Block Design, which also requires visuospatial abilities. Unfortunately a problem with both the behavioural questionnaires used is that they measure problematic types of behaviour rather than adaptive types of behaviour such as planning ability. It is therefore not possible to state whether the relatively good performance of the PWS group on the planning task relates to real-life planning abilities. Interestingly however anecdotal evidence (Waters 1999) does suggest that at least in regard to obtaining food, individuals with PWS seem to have good planning skills. It is also important to note that some studies are uncertain as to whether the TOL and other tower tasks actually measure planning ability in the context of making plans, which then guide behaviour.

Both Phillips et al (1999) and Bull et al (2004) suggest that tower tasks can be solved by an on-line perceptual strategy rather than by look ahead planning. Bull et al suggest that young children do not have sufficient cognitive capacity and lack the metacognitive skills to use pre-planning in tower tasks. Interestingly however Phillips et al found no convincing evidence that pre-planning was being carried out by adults doing the Tower of London task even when they were encouraged to do so. Phillips et al suggest that most adult participants use a strategy of on-line planning in approaching tower tasks because it is as effective as a pre-planning strategy but less 
demanding. One of the reasons pre-planning time was not measured in the current study was because of this observation by Phillips et al and indeed, consistent with this observation, it was the author's recollection that participants in both the PWS and comparison groups began to move the blocks almost immediately after being shown the picture of the tower problem they were being asked to solve. This would suggest that both the PWS and comparison group participants used an on-line planning strategy to approach the TOL.

A highly significant finding was the poorer performance of the PWS group, particularly those with a deletion, on Digits Span Forward. The maximum length of Digit Span Backwards for both the PWS and comparison groups was low, but there were no differences between the groups on this task. The difficulty of the PWS group on the Digit Span Forwards task was in contrast to their relatively good performance on the Self-ordered pointing task, a visually presented test of working memory. The PWS group made significantly more position perseverations on the abstract version of the Self-ordered pointing task, but rather than being seen as a deficit, perseverating on the position of difficult to verbalise abstract diagrams, could be seen as an adaptive strategy; it had no affect on the number of abstract item perseverations made.

The relatively good performance on the Self-ordered pointing task, which is presented visually, compared to the difficulties on Digit Span Forwards, which is presented auditorally, underlines the importance of using visual strategies with the PWS population. Considering the satisfactory performance of the PWS group on the Self-ordered pointing task, it may be that the difficulty in Digit Span Forwards relates not to a relative deficit in the central executive process of working memory but rather to a problem in the capacity of the phonological store component of the phonological loop. If there had been relative problems with the central executive process of working memory then a poorer performance on the Self-ordered pointing task by the PWS group should have been found. As it was neither of the PWS subgroups appeared to have difficulty with the executive demands of this test. Conners et al (1998) have however reported that Digit Span Forwards is considered to be a 
measure of phonological loop capacity, which suggests that the PWS group, particularly the deletion sub-group may have a problem in this area. As Rosenquist et al (2003) have suggested that the lack of ability to develop rehearsal strategies is a general problem in ID it would seem that this is an unlikely explanation for the differences in performance on Digit Span Forwards and in the absence of a relative deficit in central executive processes in working memory, problems in the capacity of the phonological store may provide a better explanation.

The possibility that there could be a problem in the capacity of the phonological store may be particularly salient for the deletion sub-group who performed relatively poorly on Digit Span Forwards and invites further investigation. Evidence from positron emission tomography (PET) studies suggest that the phonological store is located in the left parietal lobe (Paulesu et al 1993); whereas maintenance of Digit Span Forward is carried out in the midventrolateral cortex (Owen et al 2000). In addition to the potential of scanning techniques, a number of further psychological methods could be used to investigate the capacity of the phonological store in PWS. In their work in Down syndrome Laws and Gunn (2004) have tested nonword repetition abilities, and Vicari et al (2004) have used a comparison of word spans for phonologically similar and dissimilar words. Both these methods are believed to provide information about the capacity of the phonological store, rather than other aspects of the phonological loop.

Phillips et al (1999) have observed that concurrent articulatory suppression enhances the performance of adults in the TOL; by interfering with verbal articulation the use of more efficient visuospatial strategies are promoted. One possibility that could explain the better performance of the PWS group, particularly the deletion subgroup, in the TOL is that articulatory suppression occurs quite naturally in visuospatial tasks because the phonological store is easily overloaded. This suggestion would be consistent with the superior jigsaw puzzle ability found in PWS and specifically in the deletion sub-group (Dykens 2002). It would also be consistent with the observation made by Whittington et al (2004a) that practice effects may be significant in respect of jigsaw puzzle ability in the deletion group. One way to 
investigate this hypothesis further would be to repeat Philips et al experiment with individuals with the deletion subtype of PWS and an appropriate comparison group. If articulatory suppression does occur naturally in the PWS deletion subtype it would be predicted that doing an articulatory suppression task at the same time as the TOL would make little difference to the performance of the PWS group on the TOL; one would however predict that the performance of the comparison group on the TOL would improve in line with the findings of Philips et al.

Before discussing the correlations of EF tasks with behaviour it may be helpful to comment on the approximate developmental level of the PWS and comparison group participants. The purpose of doing this is to see if there is variation in the equivalent developmental level at which the tasks are being performed. It is however not possible to assume that the tasks are being performed using the same EF abilities as the equivalent developmental age group; as earlier discussion has shown children may utilise different $\mathrm{EF}$ abilities and indeed different brain areas from adults doing the same or similar EF tasks. For example, as will be recalled from discussion in the literature review, Tamm et al (2002) have suggested that younger children use different brain areas from older children and adults to do the Go-No Go EF task, which is used as a measure of inhibition. Older subjects showed more focal activation in comparison with younger children who recruited more widespread regions of the dorsolateral cortex. Tamm et al felt that this was because younger children were also using working memory and set shifting abilities in addition to inhibition. Another example is provided by Bull et al (2004) who have suggested that visuospatial working memory may contribute more strongly to tower performance in adults than children where the role of inhibition is more prominent.

Reference to the Wechsler Intelligence Scale for Children- Revised Manual (Wechsler 1976), which is the child version of the WAIS-R from which Digit Span was taken, would suggest that the deletion group were performing more poorly on this task than the average for 6 year olds, whereas the UPD and comparison groups were performing at an approximately 6.5 year and 7year old level respectively. In contrast on the Self-ordered pointing task, the deletion, UPD and comparison group 
participants performed better overall on the concrete version of the task and were slightly better on some aspects of the abstract version (total number of abstract items, and position perseverations) than the 10 year old controls of average intelligence in the Temple et al (1996) study investigating EF in Turner syndrome.

The Self-ordered pointing task involves presentation of visual stimuli, which is not the case for Digit Span, and the apparent difference in equivalent developmental ability on these two tasks, which is particularly salient for the deletion group, lends support to the suggestion by Rosenquist et al (2003) that in ID the visuo-spatial sketchpad may be relatively intact compared to the phonological loop.

The apparently more efficient processing abilities of the deletion group on the TOL has already been discussed with reference to the possible role of (natural) articulatory suppression, but even the deletion group do not seem to attain the same equivalent developmental level on the TOL as achieved on the Self-ordered pointing task. Unfortunately differences in administration and scoring using various versions of the TOL hinder comparison, however a comparison of percentages of correctly achieved problems from the TOL data in a developmental study of EF (Levin et al 1991) indicates that the deletion group were performing as well as 7-8 year olds on the five move problems (classed as high complexity by Levin et al) but not as well as 9-12 year olds. The UPD group were not as able on five move problems as 7-8 year olds, and the comparison group were in turn poorer than the UPD group. In contrast on the four move problems (classed as medium complexity by Levin et al) the deletion group were performing as well as 9-12 year olds, and the UPD group as well as 1315 year olds. The comparison group were as able as 7-8 year olds but not as able as 9-12 year olds on four move problems. This comparison is also consistent with the TOL data in Temple et al (1996) in that the most efficient of the two PWS sub-types, the deletion group, were not as able as the 10-year-old controls used in that study on five move problems. This comparison suggests that the PWS groups could manage the four move problems with relative ease but found the five move problems more of a challenge, and have not attained the incremental increase in EF ability noted by Anderson et al to occur between the ages of 11-12 years. Data on verbal fluency - 
animal naming - reported by Levin et al (1991) indicates that neither the PWS subtype groups nor the comparison group were able to name as many animals as typically developing 7-8 year olds. This is also consistent with Temple et al (1996). A comparison of performance in the Day- Night Stroop type task with the data reported by its authors Gerstadt et al (1994) would suggest that the deletion group were almost performing as well as typically developing 7 year olds but the UPD and comparison groups were performing at a level better than 5.5 year olds but not as well as 6 year olds.

Overall this brief comparison with the developmental literature would indicate that apart from the deletion group showing a 'delay' in Digit Span, the equivalent developmental levels of the PWS and comparison groups were broadly similar; in a number EF of tasks the PWS group were developmentally more advanced than the comparison group and there was little evidence to support the suggestion of Holland et al (2003) that arrested development in PWS may be associated with the behaviour problems characteristic of the syndrome.

The correlations of EF tasks with behaviour and verbal ability lend some indirect support to the finding that in PWS, particularly for those with deletions, the visuospatial components of working memory may be working more efficiently than the phonological loop. Overall there were notably weaker correlations of EF ability with verbal ability in the PWS group than the comparison group, which suggests that the participants with PWS were processing information in a different way. In the deletion group the positive correlation of verbal fluency errors with the $\mathrm{ABC}$ could be associated with working memory difficulties for verbal material related to the functioning of the phonological loop. Errors occur when words beginning with different letters from the target letters F, A, or S, were given by participants, suggesting that the target letter was not being refreshed by rehearsal processes or stored in the phonological loop. Poorer performance on a number of EF tasks was negatively correlated with verbal ability for the deletion but not the UPD group. This suggests that for the deletion group the development of some EF abilities may be linked to the development of language as suggested by Hughes and Graham (2002) 
The study produced a surprising finding in that for the deletion group there were negative correlations of both abstract and concrete item perseverations in the Selfordered pointing task with the aggression and irritability factor of the $\mathrm{ABC}$, and DEX, respectively. Correlation does not imply causation but this finding supports the evidence described above which suggests that those with deletions primarily use the visuospatial sketchpad rather than verbal information in the phonological loop to guide behaviour. Holland et al (2003) describe how outbursts commonly occur in PWS when family members or carers change an individuals' plans or expectations. This usually takes place in the context of a family member/carer telling the individual with PWS about a change to plans/expectations. Due to a relative impairment in the phonological loop, as suggested in the earlier discussion, difficulties in dual tasking if (auditorally presented) verbal material is introduced may occur, leading to the disruption of visually mediated plans and possibly resulting in a behavioural outburst.

Whilst reliance on visuospatial working memory may contribute to the behavioural problems found in PWS it does not adequately account for their disproportionate level, as problems in the phonological loop are also reported in Down syndrome which is not characterised by the same type or level of behavioural difficulties (Jarrold et al 2000).

There is evidence that EF tasks, have a greater association with dorsolateral as opposed to other parts of the pre-frontal cortex. Scanning studies have implicated the involvement of the dorsolateral cortex in the Tower of London Task (Baker et al 1996, Cazalis et al 2003). Self-ordered pointing and other working memory tasks are also believed to engage the dorsolateral cortex (Petrides 1998).

Whilst no measures of motivation or test anxiety were investigated during the study, it is relevant to mention at this point, that during the study planning stage the author was warned by a parent and senior member of the PWS UK society, that it would be extremely difficult to do the proposed testing because of the likelihood of temper tantrums from the PWS participants. In fact there were no such problems, and whilst 
a few PWS participants demonstrated irritability towards their parents prior to testing, once they started testing their motivation to complete it was extremely good. Participants had no problems with moving from one type of test to another and indeed after testing many participants said they had enjoyed the challenge. The author had expected that the PWS participants might find the more difficult TOL problems somewhat frustrating but in fact the subjective impression of the author was that this test was one the PWS participants in particular, especially enjoyed doing. This subjective impression is consistent with the observations of Dykens (2002) who reports that individuals with PWS like doing various types of puzzles, especially jigsaw puzzles. Whilst EF tasks present with a cognitive challenge, they place relatively minor demands on systems of affect, at least compared to the emotional hurly-burly of everyday (family) life where temper tantrums from individuals with PWS are frequently reported.

The current study indicates that although individuals with PWS do not have relative difficulty with tasks associated with the dorsolateral cortex, it is possible that in PWS another region of the pre-frontal cortex that is more involved in affective functioning may be involved. One candidate region could be the orbitofrontal cortex (OFC) and closely linked ventromedial cortex. The OFC projects to the limbic system including the hypothalamus, and is involved in the modulation of emotion (Rolls 1999). Problems in the OFC can give rise to abnormalities in the ability to engage the appropriate emotions in relation to social situations and events and may occur even when adequate social knowledge is available (Bechara et al 2000, Elliot et al 2000). Zelazo and Muller (2002) have recently described the EF functions associated with the dorsolateral cortex as "cool" aspects in contrast to the "hot" aspects associated anatomically with the OFC and pertaining to affective decision-making, or decisionmaking about events, which have emotionally significant consequences. One commonly used measure that is believed to require affective decision-making is the Iowa Gambling Task (Bechara et al 1994), which is now also available in a version suitable for children, the Children's Gambling Task (Kerr and Zelazo (2004). In their initial study Bechara et al (1994) OFC patients and controls were presented with four decks of cards which when turned revealed a combination of gains and losses (in toy 
money!). The participants were given an initial stake and were then told to win as much money as they could by selecting cards from any of the four decks at the rate of one card per trial. The participants did not know the number of trials (100) but were told that some of the decks were better than others. Selecting cards consistently from two of the decks actually resulted in a net gain (advantageous decks), whereas selecting cards from the remaining two resulted in a net loss (disadvantageous decks). Cards from the disadvantageous decks provided higher rewards than cards from the advantageous decks, but the losses associated with cards from the disadvantageous decks were also greater than losses from cards in the advantageous decks. The study showed that initially both the OFC patients and controls chose cards from the disadvantageous decks (associated with higher rewards) but over trials the controls selected cards from the advantageous decks and hence made more money in the long term, whereas the OFC patients continued to select cards from the disadvantageous decks and made greater long term losses. Bechara et al (2000) suggest that the failure of the OFC group on this task can be explained by the somatic marker hypothesis (Damasio, 1994, 1996). The essence of the somatic marker hypothesis is that somatic markers i.e. representations of body states, link previous behaviours and situations with contingent affective consequences. In the gambling task patients with OFC damage fail to anticipate future outcomes. Bechara et al (2000) suggest that this is because they have impaired ability to re-experience an emotion from the recall of an appropriate emotional event. They suggest this weakness contributes to the failure to trigger somatic states when considering options for a decision. Bechara et al (2000) investigated whether this weakness was associated with a defect in acquiring fear conditioning but found that their patients could be conditioned and suggested that their decision-making impairments could not be explained by a failure to acquire conditioned emotional responses.

In a recent review on the functional neuroanatomy of the OFC, Kringelbach and Rolls (2004) challenge the somatic marker hypothesis and suggest that the OFC is involved in emotion because 
"it represents the reward and punishment value of primary (unlearned) reinforcing stimuli , and because it is involved in the rapid relearning and reversal of associations between previously neutral stimuli and primary reinforcers."(p21)

Kringelbach and Rolls further suggest that the medial OFC is important in the decoding and monitoring of the reward value of reinforcers, whereas the lateral OFC areas are important in evaluating punishers which when detected may lead to a change in current behaviour; abstract reinforcers are represented in the more anterior parts of the OFC in contrast to simple reinforcers which are represented in the posterior areas.

Arrested development of the orbitofrontal cortex in PWS may interfere with the ability to provide the appropriate emotional responses to unexpected situations, e.g. a thwarted plan or routine, even though relatively good cognitive skills are present. Further indirect evidence that the orbitofrontal cortex may be implicated in the behaviours seen in PWS is provided by studies which show that this region of the brain is involved in the types of behaviour which may be problematic in the syndrome, such as compulsive behaviours (Volkow and Fowler, 2000, Choi et al, 2004), mood and affective disorders (Drevets, 2001), and in applying verbal learning strategies in novel situations (Savage et al 2001). Holsen and Thompson (2004) have recently shown that in PWS there is a higher eye-blink rate, which in turn is linked to compulsive behaviour. They point out the link between compulsive behaviour and the OFC and also discuss evidence linking eye-blink rate to OFC function. As discussed above the EF tests used in the current study are more sensitive to dorsolateral functioning rather than that of the OFC. Links between impaired OFC functioning and behaviour in PWS could potentially be investigated by use of a "gambling task".

\section{Limitations of the study}

There are a number of limitations to the present study. The two main limitations are the number of measures used and the sample size. As described in the Results section the large number of measures used increased the chances of Type 1 error being 
made, that is the null hypothesis is rejected when in fact it is true. By reducing the alpha level to 0.01 the most robust findings for the PWS group were the differences in Digit Span forwards and in the correlation between FAS errors in the verbal fluency task and the behavioural measures. The results reported at the 0.05 alpha levels, particularly those concerning better TOL ability in the PWS group, and correlations between the Self-ordered pointing task and the behavioural measures should cautiously be regarded as trends towards significance. As this is an exploratory study however it was felt important to report these less robust results especially as in the case of the TOL, and the Self-ordered pointing task they were not in the direction of the hypothesis predictions. The TOL was used in the current study as an EF planning task but as has been mentioned previously both Philips et al (1999) and Bull et al (2004) have emphasised the visuospatial nature of the task; given the relative ability of individuals with PWS deletion subtype on other visuospatial tasks e.g. jigsaw puzzles (Dykens 2002) it would seem appropriate to investigate in greater depth TOL ability in PWS. Further work in this area would however hypothesise relative ability in a PWS group rather than deficits as predicted by the current study.

The second main limitation of the study concerns the sample size of the participant groups. As described in the Methods section the statistical power of the study was low. The number of participants in the UPD group was particularly low reflecting the lower prevalence of this sub-type, however given the differences between the deletion and UPD groups reported in the literature it was felt important to analyse this data in the current study. Effect sizes were estimated for a number of the tests used and by reference to the tables in Clark-Carter (1997) it was found that to achieve power of 0.8 it would be necessary to have around 30 participants in each of the deletion and UPD groups. The problem that having a low sample size brings is that there is an increased risk of a Type 2 error being made; that is the null hypothesis is accepted when in fact it is false. It is possible that with a larger sample relative deficits would have been found in EF performance in the PWS group. It would be important for further work in this area to include a larger sample size; one method of achieving this would be through collaboration with research groups from different geographical areas. A slightly mitigating factor in the present study, which 
could make the possibility of a Type 2 error less likely, is that the PWS group had relatively high rates of behaviour problems compared to other studies; that is if $\mathrm{EF}$ deficits are a feature of PWS one might expect to have found them in this group. This assumption however cannot be used as an excuse for not in the future attempting to involve a larger sample of participants.

In addition to these limitations the study also suffers from the general methodological problems of EF assessment that have been discussed earlier, particularly in respect of validity. Whilst every attempt has been made to use tasks of known validity from the developmental and adult EF literature, the paucity of a similar literature in ID does require the assumption that the tasks used are also valid as EF tasks in an ID population. It would be helpful if further work on a range of ID populations could be carried out to determine the validity of different types of EF task in ID.

It is also important to consider whether the EF measures used were useful in investigating the particular types of behaviour seen in PWS. As noted in the literature review individuals with PWS often become very irritated if things to do not happen when they expect them to. It is possible that their difficulties in inhibiting immediate responses in order to obtain later goals may not have been tapped by the measures used. It might have been useful to include Maze type tasks e.g. the Porteus Maze test (Porteus1965). These types of task are involved in planning and particularly the ability to select alternative plans. This issue has also been discussed, and is further mentioned below in the section on future research, in the context of whether EF tasks are associated with those parts of the brain believed to be involved in affective processing.

A further limitation of the study was that the age of the PWS participants was skewed towards a mean age of 23.5 years. Only one participant was over the age of 40.This uneven distribution of age is by no means unusual in adult PWS research and probably relates to the high mortality rate found in the syndrome (Dykens 2004). As described earlier, Dykens (2004) reports that rates of behaviour problems decrease 
with age so one cannot generalise the results from the present study to the entire adult PWS population. Dykens was fortunate in that she had access to a large sample of individuals with PWS in the U.S.A. and one approach to this problem is again to consider collaboration between research groups from different areas. This would enable cross-sectional investigation of behaviour and cognitive ability in different age bands. Another approach would be to carry out a longitudinal study, but a major limitation to this approach would of course be the amount of time it would take to complete.

A further limitation of the study concerns the composition of the comparison group. One of the participants in comparison group had a diagnosis of Autistic Spectrum Disorder, which as Pennington and Ozonoff (1996) have noted is characterised by fairly specific EF deficits. In future research it would be important to exclude such individuals in case they obscure differences in EF performance between PWS and ID participants who may have more global impairments. Alternative (or additional) comparison groups could, in future research, comprise individuals from other syndromes associated with ID, which share a common cognitive or behavioural characteristic with PWS. One such possibility would be to use a comparison group comprising individuals with Down syndrome who also tend to perform poorly on Digit Span Forwards (Jarrold et al 2000).

In addition to the limitations described above, the study has a number of other weaknesses. One of the variables that may be associated with behaviour problems in PWS is sleeping problems, which may be related to the functioning of the hypothalamus (Richdale 1999). In the current study no data regarding sleeping problems were collected. None of the participants discontinued testing because of tiredness, indeed as mentioned above the motivation of the PWS group was surprisingly good, however in future work this variable could be included. Even though the predicted EF deficits were not found in the present study it is possible that sleeping problems may have affected the scores of the behavioural measures. Other variables that were not taken into account were medication and hormone treatments; they were not included in order to reduce the large number of measures already 
collected in this small sample. Various kinds of hormone treatment are given in PWS in order to encourage linear growth, increases in muscle mass and the development of secondary sexual characteristics (Donaldson 2002), they are not given to moderate behaviour. In respect of future work to investigate functioning of the OFC in PWS, it would however be important to include the possible influences of hormone levels and gender on behaviour and cognitive ability in PWS, at different stages of the lifespan; it has recently been reported that male children under the age of three do significantly better than females on a task sensitive to the functioning of the OFC (Overman 2004). Overman suggests this may be due to hormonal influences on brain development.

\section{Further Research}

In this short section a number of ideas for future research will be considered some of which have already been discussed. In order to avoid repetition is assumed that in future research the limitations discussed above will be overcome.

The most robust statistical finding of the study was that the deletion sub-group had particular difficulty with Digit Span Forwards suggesting problems in the capacity of the phonological store (Connors et al 1998). That they did not have problems with the executive demands of the visually mediated Self-ordered pointing test, further supports the suggestion that there may be a problem in auditory processing in the deletion sub-type of PWS.It would therefore seem reasonable that one important aim of future research should be to establish if there is a problem in the capacity of the phonological store in PWS, particularly in the deletion sub-type. As has been discussed this could be carried out using similar types of approaches to those used in Down syndrome to investigate the capacity of the phonological store (Laws and Gunn 2004, Vicari at al 2004).

A less robust but interesting finding, in that it was contrary to the hypothesis, was that the PWS group, particularly the deletion sub-group appeared to be relatively more efficient at solving problems on the Tower of London (TOL). The TOL is a visuospatial planning task and that the PWS group did relatively well on this task 
was consistent with the work of Dykens (2002) who found that individuals with PWS, particularly the deletion sub-type are good at visuopatial tasks like jigsaw puzzles. In the current study it was hypothesised that the PWS group would have relative difficulty on the TOL because it is an EF task and a straightforward aim of future research could be to investigate the hypothesis that the PWS group are indeed more efficient at solving the TOL. It would also be interesting however to include concurrent articulatory suppression tasks, which have been found to enhance TOL performance in adults (Philips et al 1999). The purpose of this would be to investigate the hypothesis that individuals with PWS, particularly those with the deletion sub-type, already 'use' articulatory suppression quite naturally to do visuospatial tasks because of problems in the phonological loop. The hypothesis would be that being given a concurrent articulatory suppression task would not enhance the performance of individuals with PWS, particularly those with a deletion, on the TOL.It would also be helpful to research whether ability on the TOL may relate to planning abilities in everyday life. As noted earlier a problem with behavioural assessments is that they are designed to pick up deficits rather than abilities. It would therefore be necessary to design a behavioural instrument that focussed on everyday planning ability and compare performance on that with performance on the TOL.One area of everyday planning that would be of interest is the ability to obtain food. Parents, carers, and professionals working with individuals are often amazed at the planning skills they can display in the acquisition of food.

The suggestions for future research discussed so far are mainly concerned with further investigating cognitive processing in PWS with a particular focus on elucidating the relative role of the two slave systems in working memory. As has been discussed however, the behaviour problems experienced by parents/carers may be related to problems in the modulation of emotion by individuals with PWS. It has been suggested that the orbitofrontal cortex (OFC), which is known to be important in the modulation of emotion, may be implicated. One type of task that may be useful in ' testing' the OFC are the gambling tasks, which are believed to require affective decision-making abilities. Further research could investigate the performance of individuals with PWS on one of these gambling tasks, which are available in adult 
(Bechara et al 1994) and child (Kerr and Zelazo 2004) versions. The hypothesis would be that a PWS group would perform similarly to OFC patients on this type of task and make greater long-term losses by choosing cards from disadvantageous decks. As the author has hypothesised that there may be a delay in the maturation of the OFC in PWS and given Dykens (2004) observations that behaviour problems appear to decrease with age, it would be sensible to do this particular aspect of future research on different age bands. The hypothesis being that older individuals with PWS i.e. those over the age of thirty, would do better on a gambling task than adolescents and young adults because of greater maturation of the $\mathrm{OFC}$ in older individuals.

\section{Clinical Implications}

Whilst further research is required, the present study does have some clinical implications for individuals with PWS, their families and carers.

In common with other studies, the present study found that PWS is a syndrome associated with an elevated level of behaviour problems (Clarke et al 1996,Einfeld 1999,Holland et al 2003). This is clinically important for at least two reasons. Firstly by making this knowledge known to families and carers it may help them feel less to blame for their son or daughters behaviour, and by doing so perhaps reduce family stress. Whilst the author did not measure family stress in the present study, many of the PWS families did volunteer that life could often be extremely difficult due to their son/daughters behaviour and in a number of families this had led to marital breakdown. Secondly the finding of elevated behaviour problems does have implications for support and service provision for people with PWS.It suggests that PWS families/carers should at least be offered input and support from clinical psychology with a view to alleviating behavioural problems. Dykens and Cassidy (1996) have suggested that such support is best given in the context of multidisciplinary working given the complex range of problems an individual with PWS might have. In the UK, community learning disability teams provide such a model of multidisplinary working. These teams usually include clinical psychology, nursing and speech and language therapy as well as other professions. A problem in 
accessing community teams however, is that they can be quite rigid about excluding people whose IQ is over 70 irrespective of individual support needs. As a number of individuals with PWS have an IQ over 70 it may be the case that in some areas they would be unable to access the services provided by community learning disability teams. It is the author's opinion that community teams could be flexible in regard of access to their services so that individuals with PWS and their families can receive the support they need. Most community teams work reactively in that they deal with problems once they have occurred. In the case of PWS (and indeed other syndromes with behavioural/health risk factors) it might be helpful if work with families could be carried out proactively to prevent behaviour problems reaching crisis point. To achieve that it would probably be necessary to enhance the resources of community teams and also ensure that there was in place a register or database enabling community teams to become aware of individuals with PWS living in team areas.

A further clinical implication of the present study is that it confirms earlier findings, which suggest individuals with PWS have difficulty processing auditory information (Curfs et al 1991). The implication of this finding is that despite the relatively good language abilities of individuals with PWS it may be helpful to use visual communication strategies with them in order to support spoken communication. The type of visual communication strategy used would require to meet individual needs and should ideally be based on assessment by a speech and language therapist.

An interesting finding of the study, which also has clinical implications, was the observation, consistent with other work, suggesting that the deletion sub-type have relative strengths in visuospatial processing (Dykens 2002). This finding coupled with the implication regarding auditory processing is clinically important because it suggests that at least for the deletion group their learning and skill development may be enhanced by being in a learning or work environment where there is an opportunity to use their visuospatial skills. By experiencing an environment where they can feel successful one would hope to alleviate feelings of low self-esteem and mood for these individuals. 
An important although unexpected finding of the study was that despite having more behavioural problems than the comparison group the PWS group did not have relative deficits in EF.This suggests that the behaviour observed in PWS which resembles that seen in groups with clearly documented EF deficits may be associated with other factors. It would suggest, for example, that the preference for routine may not be associated with a difficulty in disengaging from the immediate environment as has been hypothesised to be the case for autistic spectrum disorder (Pennington and Ozonoff 1996) but may be due to a difficulty in the modulation of emotion when anticipated events are changed. Further research needs to be carried out to investigate whether the OFC is functioning abnormally in PWS in respect of emotion modulation but in the meantime it would seem reasonable to suggest to families/carers that when communicating a change of an anticipated event to an individual with PWS they do this in a calm but firm way and avoid reacting emotionally themselves to any consequent display of temper from the individual concerned. The aim of this approach being to avoid escalation of the situation. The author, in his personal experience of working with affected families has found this advice to be helpful.

\section{Conclusions}

The study has revealed a number of interesting findings that were not predicted by the hypothesis as well as a few which were. In comparison to a group of adults with similar language abilities the PWS participants had higher rates, consistent with the literature, of behaviour problems as measured by the $\mathrm{ABC}$; they did not however have higher rates of Executive Dysfunction behaviour as reflected by scores on the DEX. Contrary to the study hypothesis, the PWS participants did not have relative difficulty with EF tasks and indeed in one task, the TOL, showed a trend towards significance indicating relative ability in this type of task. The most robust statistical finding in terms of differences in test performance was the relatively poorer performance of the PWS group, particularly the deletion sub-type, on Digit Span Forwards. Digit Span Forwards is not regarded as an EF task and as discussed earlier may point to problems in the phonological loop. The correlation of errors in the FAS 
verbal fluency task with $\mathrm{ABC}$ scores for the deletion sub-type is also supportive of the suggestion that there may be problems with the phonological loop in PWS.

It is acknowledged that the study has some serious limitations in terms of having used a large number of measures on a small sample of participants, risking the possibility of Type 1 and Type 2 errors. It would be important that steps are taken to rectify these limitations in future work. Future work could also include other relevant variables such as sleep, and hormone levels.

Bearing in mind the major limitations of the study, it is important to be cautious about overstating the importance of the interesting and unexpected correlations that were found between performance on the Self-ordered pointing task and the ABC and the DEX in the deletion subtype. That better performance on this task was related to a reported increase in behaviour problems suggests the intriguing possibility that, in at least the deletion sub-type of PWS, there is reliance on the visuospatial sketchpad slave system of working memory, which when that is interrupted by a (auditorally presented) change to what is expected/planned gives rise to problems of emotion modulation resulting in a temper outburst. It is suggested that problems may occur either because the phonological loop cannot cope with more information than the limited store can hold and/or difficulties in the function of the OFC, which modulates emotion. This hypothesis is supported by behavioural observation (Holland et al 2003) but requires further work to substantiate from a cognitive processing perspective. An initial step as outlined above would be to investigate functioning of the phonological loop in PWS.

Another speculative suggestion of this study is that a relatively impaired phonological loop but a relatively intact visuospatial sketchpad in the deletion subtype may be responsible for their apparent ability in visuospatial tasks such as jigsaw puzzles.

The present study has failed to find relative EF deficits in PWS but has suggested that a non -executive cognitive process - the phonological loop - and possibly a 
region of the brain involved in affective functioning - the OFC - may be implicated in the behaviour problems found in PWS. It is anticipated that further work may be able to clarify these issues and provide greater understanding of the spectrum of behaviour found in this fascinating syndrome. 


\section{$\underline{\text { References }}$}

Akefeldt A., Gillberg C. (1999) Behaviour and Personality Characteristics of Children and Young Adults with Prader-Willi Syndrome: A Controlled Study Journal of the American Academy of Child and Adolescent Psychiatry 38, 761-769.

Aman M., Field C., Singh N., Stewart A. (1985a) The Aberrant Behavior Checklist: A behaviour rating scale for the assessment of treatment effects. American Journal of Mental Deficiency 89,485-491.

Aman M., Field C., Singh N., Stewart A. (1985b) Psychometric Characteristics of the Aberrant Behavior Checklist. American Journal of Mental Deficiency. 89,492-502.

Aman M., Singh N. (1994) The Aberrant Behavior Checklist-Community. Slosson Educational, East Aurora, N.Y.

Anderson P., Anderson V., Lajoie G. (1996) The Tower of London Test: Validation and standardisation for pediatric populations.

The Clinical Neuropsychologist 10, 54-65.

Baddeley A.D. (1986) Working memory. Clarendon Press, Oxford.

Baddeley A.D., Hitch G. (1974) Working memory. In Bower I. (ed) The Psychology of learning and motivation. 47-90. Academic Press. New York.

Baker.S, Rogers.R, Owen. A, Frith.C, Dolan. F, Frackowiak, R.S, Robbins, T. (1996) Neural systems engaged by planning: A PET study of the Tower of London Task Neuropsychologia 34, 515-526

Ball R., Espy K.A., Senn T.E., (2004) A comparison of performance on the Towers of London and Hanoi in young children. Journal of Child Psychology and Psychiatry. 45, 743-755.

Barkley R.A. (1996) Linkages between attention and executive functions. In Lyon G.R., Krasnegor N.A. (eds). Attention, memory and executive function. 307-326. Brookes, Baltimore.

Beardsmore A., Dorman T., Cooper S-A., Webb T. (1998) Affective Psychosis and Prader-Willi syndrome. Journal of Intellectual Disability Research 6, 463-471.

Bechara. A, Damasio. H, Damasio.A., Anderson S. (1994) Insensitivity to future consequences following damage to human prefrontal cortex.

Cognition 50, 7-15. 
Bechara. A, Damasio. H, Damasio.A, (2000)

Emotion, decision-making and the orbitofrontal cortex.

Cerebral Cortex 10, 295-307.

Bishop D., Aamodt-Leaper G., Creswell C., McGurk R., Skuse D. (2001) Individual differences in cognitive planning on the Tower of Hanoi Task: Neuropsychological maturity or measurement error?

Journal of Child Psychology and Psychiatry 42, 551-556.

Boer H., Holland A.J., Whittington J.E., Butler J.V., Webb T., Clarke D.J. (2002)

Psychotic illness in people with Prader-Willi syndrome due to chromosome 15 maternal uniparental disomy.

Lancet 359, 135-136.

Burgess P. (1997) Theory and methodology in executive function research. In Rabbitt P. (ed) Methodology of frontal and executive function. 81-116. Psychology Press, Hove.

Cassidy S.B., Schwartz S. (1998)

Reviews in Molecular Medicine: Prader- Willi and Angelman syndromes Disorders of Genomic Imprinting

Medicine 77, 140-151.

Cazalis F., Valabregue R., Pelegrinin- Issac M., Asloun S., Robbins T.W., Granon S. (2003) Individual differences in prefrontal cortical activation on the Tower of London planning task: implication for effortful processing.

European Journal of Neuroscience 17, 2219-2225.

Choi.J-S, Kang. D-H, Kim. J-J, Ha. T-H, Lee.J-M, Youn.T, Kim. I.Y, Kim.S.I, Kwon.J.S (2004) Left anterior subregion of orbitofrontal cortex volume reduction and impaired organisational strategies in obsessive-compulsive disorder.

Journal of Psychiatric Research 38, 193-199

Clarke D.J., Boer H. (1998) Problem behaviours associated with deletion PraderWilli, Smith- Magenis and Cri Du Chat syndromes

American Journal on Mental Retardation 40, 264-271.

Clarke D.J., Boer H., Chung M.C., Sturmey P. and Webb T. (1996) Maladaptive Behaviour in Prader-Willi syndrome in adult life. Journal of Intellectual Disability Research 40, 159-165.

Clarke D.J.,Boer H., Webb T., Scott P., Frazer S., Vogels A., Borghgraef M.,Curfs L. (1998) Prader- Willi syndrome and psychotic symptoms:1. Case descriptions and genetic studies. Journal of Intellectual Disability Research 42, 440-450. 
Clarke D.J.,Boer H., Whittington J.E., Holland A.J., Butler J., Webb T. (2002) Prader-Willi syndrome, compulsive and ritualistic behaviours: the first populationbased survey.

British Journal of Psychiatry 180, 358-362.

Clark-Carter D. (1997) Doing quantitative psychological research. Psychology Press. London

Conners F., Carr M., Willis S. (1998) Is the phonologicical loop responsible for Intelligence - Related differences in forward digit span?

American Journal on Mental Retardation 103, 1-11.

Culbertson W., Zillmer E. (1998a)

The Tower of London Dx : A standardized approach to assessing executive functioning in children

Archives of Clinical Neuropsychology 13, 285-301.

Culbertson W., Zillmer E. (1998b)

The Construct Validity of the Tower of London Dx as a measure of the executive functioning of ADHD children.

Assessment 5, 215-226.

Cummings J. (1993) Frontal-Sub cortical Circuits and Human Behavior Archives of Neurology_50,873-880.

Curfs L.M.G., Fryns J-P. (1992) Prader-Willi syndrome: a review with special attention to the cognitive and behavioural profile.

Birth Defects: Original article series 28, 99-104.

Curfs L.M.G., Wiegers A.M., Sommers J.R.M., Borghgraef M., Fryns J.P. (1991) Strengths and weaknesses in the cognitive profile of youngsters with Prader-Willi syndrome.

Clinical Genetics 40, 430-434.

Damasio A. (1994) Descartes 'error: emotion and reason and the human brain. Grosset/Putnam. New York.

Damasio A. (1996) The somatic marker hypothesis and the possible functions of the prefrontal cortex.

Philosophical Transactions of the Royal Society of London B. 351, 1413-1420.

De Bellis M.D., Keshavan M.S., Beers S.R., Hall J., Frustaci K., Masalehdan A., Noll J., Boring A.M. (2001) Sex differences in brain maturation during childhood and adolescence.

Cerebral Cortex 11, 552-557. 
D`Esposito M., Aguirre G.K., Zarahn E., Shin R.K., Lease J. (1998) Functional MRI studies of spatial and nonspatial working memory.

Cognitive Brain Research. 7, 1-18.

Denckla M.B. (1996)

A Theory and Model of Executive Function: a Neuropsychological Perspective. In Lyon G.R. and Krasnegor N.A. (eds) Attention, Memory and Executive Function 263-278. Paul Brookes, Baltimore.

Dimitropoulos A., Feurer I.D., Butler M.G., Thompson T. (2001) Emergence of compulsive behavior and tantrums in children with Prader-Willi syndrome.

American Journal on Mental Retardation 106, 39-51.

Donaldson M.D.C. (2002) Natural history of Prader-Willi syndrome

KIGS Biannual Report 20, 11-21.

Drevets. W.C (2001)

Neuroimaging and neuropathological studies of depression: implications for the cognitive-emotional features of mood disorders.

Current Opinion in Neurobiology 11, 240-249

Drewe E.A. (1975) Go-no go learning after frontal lobe lesions in humans.

Cortex 11, 8-16.

Duncan J., Owen A. (2000) Common regions of the human frontal lobe recruited by diverse cognitive demands.

Trends in Neuroscience 23, 475-483.

Dykens E.M. (1995) Measuring Behavioral Phenotypes: Provocations from the "New Genetics".

American Journal on Mental Retardation 99 522-532.

Dykens E.M. (2002) Are jigsaw puzzle skills “spared” in persons with Prader-Willi syndrome?

Journal of Child Psychology and Psychiatry 43, 343-352.

Dykens E.M. (2004) Maladaptive and compulsive behavior in Prader-Willi syndrome: new insights from older adults.

American Journal on Mental Retardation 109, 142-153.

Dykens E.M., Cassidy S.B. (1995) Correlates of maladaptive behavior in children and adults with Prader-Willi syndrome.

American Journal of Medical Genetics 60, 546-549.

Dykens E.M., Cassidy S.B. (1996) Prader-Willi syndrome: Genetic, Behavioral and Treatment issues.

Child and Adolescent Psychiatric Clinics of North America 5, 913-927. 
Dykens E.M., Cassidy S.B., and King B.H. (1999) Maladaptive Behavior differences in Prader-Willi syndrome due to paternal deletion versus maternal uniparental disomy.

American Journal on Mental Retardation 104, 67-77.

Dykens E.M., Hodapp R., Walsh K., Nash L. (1992a) Adaptive and maladaptive behavior in Prader-Willi syndrome.

Journal of the American Academy of Child and Adolescents Psychiatry 31, 11311135.

Dykens E.M., Hodapp R., Walsh K.,Nash L (1992b) Profiles, Correlates and Trajectories of Intelligence in Prader- Willi syndrome.

Journal of American Academy of Child and Adolescent Psychiatry 31,1125-1130.

Dykens E.M., Leckman J., Cassidy S.B. (1996) Obsessions and Compulsions in Prader-Willi Syndrome

Journal of Child Psychology and Psychiatry 37, 995-1002.

Dykens E.M. and Kasari C. (1997) Maladaptive behaviour in children with PraderWilli syndrome, Down syndrome and Non-specific mental retardation American Journal on Mental Retardation 102, 228- 237.

Dykens E.M., Rosner B. (1999) Refining Behavioural Phenotypes: PersonalityMotivation in Williams and Prader-Willi syndromes.

American Journal on Mental Retardation 104, 158-169.

Einfield S.,Smith A.,Durvasula S.,Florio T.,Tonge B. (1999)

Behavior and Emotional disturbance in Prader-Willi syndrome

American Journal of Medical Genetics 82, 123-127

Einfeld S., Tonge B. (1994) Manual for the Developmental Behaviour Checklist (primary carer version). Monash University Centre for Developmental Psychiatry, Melbourne, Australia.

Einfeld S., Tonge B. (1995) The Developmental Behaviour Checklist: the development and validation of an instrument to assess behavioural and emotional disturbance in children and adolescents with mental retardation.

Journal of Autism and Developmental Disorders 25, 81-104.

Elliot. R, Dolan. R.J, Frith.C.D (2000)

Dissociable functions in the medial and lateral orbitofrontal cortex: evidence from human neuroimaging studies.

Cerebral Cortex 10, 308-317 
Eslinger P.J. (1996) Conceptualizing, describing and measuring components of executive function: A summary. In Lyon G.R., Krasnegor N.A. (eds). Attention, memory and executive function. 367-396. Brookes, Baltimore.

Evans J., Chua S., McKenna P.J., and Wilson B.A. (1997)

Assessment of the dysexecutive syndrome in schizophrenia.

Psychological Medicine 27, 635-646.

Gabel S., Tarter R., Gavaler J., Golden W., Hegedus A., Maier B. (1986)

Neuropsychological capacity of Prader-Willi Children: General and Specific Aspects of Impairment.

Applied Research in Mental Retardation_101, 195-205.

Garner C., Callias M., Turk J. (1999) Executive function and theory of mind performance in boys with fragile-X syndrome

Journal of Intellectual Disability Research 43, 466-474.

Gathercole S., Willis C., Baddeley A.D., Emslie H. (1992) Phonological memory and vocabulary development during the early school years: a longitudinal study.

Developmental Psychology 28, 887-898.

Gerstadt C., Hong Y.J., Diamond A. (1994) The relationship between cognition and action: performance of children $3.5-7$ years old on a Stroop-like day-night test

Cognition 53, $129-153$.

Grant D., Berg E. (1948) Behavioral analysis of degree of reinforcement and ease of shifting to new responses in Weigl - type card sorting problems.

Journal of Experimental Psychology 38, 404-411.

Heaton R.K. (1981) Wisconsin Card Sorting Test Manual. Psychological assessment resources Inc. Odessa FL.

Henry L., MacLean M. (2002) Working memory performance in children with and without intellectual disabilities.

American Journal on Mental Retardation 107, 421-432.

Hodapp R (1997) Direct and indirect behavioral effects of different genetic disorders of mental retardation.

American Journal on Mental retardation 102, 67-79.

Hodapp R., Dykens E.M. (2001) Strengthening behavioral research on genetic mental retardation syndromes.

American Journal on Mental Retardation 106, 4-15. 
Holland A.J.,Treasure J., Coskeran P., Dallow J. (1995) Characteristics of the eating disorder in Prader-Willi syndrome: implications for treatment.

Journal of Intellectual Disability Research_39, 373-381.

Holland A.J., Whittington J.E., Butler J., Webb T., Boer H. and Clarke D. (2003)

Behavioural Phenotypes associated with specific genetic disorders: evidence from a population based study of people with Prader-Willi syndrome

Psychological Medicine 33, 141-153.

Holm V., Cassidy S.B., Butler M., Hanchett J., Greenswag L., Whitman B., Greenberg F. (1993) Prader-Willi syndrome: Consensus diagnostic criteria.

Paediatrics 91, 398-402.

Holsen L., Thompson T. (2004) Compulsive behavior and eye blink in Prader-Willi syndrome: Neurochemical implications.

American Journal on Mental Retardation 109, 197-207.

Hughes C (1996) Control of Action and Thought: Normal development and dysfunction in Autism: A research note

Journal of Child Psychology and Psychiatry 37, 229 -236.

Hughes C., Graham A. (2002)

Measuring Executive Functions in Childhood: Problems and Solutions?

Child and Adolescent mental Health 7, 131-142.

Hulme C., MacKenzie S. (1992) Working memory and severe learning disabilities.

Erlbaum. Hillsdale NJ.

Hutton S., Puri B., Duncan L., Robbins T., Barnes T., Joyce E. (1998) Executive function in first episode schizophrenia.

Psychological Medicine 28, 463-473.

Jarrold C., Baddeley A.D., Hughes A. (2000) Verbal short-term memory deficits in Down syndrome: A consequence of problems in rehearsal.

Journal of Child Psychology and Psychiatry 40,233-244.

Kerr A., Zelazo P. (2004) Development of "hot" executive function: The children's gambling task.

Brain and Cognition. 55, 148-157.

Kringelbach M., Rolls E.T. (2004) The functional neuroanatomy of the human orbitofrontal cortex: evidence from neuroimaging and neuropsychology.

Progress in Neurobiology 72, 341-372.

Laws G., Gunn D. (2004) Phonological memory as a predictor of language comprehension in Down syndrome: A five-year follow up study.

Journal of Child Psychology and Psychiatry 45, 326-337. 
Levin H., Culhane K., Hartman J. Evankovich K., Mattson A., Harward H., Ringholz.,G. Ewing-Cobbs L., Fletcher J.M. (1991)

Developmental changes in performance on tests of purported frontal lobe functioning Developmental Neuropsychology 7, 377-395.

Luciana M. (2003) Computerized assessment of neuropsychological function in children: clinical and research applications of the Cambridge Neuropsychological testing Automated battery (CANTAB).

Journal of Child Psychology and Psychiatry 44, 649-664.

Lucignani G., Panzacchi A., Bosio L., Moresco R.M., Ravasi L., Coppa I., Chiumello G., Frey K., Koeppe R., Fazio F. (2004) GABAa receptor abnormalities in Prader-Willi syndrome assessed with positron emission tomography and $\{11$

C) flumazenil.

NeuroImage 22, 22-28.

Manly T., Robertson I., Anderson V., Nimmo-Smith I. (1999) The test of everyday attention for children: TEA - Ch. Thames Valley Test Co. Bury-St-Edmunds.

Marshburn E., Aman M. (1992). Factor validity and norms for the Aberrant Behavior Checklist in a community sample of children with mental retardation.

Journal of Autism and Developmental Disorders 22, 357-373.

McEvoy R. Rogers S., Pennington B. (1993) Executive function and social communication deficits in young autistic children Journal of Child Psychology and Psychiatry 34,563-578.

Milner B. (1964) Some effects of frontal lobe lobectomy in man. In Warren J., Akert $\mathrm{K}$. (eds) The frontal granular cortex and behavior. McGraw - Hill, New York.

Miyake A., Friedman NP., Emerson M.J., Witzki A.H., Howerter A. (2000) The unity and diversity of executive functions and their contributions to complex "frontal lobe" tasks: a latent variable analysis.

Cognitive Psychology 41, 49-100.

Munir F., Cornish K., Wilding J. (2000) A neuropsychological profile of attention deficits in young males with fragile $\mathrm{X}$ syndrome.

Neuropsychologia 38, 1261-1270.

Newcombe F. (1969). Missile wounds of the brain: a study of psychological deficits. Oxford UP, Oxford.

Norman D.A., Shallice T. (1980). Attention to action: Willed and automatic control of behaviour. Centre for Human Information Processing (Technical Report no 99). (Reprinted in revised form in Davidson R., Schwartz G., Shapiro D. (eds). (1986) Consciousness and self-regulation (vol.4) 1-18. Plenum Press, New York. 
Numminen H., Lehto J.E., Ruoppila I. (2001) Tower of Hanoi and working memory in adult persons with intellectual disability.

Research in Developmental Disabilities. 22, 373-387.

Oosterlaan J., Sergeant J.A. (1998) Response inhibition in ADHD,CD, comorbid $\mathrm{ADHD}+\mathrm{CD}$, anxious and normal children: A meta-analysis of studies with the stop task.

Journal of Child Psychology and Psychiatry 39, 411-426.

Overman W. (2004) Sex differences in early childhood, adolescence and adulthood on cognitive tasks that rely on orbital prefrontal cortex.

Brain and Cognition 55, 134-147.

Owen A.M., Lee A.C.H., Williams E.J. (2000) Dissociating aspects of verbal working memory within the human frontal lobe: Further evidence for a "process specific" model of lateral frontal organisation.

Psychobiology 28, 146-155.

Paulesu. E, Frith. C.D, Frackowiak. R.S.J (1993)

The neural correlates of the verbal component of working memory

Nature 362, 342-343

Paus T., Zijdenbos A., Worsley K., Collins L.D., Blumenthal J., Giedd J.N., Rapoport J.L.,Evans A.C. (1999) Structural maturation of neural pathways in children and adolescents : In vivo study.

Science 283, 1908-1911.

Prader A., Labhart., Willi H. (1956)

Ein Syndrom von Adipositas, Kleinwuchs,Kryptorchismus und Oligophrenie nach myatonieartigen Zustand im Neugeborenenalter.

Schweizerische Medizinische Wochenschrift 86, 1260-1261

Pennington B.F. (2002)

The Development of Psychopathology: Nature and Nurture. Guildford Press, New York.

Pennington B.F., Bennetto L., McAleer O., Roberts R. (1996) Executive functions and working memory: theoretical and measurement issues. In Lyon G.R., Krasnegor N.A. (eds). Attention, Memory and Executive Function. 327-348. Brookes, Baltimore.

Pennington B.F., Ozonoff S. (1996) Executive functions and developmental psychopathology.

Journal of Child Psychology and Psychiatry 37, 51-88. 
Petrides M. (1998) Specialised systems of the processing of mnemonic information within the primate frontal cortex. In Roberts A.C., Robbins T.W., Weiskrantz L. (eds). The Prefrontal Cortex: Executive and cognitive functions. 103116. Oxford University Press, Oxford.

Petrides M., Milner B. (1982) Deficits on subject ordered tasks after frontal and temporal lobe lesions in man.

Neuropsychologia 20, 249-262.

Phillips L. (1997) Do “frontal tests” measure executive function?: Issues of assessment and evidence from fluency tests. In Rabbitt P. (ed|) Methodology of frontal and executive function. 191-214. Psychology Press, Hove.

Phillips L., Wynn V., Gilhooly K., Della Sala S., Logie R. (1999) The role of memory in the Tower of London Task.

Memory 7, 209-231.

Porteus S.D.(1965) The Porteus Maze Test.Psychological Corporation.New York.

Richdale A., Cotton S., Hibbit K. (1999) Sleep and behavioural disturbance in Prader-Willi syndrome: a questionnaire study.

Journal of Intellectual Disability Research 43, 380-392.

Robbins T.W. (1998) Dissociating executive functions of the prefrontal cortex. In Roberts A.C., Robbins T.W., Weiskrantz L. (eds). The Prefrontal Cortex: Executive and cognitive functions. 117-130. Oxford University Press, Oxford..

Rolls E.T. (1999) The Brain and Emotion.

Oxford University Press. Oxford

Roof E., Stone W.,MacLean W., Feurer I.,Thompson. T., Butler M.G.(2000)

Intellectual characteristics of Prader-Willi syndrome: comparison of genetic subtypes

Journal of Intellectual Disability Research 44, 25-30.

Rosenquist C., Conners F., Roskos-Ewoldsen B. (2003) Phonological and visuospatial working memory in individuals with intellectual disability.

American Journal on Mental Retardation 108, 403-413.

Sahakian B., Owen A. (1992) Computerized assessment in neuropsychiatry using CANTAB.

Journal of the Royal Society of Medicine. 85, 399-402.

Salmon E., Van der Linden M., Collette F., Delfiore G., Maquet P., Degueldre C., Luxen A., Franck G. (1996) Regional brain activity during working memory tasks. Brain 19, 1617-1625. 
Sarimski K. (1997) Behavioural phenotypes and family stress in three mental retardation syndromes

European Child and Adolescent Psychiatry 6, 26-31.

Savage. C.R, Deckersbach. T, Heckers. S, Wagner. A.D, Schacter.D.L, Alpert. N.M, (2001) Prefrontal regions supporting spontaneous and directed application of verbal learning strategies, evidenced from PET.

Brain 124, 219-231

Shallice, T. (1982) Specific impairments of planning

Philosophical Transactions of the Royal Society of London B 298, 199-209.

Sparrow S., Balla D., Cicchetti D. (1984) Vineland Adaptive Behavior Scales.

NFER- Nelson, Windsor.

State M., Dykens E.M., Rosner B., Martin A., King B. (1999) Obsessive compulsive symptoms in Prader-Willi and "Prader-Willi like" patients.

Journal of the American academy of Child and Adolescent Psychiatry 38, 329-334.

Stauder J.E.A., Brinkman M.J.R., Curfs L.P.G. (2002) Multi-modal P3 deflation of event related brain activity in Prader-Willi syndrome.

Neuroscience Letters 327, 99-102.

Steinhausen H., Eiholzer U., Hauffa B.P., Malin Z. (2004) Behavioural and emotional disturbances in people with Prader-Willi syndrome.

Journal of Intellectual Disability Research 48, 47-52.

Stroop J.R. (1935) Studies of interference in serial verbal reactions.

Journal of Experimental Psychology 18, 643-6662.

Swaab D., Purba J., Hofman M. (1995) Alterations in the hypothalamic paraventricular nucleus and its oxytocin neurons (putative satiety cells) in PraderWilli syndrome: a study of five cases.

Journal of Clinical Endocrinology and Metabolism. 80, 573-579.

Tamm L., Menon V., Reiss A.I. (2002) Maturation of brain function associated with response inhibition.

Journal of the American Academy of Child and Adolescent Psychiatry 41, 12311238.

Temple C., Carney R., Mullarkey S. (1996) Frontal lobe function and executive skills in children with Turner's syndrome Developmental Neuropsychology 12, 342-363. 
Thatcher R.W. (1991) Maturation of the human frontal lobes: Physiological evidence for staging.

Developmental Neuropsychology 7, 397-419.

Thurstone L. (1938). Primary mental abilities. University of Chicago Press, Chicago.

Tsukiura T., Fujii T., Takahashi T., Xiao R., Inase M., Iijima T., Yamadori A., Okuda J. (2001) Neuroanatomical discrimination between manipulating and maintaining processes involved in verbal working memory: a functional MRI study. Cognitive Brain Research 11, 13-21.

Verhoeven W., Curfs L., Tuinier S. (1998) Prader-Willi syndrome and cycloid psychoses.

Journal of Intellectual Disability Research 42,455-462.

Vicari S., Carlesimo G.A., Caltagirone C. (1995) Short-term memory in people with intellectual disabilities and Down's syndrome.

Journal of Intellectual Disability Research 39, 532-537.

Vicari S., Marotta L., Carlesimo G.A. (2004) Verbal short-term memory in Down's syndrome: An articulatory loop deficit?

Journal of Intellectual Disability Research 48, 80-92.

Volkow. N.D, Fowler. J.S, (2000)

Addiction, a disease of compulsion and drive: involvement of the orbitofrontal cortex.

Cerebral Cortex 10, 318-325

Waters J. (1999)

Prader-Willi syndrome: A Practical Guide. David Fulton, London

Webb T., Whittington J.E., Clarke D.J., Boer H., Butler J., Holland A.J. (2002) A study of the influence of different genotypes on the physical and behavioral phenotypes of children and adults ascertained clinically as having PWS.

Clinical Genetics 62, 273-281.

Wechsler D. (1976) Wechsler Intelligence Scale for Children Revised.

Psychological Corporation. London

Wechsler D. (1981) Wechsler Adult Intelligence Scale Revised. Psychological

Corporation, London.

Welsh M., Pennington B.F. (1998) Assessing frontal lobe functioning in children: Views from developmental psychology.

Developmental Neuropsychology 4, 199-230. 
Whittington J.E., Holland A.J., Webb T., Butler J.V.,Clarke D., Boer H. (2001) Population prevalence and estimated birth incidence and mortality rate for people with Prader-Willi syndrome in one UK Health Region Journal of Medical Genetics 38, 792-798.

Whittington J.E., Holland A.J., Webb T., Butler J.V., Clarke D. J., Boer H. (2004a) Cognitive Abilities and genotype in a population based sample of people with Prader-Willi Syndrome.

Journal of Intellectual Disability Research 48, 172-187.

Whittington J.E., Holland A.J., Webb T., Butler J.V., Clarke D.J., Boer H. (2004b) Academic underachievement in Prader-Willi syndrome Journal of Intellectual Disability Research 48, 188-200.

Wigren M., Hansen S. (2003) Rituals and compulsivity in Prader-Willi syndrome: profile and stability.

Journal of Intellectual Disability Research 47, 428-438.

Wilding J. (1997) Wilding attention tests. University of London Department of Psychology, Royal Holloway.

Wilding J., Cornish K., Munir F. (2002) Further delineation of the executive deficit in males with fragile-X syndrome.

Neuropsychologia 40, 1343-1349.

Wilson B., Alderman N., Burgess P., Emslie H., Evans.J.(1996) Behavioural Assessment of the Dysexecutive Syndrome (BADS)

Thames Valley Test Company. Suffolk.U.K

Wilson B., Evans J., Alderman N., Burgess P., Emslie H. (1997) Behavioural assessment of the dysexecutive syndrome. In Rabbitt P. (ed|) Methodology of frontal and executive function. 239-243. Psychology Press, Hove..

Yoshii A., Krishnamoorthy K.S., Grant E.P. (2002) Abnormal cortical development shown by 3D MRI in Prader-Willi syndrome.

Neurology 59, 644-645.

Zelazo P. (1998) Language, levels of consciousness, and the development of intentional action. IN Zelazo P. Astington J., Olson R.D. (eds) Developing Theories of intention: Social understanding and self control .95-118. Erlbaum, Hillsdale NJ.

Zelazo P. (2000 Self reflection and the development of consciously controlled processing. In Mitchell P. Riggs K. (eds) Children 's reasoning and the mind. 169189.Psychology Press, Hove. 
Zelazo P., Muller U., (2002) Executive function in typical and atypical development. In Goswami U. (ed) Handbook of childhood cognitive development. 445-469.

Blackwell. Oxford. 


\section{Appendix A}

Examples of consent and information letters.

NB All identifying information has been deleted.

1. Information letter for parents and carers of participants with PWS.

2. Consent letter for parents and carers of participants with PWS.

3. Information letter for participants with PWS.

4. Consent letter for participants with PWS.

5. Information letter for parents and carers of comparison group participants.

6. Consent letter for parents and carers of comparison group participants.

7. Information letter for comparison group participants.

8. Consent letter for comparison group participants.

9. Information letter to G.P.

10. Consent letter to G.P. 


\section{$\underline{\text { 1.Information letter for parents/carers }}$}

Investigation of Executive Function in Adults with Prader-Willi Syndrome: A pilot project.

Mr Robert Walley, Department of Clinical Psychology, Address deleted.

As parents and carers of an adult with Prader-Willi Syndrome, I would very much value your help in a project I would like to carry out which I hope will further our understanding of Prader-Willi Syndrome.

\section{Why I want to do this research}

A number of studies have shown that many young people and adults with PraderWilli Syndrome have emotional and behavioural problems in addition to concerns regarding food. Emotional and behaviour problems that have been observed include temper tantrums, self-injury, impulsive behaviour, and rapid changes in mood, inactivity and repetitive talking. It has also been observed that obsessive-compulsive behaviours are commonly seen in Prader-Willi Syndrome, and that many adults like things to be in order, and may be upset by changes in routine.

Although much research has been carried out on the genetics of Prader-Willi Syndrome, there has not been a great deal of research investigating why people with Prader-Willi Syndrome, show these behaviour and emotional problems. Trying to find out why these problems occur may not only help parents and carers understand more about the syndrome, but also may lead to improvements in ways of managing the behaviours concerned. Such work could also help adults with Prader-Willi Syndrome in the areas of education and employment.

I am particularly interested in investigating the abilities of planning, organising, and self-monitoring of behaviour, which may underlay some of the behavioural problems seen in Prader-Willi Syndrome. These abilities are called "executive function" because of their importance in directing our behaviour. 


\section{What I would like to do}

In order to do this, I would ask your son or daughter or the person you care for to attend the

Hospital (details deleted), to undertake a number of psychological tests. It is hoped that it will not take longer than about 2-3 hours to complete all the tests, but if your son or daughter should find it hard going, I could arrange to see them at a further time and/or stop for a break.

If your son or daughter attended at the hospital, and was unwilling to become involved in the project at that time, or after testing has begun, I would not put them under any pressure to remain.

Adults participating in the project and parents/carers accompanying them would be given the necessary travelling expenses.

It should be emphasized that all the tests involved are psychological, and there will be no need to take any blood or tissue samples, or undertake any medical tests in connection with this project. I will also be asking parents/carers to complete 2 questionnaires. One questionnaire relates to general problems of behaviour, which has been used in other studies involving adults with Prader-Willi Syndrome. The second questionnaire relates to particular problems associated with "executive function" difficulties.

\section{Confidentiality}

Although new knowledge from the research may be made known to others by means of published papers, it will not be possible to identify the individuals who have taken part in the project.

Before any testing is commenced, I would ask you to sign a consent form giving permission for your son or daughter to take part. You will be able to keep a copy of the consent form. 
I feel that this project has considerable potential in helping us understand much more about the psychology of Prader-Willi Syndrome and I would be very grateful for your support and backing. If you have any questions about the project please do not hesitate to contact me.

\section{Contact details}

Mr Robert Walley, Chartered Clinical Psychologist, Department of Psychology, Address deleted. 


\section{Consent letter for parents/carers of participants with PWS}

\section{Investigation of executive function in adults with Prader-Willi Syndrome}

I have read the information sheet relating to the above project, and am willing to give consent for my son/daughter to take part in the project. I understand that if after commencing testing my son/daughter wishes to withdraw from the project they will be free to do so at any time, and will not be put under any obligation to remain. I understand however that should they feel tired during a testing session, and wish to return at another time they will be given that opportunity. I understand that my son/daughter's participation in the project is confidential, although my son/daughter's GP has also been informed that they are taking part.

Name of participant:

Age:

Sex:

\section{Address:}

Signature of parent/carer: 


\section{$\underline{\text { 3.Information letter for participants with PWS }}$}

Investigation of Executive Function in Adults with Prader-Willi Syndrome: A pilot project.

Mr Robert Walley, Department of Clinical Psychology, Address deleted

I would be very grateful if you would help me with a project, which I hope will help us understand more about Prader-Willi Syndrome.

I hope that the project will tell us more about the things that people with Prader-Willi Syndrome are good at doing, as well as about things that people with Prader-Willi Syndrome have some difficulties with.

I would particularly like to find out how good adults with Prader-Willi Syndrome are at being able to plan things, and also how good they are at being able to change from one activity to another.

To do this research I would like you to come to the hospital (details deleted), where I will ask you to do a number of psychological tests. There will be no need to have any medical tests done if you take part in this project.

If you decide you would like to take part, you would be able to bring a parent or friend with you. Before testing begins, I will explain to you what the tests are about, and you can ask any questions about them if you want to.

The tests themselves are quite short, but altogether they will take about 2 or 3 hours to do. If you become tired during this time, it will be possible to take some breaks, or to arrange for an appointment to finish on another day. If you want to stop the tests at any time that will be O.K. We will just stop the testing and you can go home. 
When I have finished the project, I would hope to tell other people about it, but I would not use anybody's name, and nobody would know who had taken part. I would also tell your family doctor that you were willing to take part in this research. 


\section{Consent letter for Participants with PWS}

\section{Investigation of executive function in adults with Prader-Willi Syndrome}

I have been given information about a project that $\mathrm{Mr}$ Walley wants to do with adults who have Prader-Willi Syndrome. I understand that this project is being done to help us learn more about Prader-Willi Syndrome. I am happy to take part.

I understand that if I change my mind about taking part in the project, that will be $\mathrm{OK}$ and no one will try to force me to do the tests. If I become tired it will be possible to stop and come along on a different day. I understand that the information obtained in this project will be kept private, although my doctor has been told that I am taking part.

Signature of participant:

Name of participant:

Age:

Sex:

Address: 


\section{Information letter for Parents/Carers of comparison group participants}

Investigation of Executive Function in Adults with Prader-Willi Syndrome: A pilot project.

Mr Robert Walley, Department of Clinical Psychology, Address deleted.

\section{Background to the project}

I would be very grateful, if you could give consideration, for your son or daughter to help me in a project investigating executive function in adults with Prader-Willi Syndrome. Prader-Willi Syndrome, as you may know, is commonly associated with eating difficulties. Additionally however many people with Prader-Willi Syndrome show emotional and behavioural problems that include temper tantrums, self-injury, impulsive behaviour, rapid changes in mood, inactivity and repetitive talking.

Executive function abilities help us to plan, organise, and self monitor our behaviour. They also help us switch from one topic or activity to another, and enable us to know when an event occurred. I believe that problems in executive function, in PraderWilli Syndrome, may be related to some of the emotional and behavioural problems seen in that syndrome.

\section{What I would like to do}

In order to investigate this, I need to carry out a number of psychological tests with adults who have Prader-Willi Syndrome, and also with adults who do not. The adults who do not have Prader-Willi Syndrome who help us in this project are called comparison participants, because we want to see if their scores on tests differ from the scores of participants who do have Prader-Willi Syndrome. In order to ensure that I am seeing true differences it is important that comparison participants are as similar as possible to the participants who have Prader-Willi Syndrome. 
Testing will take place at the hospital (details deleted), your home or any other convenient place.

It must be emphasized that all the tests will be psychological in nature and there will be no requirement to take samples of blood etc.

It is hoped that it will not take longer than about 2 or 3 hours to complete all the tests but if your son or daughter should find it hard going I would arrange to see them for a further appointment, and/or stop for a break. If your son or daughter attended the hospital and was unwilling to become involved in the project at that time, I would not put them under any pressure to remain but would again offer a further appointment if desired. If your son/daughter wish to stop at any time testing will be abandoned.

Adults participating in the project and parents/carers accompanying them would be given the necessary travelling expenses.

I will also be asking parents/carers to complete 2 questionnaires. One questionnaire relates to general problem behaviour, which has been used in a number of studies involving adults with Prader-Willi Syndrome. The second questionnaire relates to particular problems associated with 'executive function difficulties'.

\section{Confidentiality}

Although new knowledge from research may be made known to others by means of published papers it will not be possible to identify the individuals who have taken part in the project.

Before any testing is commenced I would ask you to sign a consent form giving permission for your son/daughter to take part. You will be able to keep a copy of the consent form. I would very much value your help and support in this project which I hope will further our understanding of Prader-Willi Syndrome. If you have any questions about the project please do not hesitate to contact me. 


\section{Contact details}

Mr Robert Walley, Chartered Clinical Psychologist, Department of Psychology, Address deleted. 


\section{Consent letter for parents/carers of comparison group participants}

\section{Investigation of executive function in adults with Prader-Willi Syndrome}

I have read the information sheet relating to the above project, and am willing to give consent for my son/daughter to take part in the project. I understand that if after commencing testing my son/daughter wishes to withdraw from the project they will be free to do so at any time, and will not be put under any obligation to remain. I understand however that should they feel tired during a testing session, and wish to return at another time they will be given that opportunity. I understand that my son/daughter's participation in the project is confidential, although my son/daughter's GP has also been informed that they are taking part.

Name of participant:

Age:

Sex:

\section{Address:}

Signature of parent/carer: 


\section{Information letter for comparison group participants}

Investigation of Executive Function in Adults with Prader-Willi Syndrome: A pilot project.

Mr Robert Walley, Department of Clinical Psychology, Address deleted.

I would be very grateful if you would help me with a project, which I hope would help us understand more about Prader-Willi Syndrome.

I hope that the project will tell us more about the things that people with Prader-Willi Syndrome are good at doing, as well as about things that people with Prader-Willi Syndrome have some difficulties with.

I know that you do not have Prader-Willi Syndrome, but to do this project, it is important to be able to see if a group of people who do not have Prader-Willi Syndrome, do things differently, from people that do have Prader-Willi Syndrome.

I would particularly like to find out how good adults with Prader-Willi Syndrome are, at being able to plan things, and also how good they are at being able to change from one activity to another.

To do this research I would like you to come to the hospital (details deleted), where I will ask you to do a number of psychological tests. If it would be more convenient I could see you at home. There will be no need to have any medical tests done if you take part in this project.

If you decide you would like to take part, you would be able to bring a parent or friend with you. Before testing begins, I will explain to you what the tests are about, and you can ask any questions about them if you want to. 
The tests themselves are quite short, but altogether they would take about 2 or 3 hours to do. If you become tired during that time, it would be possible to take some breaks, or to arrange for an appointment to finish on another day.

If you want to stop the tests at any time that will be O.K. We will just stop the testing and you could go home.

When I have finished the research, I would hope to tell other people about it, but I would not use anybody's name, and nobody would know who had taken part. I would also tell your family doctor, that you were willing to take part in this research. 


\section{$\underline{\text { 8.Consent letter for Comparison group participants }}$}

\section{Investigation of executive function in adults with Prader-Willi Syndrome}

I have been given information about a project that $\mathrm{Mr}$ Walley wants to do with adults who have Prader-Willi Syndrome. I understand that this project is being done to help us learn more about Prader-Willi Syndrome. I am happy to take part as a member of a comparison group.

I understand that if I change my mind about taking part in the project, that will be $\mathrm{OK}$ and no one will try to force me to do the tests. If I become tired it will be possible to stop and come along on a different day. I understand that the information obtained in this project will be kept private, although my doctor has been told that I am taking part.

Signature of participant:

Name of participant:

Age:

Sex:

Address: 


\section{Information sheet for GP's and other Healthcare Professionals}

\section{Investigation of Executive Function in Adults with Prader-Willi Syndrome : A pilot project.}

Prader-Willi Syndrome occurs in approximately 1 in 15,000 births, and usually results in mild to moderate learning disability. About $70 \%$ of Prader-Willi Syndrome cases are caused by a paternally derived deletion on chromosome 15; the remaining $30 \%$ are generally caused by maternal uni-parental disomy or when both members of the chromosome 15 pair are derived from the mother.

Prader-Willi Syndrome is characterized and best known by its food related problems i.e. hyperphagia, food pre-occupation, and foraging. Individuals with Prader-Willi Syndrome have a high risk of developing obesity. Excessive daytime sleepiness, and sleep onset rapid eye movements are also commonly observed traits.

In addition to these problems, many people with Prader-Willi Syndrome show significant difficulties in the area of behaviour and emotional functioning. Problems such as temper tantrums, stubbornness, emotional lability, impulsiveness, argumentativeness, depression, anxiety, and repetitive behaviours such as skin picking, are commonly seen. Obsessive-compulsive behaviour beyond the preoccupation with food has also been observed in this population, suggesting increased risks of full-blown obsessive disorder. People with Prader-Willi Syndrome also appear to be vulnerable to developing psychoses. These behavioural and emotional problems, beginning in childhood, can be particularly challenging in adolescence and early adulthood, and put considerable strain on family dynamics.

A few studies have investigated cognitive functioning in Prader-Willi Syndrome, but none have specifically looked at possible neuropsychological correlates for the behaviours mentioned above. These studies indicate that people with people with Prader-Willi Syndrome, have short-term auditory verbal and visual memory difficulties, and that their sequential processing abilities (verbal reasoning) are poorer 
than their simultaneous processing ability (ability to process visual/perceptual information).

The adult and developmental literature suggests that damage to the pre-frontal cortex can result in a similar behavioural and emotional problems to those seen in PraderWilli Syndrome The underlying neuropsychological correlates are believed to be deficits in executive function, i.e. initiation of behaviour, planning, self-monitoring inhibitions of a pre-potent response, and working memory.

The purpose of the present project is to ascertain if some of the behavioural and emotional problems seen in Prader-Willi Syndrome have a neuropsychological correlate in terms of abilities/deficits in executive function. This will be investigated by comparing the performance of a group of adults with Prader-Willi Syndrome on a small number of executive function tests, with adults with non-specific learning disability, matched for age gender and verbal abilities. Parents/carers will also be asked to complete 2 questionnaires, one relating to general behaviour, and another one relating specifically to problems in executive function. Adult participants will be drawn from Dr X's (name deleted) clinic at the hospital (details deleted), and $\mathrm{Mr}$ Robert Walley, Chartered Clinical Psychologist, will carry out the testing. The consent of parents/carers will be obtained for each participant recruited from the clinic. All data collected will be treated confidentially and for the purpose of any published papers will be collated so that it will not be possible to identify individuals. 


\section{Letter to GP indicating participation of a patient}

\section{Investigation of executive function in adults with Prader-Willi Syndrome}

Dear Dr

Re:

The parents/carers of the above named person, whom I understand is one of your patients, have given their consent to take part in a project investigating executive function in adults with Prader-Willi Syndrome. A brief information sheet is enclosed. Please do not hesitate to contact me if you require further information.

Yours sincerely

Mr Robert Walley

Chartered Clinical Psychologist 


\section{$\underline{\text { Appendix B }}$}

An Investigation of executive function abilities in adults with Prader-Willi syndrome.

R.M.Walley and M.D.C Donaldson (2005)

Journal of Intellectual Disability Research 49 pp 613-625 


\title{
An investigation of executive function abilities in adults with Prader-Willi syndrome
}

\author{
R. M. Walley' \& M. D. C. Donaldson ${ }^{2}$ \\ I Department of Psychology, Edenhall Hospital, Musselburgh, East Lothian, UK \\ 2 Department of Child Health, Royal Hospital for Sick Children, Yorkhill, Glasgow, UK
}

\begin{abstract}
Background Prader-Willi syndrome (PWS) is a genetic disorder caused by the absence of expression of maternally imprinted genes on the long arm of chromosome 15 (I5q II-13). There are two main genetic sub-types: (I) deletion, caused by the absence of paternally derived genetic material; and (2) uniparental disomy (UPD), where two copies of maternally derived chromosome 15 are present. In addition to generally mild/borderline intellectual disability (ID) and the almost universal feature of hyperphagia, PWS is associated with high rates of behaviour problems including temper tantrums, compulsive behaviour, perseverative speech, skin picking and rigid thinking. The present study seeks to explore whether these behaviours are associated with relative deficits in executive function (EF), which comprises the set of non-automatic processes utilized by an individual when faced with a novel situation.

Methods Eighteen adult participants with a clinical diagnosis of PWS (12 with deletion sub-type, 6 with UPD) were recruited from a UK Health Service
\end{abstract}

Correspondence: Robert M Walley, Department of Psychology, Edenhall Hospital, Musselburgh, East Lothian, UK (e-mail: Robert.Walley@lpct.scot.nhs.uk).
PWS clinic, and compared with 15 participants of similar age and verbal ability on a series of EF tasks and also Digit Span Forwards. An informant completed two ratings of behaviour, the Aberrant Behavior Checklist $(\mathrm{ABC})$ and the Dysexecutive Questionnaire (DEX).

Results The PWS group had significantly higher scores on the ABC but not on the DEX. There were no significant differences between the whole PWS group and the comparison group on any of the EF tasks. The deletion sub-type group was significantly poorer at a non-executive task, Digit Span Forwards. There was an unexpected trend for the deletion subtype group to show more efficient performance on a visuospatial planning task, the Tower of London (TOL), but this trend did not reach significance. Conclusions The lack of relative deficits in EF task performance does not support the hypothesis that EF differences could account for the high levels of behaviour problems found in PWS. Applying the Baddeley and Hitch model of working memory it is suggested that the PWS group have a relatively intact central executive and visuospatial sketchpad but a relative impairment in the phonological loop, perhaps relating to the capacity of the phonological store. This latter finding seems to be particularly salient for those with a deletion. As differences in EF ability were not found, it is suggested that a region of the brain 
R. M. Walley \& M. D. C. Donaldson - Executive function abilities in adults with PWS

involved in the modulation of emotion but not particularly with $\mathrm{EF}$, the orbitofrontal cortex (OFC), may be implicated in the behaviour problems reported in PWS.

Keywords executive function, orbitofrontal, Prader-Willi syndrome, working memory

\section{Introduction}

Prader-Willi syndrome (PWS) is a genetic disorder which affects men and women almost equally and is caused by the absence of expression of maternally imprinted genes on the long arm of chromosome is $_{5}$ (I5q II-I3) (Webb et al. 2002). PWS will occur if this part of the paternal chromosome is deleted, or when the paternal chromosome is absent, and two copies of the maternal chromosome 15 are present (uniparental disomy). The deletion sub-type is associated with $70 \%$ and uniparental disomy (UPD) about $25 \%$ of PWS. Around $5 \%$ of PWS is caused by a microdeletion or translocation involving the imprinting centre.

PWS is characterized by infantile hypotonia and feeding problems in the first year of life, followed by hyperphagia in childhood which if not controlled can lead to severe and potentially life-threatening obesity in adulthood. Other characteristics include physical features, endocrine problems affecting growth and sexual development, and developmental delay usually leading to mild or borderline intellectual disability (ID). Holm \& Cassidy (1993) describe further minor and supportive diagnostic criteria for PWS, which include behaviour problems, sleep disturbance and speech and articulation difficulties.

Relative to other genetic syndromes with mild/borderline levels of ID, high rates of behaviour problems are reported in PWS which include temper outbursts, obsessive behaviours, skin picking, perseverative speech, rigid thinking and difficulties in coping with change (Clarke et al. 1996; Dykens \& Kasari 1997; Einfeld et al. 1999). The obsessive behaviours found in PWS tend to be ritualistic behaviours, e.g. objects having to be in a particular order, hoarding, insistence on routines, and repetitiveness of actions and speech, rather than typically obsessive compulsive symptoms such as checking, cleaning, or obsessional thoughts. Whilst temper outbursts and ritualistic behaviours are found in typically developing children, in PWS they continue well beyond the developmental stage at which they would be considered appropriate, and persist into adolescence and adulthood (State et al. 1999; Clarke et al. 2002; Wigren \& Hansen 2003).

Differences in cognitive ability and behaviour between the deletion and UPD sub-types have been observed in that individuals with UPD are reported to have better verbal abilities and fewer behavioural problems (Dykens et al. 1999; Roof et al. 2000; Whittington et al. 2004a). The deletion sub-type have better visuospatial skills and may show splinter skills at jigsaw puzzles (Dykens 2002), although the latter skill may be because of practice effects (Whittington $e t$ al. 2004a). Those with UPD are at high risk of developing an affective psychotic illness in adulthood (Boer et al. 2001).

Individuals with PWS tend to perform better on tasks requiring visual processing skills than tasks that require auditory processing; they are also reported to have poor short-term memories, and deficits in sequential processing. (Curfs et al. I99I; Dykens et al. 1992a).

Despite relatively mild levels of ID, individuals with PWS find it difficult to establish peer group relationships (Waters 1999). Holland et al. (2003) found that their PWS group had a relative strength in the daily living skills domain of the Vineland Adaptive Behaviour scales (Sparrow et al. 1984), but a relative weakness in the socialization domain, confirming similar results obtained by Dykens et al. (I992b). Whittington et al. (2004b) found that academic under-achievement was negatively correlated with scores on the Vineland socialization domain.

Holland et al. (2003) have recently suggested that the compulsive behaviours and temper tantrums seen in PWS might be caused by arrested brain development. It is important, however, to investigate whether the disproportionate levels of behaviour problems reported in PWS are associated with particular difficulties in cognitive processing as that may lead to improved understanding of and treatment for affected individuals. Some behaviours observed in PWS, particularly the temper tantrums, repetitive and perseverative speech, problems in developing peer group relationships, and dislike of change and unpredictability, suggest that individuals with PWS may have executive function (EF) deficits. 
R. M. Walley \& M. D. C. Donaldson - Executive function abilities in adults with PWS

The concept of EF refers to a set of interrelated abilities, which enable an individual to deal successfully with novelty and change in social and non-social settings. EF abilities are utilized to initiate and generate plans, to prioritize actions, and to assist in selfmonitoring and evaluation of behaviour (Pennington \& Ozonoff 1996; Hughes \& Graham 2002). Denckla (I996) suggests that EF should focus on the processes of initiate, sustain, inhibit/stop and shift. EF is associated with activity in the frontal parts of the brain, although it is recognized that a number of brain areas may be involved (Cummings 1993).

An important aspect of EF is working memory, which is the limited capacity memory system responsible for the temporary storage and processing of information while cognitive tasks are being carried out. One theoretical model of working memory is that proposed by Baddeley \& Hitch (1974), Baddeley (I986) and comprises a controlling central executive which is modality free and two slave systems which provide temporary storage and maintenance of information. These are the phonological loop and visuospatial sketchpad. The visuospatial sketchpad stores and maintains visual information and the phonological loop has the same function for verbal material. The phonological loop consists of two sub-systems, a phonological store and an articulatory rehearsal process; in ID the ability to use the articulatory rehearsal process is generally impaired but the visuospatial sketchpad is relatively intact (Rosenquist et al. 2003).

The present study seeks to investigate whether adults with PWS have relative EF deficits, which may contribute to an understanding of their behavioural problems. Specifically it is hypothesized that a group of individuals with PWS compared with a group of individuals of similar language ability will perform more poorly on EF tasks.

\section{Materials and methods}

\section{Participants}

Eighteen individuals with a clinical diagnosis of PWS were recruited from the second author's PWS clinic. In order to control for developmental changes only individuals over the age of 16 were recruited into the study. Eleven of the participants were male and seven were female. All the participants had appropriate genetic testing carried out by a molecular geneticist, and all were found to have a genetic abnormality associated with PWS. Five men and one woman were found to have UPD, and six men and six women were found to have deletions.

The age range of the PWS participants was between $\mathrm{I} 6$ and 49. As language abilities are believed to be related to the ability to do EF tasks (Hughes \& Graham 2002), it was decided to control for verbal abilities using the vocabulary and similarities subtests of the Wechsler Adult Intelligence Scale Revised (WAIS-R, Wechsler I98I). Whilst this does not equate to the verbal IQ score obtained by using all the Wechsler sub-tests, it provides a measure of language ability and is not confounded by attentional factors, which may affect performance on other verbal Wechsler sub-tests, e.g. digit span and arithmetic. Using this short form measure, the verbal IQ of participants ranged from 53 to 93 .

The comparison group were also drawn from a clinical setting and consisted of $\mathrm{I}_{5}$ individuals, seven men and eight women, of similar ages and verbal abilities to the PWS group. All the members of the comparison group had been seen in the Psychology Department in which the first author was employed, and had been referred because of anxiety problems. One member of the comparison group had a genetic syndrome - Down syndrome - the cause of ID for the other participants with ID was not known. The age range of the comparison group was between 18 and 49 , and the range of estimated verbal IQ was between 55 and 9r. Demographic details of the PWS participant and comparison groups are shown in Table $\mathrm{I}$. There were no significant differences in mean verbal IQ and age between the two groups.

\section{Ethical approval}

Ethical approval for the study was obtained from the Hospital Trust in which the second author's multidisciplinary PWS clinic is based, and for the purpose of recruiting the comparison group, also from the first author's former employing Trust.

\section{Measures}

One of the difficulties in assessing EF is that there is no such thing as a pure measure of EF; tasks, which 
R. M. Walley \& M. D. C. Donaldson - Executive function abilities in adults with PWS

Table I Descriptive characteristics of the PWS and comparison groups

\begin{tabular}{llllll}
\hline & $n$ & Mean age (SD) & Male & Female & Verbal IQ (SD) \\
\hline PWS & 18 & $23.5(7.8)$ & 11 & 7 & $73.3(10.6)$ \\
Comparison & 15 & $24.5(8.7)$ & 7 & 8 & $70.6(8.6)$ \\
\hline
\end{tabular}

PWS, Prader-Willi syndrome.

are believed to tap EF, utilize other cognitive abilities (Burgess 1997; Hughes \& Graham 2002).

As general cognitive difficulties will affect performance on EF tasks, a set of measures was chosen which had been used previously in either studies in ID or the developmental literature. The intention was to keep potentially confounding effects of comprehension difficulties to a minimum.

The tasks were believed to require abilities important in studies of EF, initiation of behaviour, planning, set changing, the inhibition of a pre-potent response, and working memory. An important feature of an EF task is that it should be novel and as far as could be ascertained all the EF tasks were novel to the PWS and comparison groups.

The tasks used are listed below and are fully described in the Appendix.

\section{Initiating Behaviour}

Verbal Fluency-Letters FAS and Semantic Categories (Newcombe 1969; Temple et al. 1996).

\section{Planning Ability}

Tower of London (TOL)

A standardised adaptation of the original TOL task (Shallice 1982) was used to assess planning ability, the TOL Drexel Version (Culbertson \& Zillmer 1998).

In order to control for differences in visuospatial ability, participants were also given the Block Design sub-test from the Wechsler Adult Intelligence Scale Revised (WAIS-R).

\section{Set/Rule Changing Ability and Inhibition of a Pre-Potent Response}

Luria Hand Game Adapted by Hughes (1996)

Rule Shift Card Test - Wilson et al. (1996)

Spatial Reversal Task - MacEvoy et al. I993)

Stroop Type Task - Day/Night Version - Gerstadt et al. (1994)

\section{Working Memory}

Self-Ordered Pointing Test - Temple et al. (1996)

Digit span from the WAIS-R (Wechsler I98I)

The experimental tasks were administered in the same order to all participants by the first author. Administration of the tests took approximately one and a half hours.

\section{Behaviour problems}

To measure behaviour problems two questionnaires were given either to a carer, or someone who knew the participant well.

\section{Dysexecutive Questionnaire (DEX) - Wilson et al. (1996)}

This questionnaire is part of the Behavioural Assessment of Dysexecutive Syndrome (BADS) and consists of 20 items constructed to sample a range of difficulties commonly associated with dysexecutive problems, it covers four areas - emotion and personality, motivation, behaviour and cognitive function. Each item is rated on a five-point scale representing problem severity. The total score for the questionnaire was recorded.

\section{Aberrant Behavior Checklist-Community Version (ABC), Aman et al. (1985), Aman \& Singh (1994)}

This is a 58 -item questionnaire, which requires a rating of specified maladaptive behaviour from zero to three representing problem severity over the previous 4 weeks. As well as providing a total score, the Aberrant Behavior Checklist (ABC) yields scores from five factors: (I) irritability and agitation; (2) lethargy and withdrawal; (3) stereotypic behaviour; (4) hyperactivity and non-compliance; and (5) inappropriate speech. The ABC was used in addition to the Dysexecutive Questionnaire (DEX) because of its 
R. M. Walley \& M. D. C. Donaldson - Executive function abilities in adults with PWS

reliability in assessing behaviour problems in people with cognitive impairment, and because it has been used in previous PWS studies (Clarke et al. 1996; Holland et al. 2003).

\section{Analysis}

The PWS and comparison group were compared with respect to performance on EF tasks and measures of behaviour. Because of the large number of comparisons made, the significance level for differences in performance on EF tasks was set at $P<0$. OI. As the hypothesis is directional, results are reported at a one-tailed level, however, results contrary to the hypothesis that show trends towards significance are reported as two-tailed.

\section{Differences in behaviour}

Mean scores and levels of significance (independent $t$-tests) are shown for the ABC and DEX in Table 2.
The PWS group had a significantly higher mean total ABC score than the comparison group $(P<0.05)$ and also showed significantly higher scores $(P<0.05)$ in factors 3 and 5, stereotypic behaviour and inappropriate speech. The PWS group had a higher mean score on the DEX, but it did not reach significance. In both the PWS and the comparison group, the ABC total score and the DEX were positively correlated $(P<0.01)$. There were no significant correlations between verbal IQ and the scores on the $\mathrm{ABC}$ and DEX for either the PWS or the comparison group. Table 3 shows data for the total ABC and DEX scores for the deletion ( $n=\mathrm{I} 2)$ and disomy $(n=6)$ groups. In this study the UPD sub-group had higher mean levels of behaviour problems but these differences were not significant.

\section{Differences in performance on EF tasks}

Table 4 shows the differences between the mean scores on EF tasks for the whole PWS and compar-

Table 2 Differences between mean scores on the behavioural questionnaires for the PWS and comparison group (independent $t$-tests, one tailed)

\begin{tabular}{|c|c|c|c|c|}
\hline & $\begin{array}{l}\text { PWS }(n=18) \\
\text { Mean (SD) }\end{array}$ & $\begin{array}{l}\text { Comparison }(n=15) \\
\text { Mean }(\mathrm{SD})\end{array}$ & $t$ & Significance \\
\hline \multicolumn{5}{|l|}{ Aberrant Behavior Checklist (ABC) } \\
\hline Factor I & $14.83(9.53)$ & $9.79(9.34)$ & 1.472 & 0.08 \\
\hline Factor 2 & $12.44(10.03)$ & $7.86(6.70)$ & 1.473 & 0.08 \\
\hline Factor 3 & $4.56(5.07)$ & $1.79(2.99)$ & 1.811 & $0.04 *$ \\
\hline Factor 4 & $5.78(7.41)$ & $8.00(8.16)$ & 0.808 & 0.21 \\
\hline Factor 5 & $4.72(3.21)$ & $2.43(2.59)$ & 2.174 & $0.02 *$ \\
\hline Total & $48.83(31.50)$ & $29.86(25.10)$ & 1.843 & $0.03^{*}$ \\
\hline Dysexecutive Questionnaire (DEX) & $44.50(17.46)$ & $38.43(14.87)$ & 1.039 & 0.15 \\
\hline
\end{tabular}

PWS, Prader-Willi syndrome.

$* P<0.05$.

Table 3 Differences between means of behavioural questionnaires and verbal IQ for deletion and UPD groups (independent $t$-tests, two tailed)

\begin{tabular}{lllll}
\hline & Deletion $(\boldsymbol{n}=\mathbf{1 2})(\mathrm{SD})$ & UPD $(\boldsymbol{n}=\mathbf{6})(\mathrm{SD})$ & $\boldsymbol{t}$ & Significance \\
\hline ABC total & $46.82(35.97)$ & $55.50(25.64)$ & 0.520 & 0.61 \\
DEX total & $43.09(19.01)$ & $50.00(14.56)$ & 0.771 & 0.45 \\
Verbal IQ & $71.64(12.08)$ & $76.50(8.64)$ & 0.867 & 0.40 \\
\hline
\end{tabular}

UPD, uniparental disomy, ABC, Aberrant Behavior Checklist; DEX, Dysexecutive Questionnaire.

(C) 2005 Blackwell Publishing Ltd, fournal of Intellectual Disability Research 49, 6r3-625 
R. M. Walley \& M. D. C. Donaldson - Executive function abilities in adults with PWS

Table 4 Differences between mean scores on EF tasks for the PWS and comparison groups (independent $t$-tests) ${ }^{t}$ denote results which show a trend towards significance and are contrary to the hypothesis, these results are reported as two tailed, otherwise all results are one tailed)

\begin{tabular}{|c|c|c|c|c|}
\hline & $\begin{array}{l}\text { PWS }(n=18) \\
\text { Mean (SD) }\end{array}$ & $\begin{array}{l}\text { Comparison } \\
(n=15) \\
\text { Mean (SD) }\end{array}$ & $t$ & Significance \\
\hline \multicolumn{5}{|l|}{ Initiating Behaviour } \\
\hline \multicolumn{5}{|l|}{ Verbal Fluency FAS } \\
\hline $\mathrm{F}$ & $3.8(3.2)$ & $5.7(4.8)$ & 1.384 & 0.08 \\
\hline A & $2.78(2.8)$ & $3.6(4.00)$ & 0.695 & 0.24 \\
\hline $\mathrm{S}$ & $4.9(3.6)$ & $5.1(4.5)$ & 1.333 & 0.44 \\
\hline Total FAS & $11.5(8.9)$ & $14.2(12.3)$ & 0.729 & 0.23 \\
\hline Repeated words & $0.28(0.67)$ & $0.20(0.56)$ & 0.357 & 0.36 \\
\hline Errors & $0.11(0.32)$ & $0.07(0.26)$ & 0.430 & 0.33 \\
\hline \multicolumn{5}{|l|}{ Categories } \\
\hline Animals & $11.2(4.5)$ & $11.2(5.1)$ & 0.020 & 0.49 \\
\hline Objects & $10.9(6.0)$ & $11.1(5.6)$ & 0.060 & 0.47 \\
\hline Occupations & $8.0(4.2)$ & $8.3(3.6)$ & 0.244 & 0.40 \\
\hline Categories total & $29.0(11.3)$ & $30.6(12.7)$ & 0.383 & 0.35 \\
\hline Category repeats & $0.67(1.02)$ & $0.40(0.91)$ & 0.781 & 0.22 \\
\hline Category errors & $0.0(0)$ & $0.13(0.52)$ & 1.099 & 0.14 \\
\hline \multicolumn{5}{|l|}{$\begin{array}{l}\text { Planning Ability } \\
\text { Tower of London (TOL) }\end{array}$} \\
\hline 3 & $1.94(0.87)$ & $1.60(1.06)$ & 1.027 & 0.15 \\
\hline 4 & $1.78(0.94)$ & $1.40(0.83)$ & 1.210 & 0.11 \\
\hline 5 & $1.11(0.76)$ & $0.53(0.64)$ & 2.336 & $0.02^{\dagger}$ \\
\hline 6 & $0.22(0.43)$ & $0.07(0.26)$ & 1.232 & 0.11 \\
\hline 7 & $0.22(0.55)$ & $0.20(0.56)$ & 0.115 & 0.45 \\
\hline Total & $5.28(1.71)$ & $3.8(2.31)$ & 2.113 & $0.04^{\dagger}$ \\
\hline \multicolumn{5}{|c|}{ TOL most complex problem solved correctly in minimum no. of moves } \\
\hline Block Design & $5.28(0.96)$ & $4.2(1.98)$ & 2.051 & $0.05^{t}$ \\
\hline Raw scores & $11.67(9.54)$ & $12.93(9.50)$ & 0.381 & 0.35 \\
\hline Age scaled score & $5.11(2.40)$ & $5.6(2.35)$ & 0.621 & 0.26 \\
\hline \multicolumn{5}{|l|}{ Set (rule) Changing Ability } \\
\hline Luria hand game no. of correct responses & $13.61(2.20)$ & $14.0(1.93)$ & 0.534 & 0.29 \\
\hline Rule Shift Cord test no. of errors on Rule 2 & $5.22(3.6)$ & $5.1(3.8)$ & 0.120 & 0.45 \\
\hline Spatial reversal task no. of sets (runs of 4 correct responses) & $2.94(1.11)$ & $2.47(1.55)$ & 1.029 & 0.15 \\
\hline \multicolumn{5}{|l|}{ Stroop - Day/Night Test } \\
\hline No. of errors in experimental condition & $1.89(4.03)$ & $2.20(3.65)$ & 0.230 & 0.40 \\
\hline No. of errors in control condition & $1.00(1.88)$ & $3.00(4.54)$ & 1.707 & 0.09 \\
\hline Time on experimental condition in seconds & $49.94(12.25)$ & $51.3(19.19)$ & 0.240 & 0.40 \\
\hline Time on control condition in seconds & $45.06(9.23)$ & $42.1(11.50)$ & 0.810 & 0.21 \\
\hline \multicolumn{5}{|l|}{ Working Memory } \\
\hline Total concrete pages before error & $30.89(3.32)$ & $30.40(5.17)$ & 0.329 & 0.37 \\
\hline Total abstract pages before error & $18.50(3.35)$ & $17.93(4.10)$ & 0.438 & 0.33 \\
\hline Total concrete position perseverations & $5.67(3.55)$ & $4.00(2.17)$ & 1.586 & 0.06 \\
\hline Total abstract position perseverations & $4.89(2.88)$ & $2.93(2.22)$ & 2.146 & 0.02 \\
\hline Total concrete item perseverations & $2.67(1.37)$ & $2.40(2.56)$ & 0.382 & 0.35 \\
\hline Total abstract item perseverations & $8.44(1.85)$ & $8.87(2.42)$ & 0.568 & 0.28 \\
\hline \multicolumn{5}{|l|}{ Digit Span } \\
\hline Max digits forward & $3.22(1.52)$ & $4.53(1.85)$ & 2.241 & $0.01 *$ \\
\hline Max digits backward & $2.17(1.15)$ & $2.33(1.80)$ & 0.322 & 0.37 \\
\hline
\end{tabular}

EF, executive function; PWS, Prader-Willi syndrome; UPD, uniparental disomy.

${ }^{*} P<0.01$.

'Trend towards significance contrary to hypothesis.

(1) 2005 Blackwell Publishing Ltd, fournal of Intellectual Disability Research 49, 6r3-625 
R. M. Walley \& M. D. C. Donaldson - Executive function abilities in adults with PWS

ison groups. No significant differences were found in performance on the EF tasks, however, a significant difference was observed in the non-executive Digit Span Forwards test.

Contrary to the predictions of the study the PWS group had better performance, although not reaching significance, on the 5 move, and the total correct problems made in the minimum number of moves on the TOL, as well as a higher mean score on the most complex TOL problem solved correctly (reported as two-tailed).

Table 5 shows the differences between means for the deletion and UPD sub-groups on the EF tasks.

There were no significant differences between the two sub-groups on any of the EF tasks or Digit Span Forwards.

Analyses of differences between the two subgroups and the comparison group on performance on the TOL and Digit Span Forwards are shown in Table 6. The deletion sub-group were more able at solving 5 move and more complex problems on the TOL than the UPD sub-group relative to the comparison group, although these findings did not reach significance.

A significant finding was that the deletion subgroup were poorer than the comparison group on Digit Span Forwards $(P<0.01)$. The UPD sub-group were poorer on this test than the comparison group but not significantly so.

\section{Discussion}

In common with previous studies (Clarke et al. I996; Holland et al. 2003) the PWS group had a much higher mean score on the $\mathrm{ABC}$ than the comparison group. The mean total score on the $\mathrm{ABC}$ was higher in the current study than in previous studies, but this may reflect a bias in recruitment; the sample in the Holland et al. study was widely drawn from a geographical region, whereas in the current study the participants were recruited from a PWS clinic. The PWS group had significantly higher scores on factors 3 - stereotypic behaviour, and 5-inappropriate speech. The mean scores for factors I - irritability and agitation, and 2 - lethargy and withdrawal, were higher in the PWS group and almost reached significance. In the Clarke et al. study, factors I and 5 were significantly higher in their PWS group, but in the
Holland et al. study only factor I was significantly higher than in a comparison group.

For both the PWS and comparison groups the DEX total scores were significantly correlated with the ABC total scores $(P<0.01)$, but the PWS total mean score on the DEX was not significantly higher than in the comparison group; this suggests that the behavioural difficulties in PWS may not be attributable to difficulties in EF alone. Interestingly in this study the UPD group were reported as having more behavioural problems than the deletion group.

Despite the prediction that there would be a number of relative EF deficits in the PWS group, no significant differences were found in performance on EF tasks.

Although not reaching significance the better performance of the PWS group, particularly the deletion sub-group, on some aspects of the planning task, the TOL is consistent with the observation of Dykens (2002) that individuals with the deletion sub-type show relative ability at visuospatial tasks.

A significant finding was the poorer performance of the PWS group, particularly those with a deletion, on a non-executive task, Digits Span Forwards, which is often used as a measure of the phonological loop (Conners et al. 1998). The difficulty the PWS group had on Digit Span Forwards was in contrast to their relatively good performance on the Selfordered pointing task, a visually presented test of working memory. Both the PWS and the comparison groups performed slightly better on this latter task than a group of younger participants with Turner syndrome and a comparison group who were all of average intelligence (Temple et al. 1996).

The relatively good performance of the PWS group on the Self-ordered pointing task, which is presented visually, compared with their difficulties on Digit Span Forwards, which is presented auditorally, underlines the importance of using visual strategies with this population. Considering the satisfactory performance of the PWS group on the Self-ordered pointing task, it may be that the difficulty in Digit Span Forwards lies not in the central executive process of working memory but rather in the capacity of the phonological store component of the articulatory loop. This possibility may be particularly salient for the deletion group and invites further investigation. Evidence from positron emission tomography (PET) studies suggest that the phonological loop is located 


\begin{tabular}{|c|c|c|c|c|}
\hline & $\begin{array}{l}\text { Deletion }(n=12) \\
\text { Mean }(\mathrm{SD})\end{array}$ & $\begin{array}{l}\text { UPD }(n=6) \\
\text { Mean (SD) }\end{array}$ & $t$ & Significance \\
\hline \multicolumn{5}{|l|}{ Initiating Behaviour } \\
\hline \multicolumn{5}{|l|}{ Verbal Fluency } \\
\hline $\mathrm{F}$ & $2.82(2.82)$ & $6.00(3.23)$ & 2.116 & 0.05 \\
\hline A & $2.36(2.25)$ & $4.00(3.58)$ & 1.167 & 0.26 \\
\hline $\mathrm{s}$ & $4.00(3.58)$ & $6.50(3.73)$ & 1.358 & 0.19 \\
\hline Total FAS & $9.18(8.12)$ & $16.50(9.85)$ & 1.65 & 0.12 \\
\hline Repeated words & $0.00(0.00)$ & $0.83(0.98)$ & 2.076 & 0.09 \\
\hline Errors & $0.09(0.30)$ & $0.17(0.41)$ & 0.438 & 0.67 \\
\hline \multicolumn{5}{|l|}{ Categories } \\
\hline Animals & $11.18(5.09)$ & $12.17(2.93)$ & 0.432 & 0.67 \\
\hline Objects & $9.91(5.84)$ & $13.67(6.22)$ & 1.241 & 0.23 \\
\hline Occupations & $6.73(3.04)$ & $10.33(5.54)$ & 1.756 & 0.09 \\
\hline Categories total & $27.82(12.21)$ & $32.83(9.79)$ & 0.862 & 0.40 \\
\hline Category repeats & $0.55(0.93)$ & $1.00(1.27)$ & 0.332 & 0.41 \\
\hline Category errors & $0.00(0.00)$ & $0.00(0.00)$ & - & - \\
\hline \multicolumn{5}{|l|}{ Planning Ability } \\
\hline \multicolumn{5}{|l|}{ Tower of London (TOL) } \\
\hline \multicolumn{5}{|c|}{ TOL no. of problems solved correctly in minimum no. of moves } \\
\hline 3 & $1.91(.83)$ & $2.17(0.98)$ & 0.574 & 0.57 \\
\hline 4 & $1.55(1.04)$ & $2.17(0.75)$ & 1.287 & 0.22 \\
\hline 5 & $1.18(0.75)$ & $1.00(0.89)$ & 0.447 & 0.66 \\
\hline 6 & $0.27(0.47)$ & $0.17(0.41)$ & 0.466 & 0.65 \\
\hline 7 & $0.18(0.41)$ & $0.33(0.82)$ & 0.519 & 0.61 \\
\hline Total & $5.09(1.30)$ & $5.83(2.40)$ & 0.838 & 0.42 \\
\hline $\begin{array}{l}\text { Mean of most complex problem solved correctly in } \\
\text { min no. of moves }\end{array}$ & $5.45(0.93)$ & $5.00(1.09)$ & 0.769 & 0.38 \\
\hline \multicolumn{5}{|l|}{ Block Design } \\
\hline Raw scores & $14.00(10.79)$ & $6.67(5.57)$ & 1.54 & 0.14 \\
\hline Age scaled score & $5.64(2.77)$ & $4.00(1.41)$ & 1.342 & 0.19 \\
\hline \multicolumn{5}{|l|}{ Set (Rule) Changing Ability } \\
\hline Luria Hand game no. of correct responses & $13.09(2.70)$ & $14.33(.52)$ & 1.100 & 0.28 \\
\hline Rule Shift Card test no. of errors on Rule 2 & $5.82(3.66)$ & $4.50(3.73)$ & 0.706 & 0.49 \\
\hline $\begin{array}{l}\text { Spatial reversal task no. of sets (runs of } 4 \text { correct } \\
\text { responses) }\end{array}$ & $3.18(1.08)$ & $2.50(1.23)$ & 1.189 & 0.25 \\
\hline \multicolumn{5}{|l|}{ Stroop - day/night test } \\
\hline No of errors in experimental condition & $1.64(3.35)$ & $2.67(5.57)$ & 0.480 & 0.63 \\
\hline No of errors in control condition & $0.82(2.08)$ & $1.33(1.75)$ & 0.512 & 0.61 \\
\hline Time on experimental condition in seconds & $52.27(13.36)$ & 45.67 (10.91) & 1.033 & 0.31 \\
\hline Time on control condition in seconds & $44.27(9.60)$ & $46.17(9.94)$ & 0.382 & 0.70 \\
\hline \multicolumn{5}{|l|}{ Working Memory } \\
\hline \multicolumn{5}{|l|}{ Self-ordered Pointing Test } \\
\hline Total concrete pages before error & $30.45(3.73)$ & $31.67(2.94)$ & 0.686 & 0.50 \\
\hline Total abstract pages before error & $19.00(4.19)$ & $17.83(1.17)$ & 0.658 & 0.52 \\
\hline Total concrete position preservations & $5.00(2.72)$ & $6.83(5.04)$ & 0.987 & 0.33 \\
\hline Total abstract position perseverations & $4.82(2.96)$ & $5.00(3.29)$ & 0.117 & 0.90 \\
\hline Total concrete item perseverations & $2.64(1.63)$ & $2.67(1.03)$ & 0.041 & 0.96 \\
\hline Total abstract item perseverations & $8.09(2.02)$ & $9.00(1.67)$ & 0.910 & 0.36 \\
\hline \multicolumn{5}{|l|}{ Digit Span } \\
\hline Max digits forward & $2.82(1.60)$ & $4.00(1.27)$ & 1.555 & 0.14 \\
\hline Max digits backward & $1.82(1.25)$ & $2.83(0.75)$ & 1.803 & 0.09 \\
\hline
\end{tabular}

EF, exccutive function; UPD, uniparental disomy. 
Table 6 Significance of mean differences for deletion and UPD groups compared with the comparison group, on the variables that were significant or showed a trend towards significance for the whole PWS group compared to comparison group (independent $t$-tests)

\begin{tabular}{lll}
\hline Variable & & Significance \\
\cline { 2 - 3 } & Deletion group $(n=12)$ & UPD group $(n=6)$ \\
Tower of London & & $0.02^{\dagger}$ \\
5 move problems solved in min moves & $0.10^{\dagger}$ & $0.08^{\dagger}$ \\
Total problems solved correctly in min moves & $0.06^{\dagger}$ & $0.36^{\dagger}$ \\
Most complex problems solved correctly in min moves & $0.01^{* \dagger}$ & $0.26^{\ddagger}$ \\
Digit Span Forwards & & \\
\hline
\end{tabular}

UPD, uniparental disomy; PWS, Prader-Willi syndrome.

${ }^{*} P<0.01$.

'Two-tailed, i.e. better performance than the comparison group contrary to experimental hypothesis.

tOne-tailed, i.e. poorer performance than the comparison group consistent with experimental hypothesis.

in the left parietal lobe (Paulesu et al. 1993), whereas maintenance of Digit Span Forward is carried out in the midventrolateral cortex (Owen et al. 2000).

Small numbers, especially of individuals with UPD, limit the findings of the study and the nonrepresentative selection of participants also means that the findings cannot be generalized to the whole PWS population. The results suggest, however, that further investigation of the phonological loop component of working memory is warranted in PWS to confirm or refute the findings of the present study and to investigate further, differences between the two genetic sub-types.

In conclusion, the only deficit found in the PWS group relative to the comparison group was not in an $\mathrm{EF}$ task but in a non-executive process Digit Span Forwards, which may possibly be because of impairment in the capacity of the phonological store. Further studies with larger numbers of participants are required but this impairment appears to be particularly salient for the deletion sub-type where it may contribute to more efficient processing of visuospatial tasks by placing greater reliance on the visuospatial sketchpad.

Although reliance on the visuospatial sketchpad may contribute to the behavioural problems found in PWS, it does not adequately account for their disproportionate level, as problems in the phonological loop are also reported in Down syndrome, which is not characterized by the same type or level of behavioural difficulties (Jarrold et al. 2000).
There is evidence that EF tasks have a greater association with dorsolateral as opposed to other parts of the pre-frontal cortex. Scanning studies have implicated the involvement of the dorsolateral cortex in the Tower of London Task (Baker et al. 1996; Cazalis et al. 2003), Self-ordered pointing and other working memory tasks are also believed to engage the dorsolateral cortex (Petrides I998).

The current study indicates that individuals with PWS do not have relative difficulty with tasks associated with the dorsolateral cortex and that in PWS another region of the pre-frontal cortex may be involved. One candidate could be the orbitofrontal cortex (OFC) and closely linked ventromedial cortex. The OFC projects to the limbic system including the hypothalamus, and is involved in the modulation of emotion. Problems in the OFC can give rise to abnormalities in the ability to engage the appropriate emotions in relation to social situations and events and may occur even when adequate social knowledge is available (Bechara et al. 2000; Elliot et al. 2000). Arrested development of the orbitofrontal cortex in PWS may interfere with the ability to inhibit inappropriate emotional responses to unexpected situations, e.g. a thwarted plan or routine, even though relatively good cognitive planning skills are present. Further indirect evidence that the orbitofrontal cortex may be implicated in the behaviours seen in PWS is provided by studies which show that this region of the brain is involved in compulsive behaviours (Volkow \& Fowler 2000; Choi et al. 2004), mood and affective disorders (Drevets 200I), and in applying 
verbal learning strategies in novel situations (Savage et al. 200I).

\section{References}

Aman M. G., Field C., Singh N. \& Stewart A. (1985) The Aberrant Behaviour Checklist: a behaviour rating scale for the assessment of treatment effects. American fournal of Mental Deficiency 89, 485-91.

Aman M. G. \& Singh N. (1994) Aberrant Behaviour Checklist-Community. Slosson Educational Publications, New York.

Baddeley A. D. (1986) Working Memory. Oxford University Press, Oxford.

Baddeley A. D. \& Hitch G. (1974) Working memory. In: Recent Advances in Learning and Motivation (ed. G. A. Bower), Vol. 8, pp. 647-67. Academic Press, New York.

Baker S., Rogers R., Owen A., Frith C., Dolan F., Frackowiak R. S. \& Robbins T. (1996) Neural systems engaged by planning: a PET study of the Tower of London Task. Neuropsychologia 34, 515-26.

Bechara A., Damasio H. \& Damasio A. (2000) Emotion, decision-making and the orbitofrontal cortex. Cerebral Cortex 10, 295-307.

Boer H., Holland A., Whittington J. E., Butler J. V., Webb T. \& Clarke D. J. (200I) Is psychotic illness inevitable in people with Prader-Willi syndrome due to chromosome I5 maternal uniparental disomy. Lancet 359, I35-6.

Burgess P. W. (1997) Theory and methodology in executive function research. In: Methodology of Frontal and Executive Function (ed. P. Rabbitt), pp. 81-116. Psychology Press, Hove, East Sussex.

Cazalis F., Valabregue R., Pelegini-Isaac M., Asloun S., Robbins T. W. \& Granon S. (2003) Individual differences in prefrontal cortical activation in the Tower of London planning task: implication for effortful processing. European fournal of Neuroscience 17, 2219-25.

Choi J.-S., Kang D.-H., Kim J.-J., Ha T.-H., Lee J.-M., Youn T., Kim I. Y., Kim S. I. \& Kwon J. S. (2004) Left anterior subregion of orbitofrontal cortex Volume reduction and impaired organisational strategies in obsessivecompulsive disorder. Fournal of Psychiatric Research 38, I93-9.

Clarke D. J., Boer H., Chung M. C., Sturmey P. \& Webb T. (1996) Maladaptive behaviour in Prader-Willi syndrome in adult life. Gournal of Intellectual Disability Research 40, 159-65.

Clarke D. J., Boer H., Whittington J., Holland A., Butler J. \& Webb T. (2002) Prader-Willi syndrome compulsive and ritualistic behaviours: the first population-based survey. British Gournal of Psychiatry 180, 358-62.

Conners F. A., Carr D. C. \& Willis S. (1998) Is the phonological loop responsible for intelligence-related differences in forward digit span? American fournal on Mental Retardation 103, I-11.
Culbertson W. \& Zillmer E. (I998) The Tower of London Drexel Version: a standardized approach to assessing executive functioning in children. Archives of Clinical Neuropsychology 13, 285-301.

Cummings J. (1993) Frontal-sub cortical circuits and human behaviour. Archives of Neurology 50, 873-80.

Curfs L. M. G., Wiegers A. M., Sommers J. R. M. Borghgraef M. \& Fryns J. P. (199I) Strengths and weaknesses in the cognitive profile of youngsters with Prader-Willi syndrome. Clinical Genetics 40, 430-4.

Denckla M. B. (1996) A theory and model of executive function: a neuropsychological perspective. In: Attention, Memory and Executive Function (eds G. R. Lyon \& N. A. Krasnegor), pp. 263-78. Paul Brookes, Baltimore, MD.

Drevets W. C. (200I) Neuroimaging and neuropathological studies of depression: implications for the cognitiveemotional features of mood disorders. Current Opinion in Neurobiology II, 240-9.

Dykens E. M. (2002) Are jigsaw puzzle skills 'spared' in persons with Prader-Willi syndrome? fournal of Child Psychology and Psychiatry 43, 343-52.

Dykens E., Cassidy S. \& King B. (1999) Maladaptive behavior differences in Prader-Willi syndrome due to paternal deletion versus maternal uniparental disomy. American Journal on Mental Retardation r04, 67-7.

Dykens E., Hodapp R., Walsh K. \& Nash L. (I992a) Profiles, correlates and trajectories of intelligence in individuals with Prader-Willi syndrome. Fournal of the American Academy of Child and Adolescent Psychiatry 31, I125-30.

Dykens E., Hodapp R., Walsh K. \& Nash L. (1992b) Adaptive and maladaptive behavior in Prader-Willi syndrome. fournal of the American Academy of Child and Adolescent Psychiatry 31, 1131-5.

Dykens E. \& Kasari C. (1997) Maladaptive behavior in children with Prader-Willi syndrome, Down syndrome and Non-specific mental retardation. American fournal on Mental Retardation 102, 228-37.

Einfeld S., Smith A., Durvasula S., Florio T. \& Tonge B. (1999) Behavior and emotional disturbance in PraderWilli syndrome. American fournal of Medical Genetics 82, 123-7.

Elliot R., Dolan R. J. \& Frith C. D. (2000) Dissociable functions in the medial and lateral orbitofrontal cortex: evidence from human neuroimaging studies. Cerebral Cortex 10, 308-17.

Gerstadt C., Hong Y. J. \& Diamond A. (1994) The relationship between cognition and action: performance of children $3^{\mathrm{I} / 2}-7$ years old on a Stroop-like day-night test. Cognition 5, 129-53.

Holland A. J., Whittington J. E., Butler J., Webb T., Boer H. \& Clarke D. (2003) Behavioural phenotypes associated with specific genetic disorders: evidence from a population-based study of people with Prader-Willi syndrome. Psychological Medicine 33, 14I-53. 
R. M. Walley \& M. D. C. Donaldson - Executive function abilities in adults with PWS

Holm V., Cassidy S. B., Butler M. G., Hanchett J. M., Greenswag L. R., Whitman B. Y. \& Greenberg F. (1993) Prader-Willi syndrome: consensus diagnostic criteria. Pediatrics 9r, 398-402.

Hughes C. (1996) Control of action and thought: normal development and dysfunction in autism: a research note. fournal of Child Psychology and Psychiatry 37, 229-36.

Hughes C. \& Graham A. (2002) Measuring executive functions in childhood: problems and solutions? Child and Adolescent Mental Health 7, 13 I-42.

Jarrold C., Baddeley A. D. \& Hewes A. K. (2000) Verbal short-term memory deficits in Down syndrome: a consequence of problems in rehearsal? Fournal of Child Psychology and Psychiatry 40, 233-44.

MacEvoy R., Rogers S. \& Pennington B. F. (1993) Executive function and social communication deficits in young autistic children. Fournal of Child Psychology and Psychiatry 34, 563-78.

Newcombe F. (1969) Missile Wounds of the Brain: A Study of Psychological Deficits. Oxford University Press, Oxford.

Owen A. M., Lee A. C. H. \& Williams E. J. (2000) Dissociating aspects of verbal working memory within the human frontal lobe: further evidence for a 'process specific' model of lateral frontal organisation. Psychobiology 28, 146-55.

Paulesu E., Frith C. D. \& Frackowiak R. S. J. (1993) The neural correlates of the verbal component of working memory. Nature 362, 342-3.

Pennington B. F. \& Ozonoff S. (1996) Executive function and developmental psychopathology. Fournal of Child Psychology and Psychiatry 37, 51-88.

Petrides M. (1998) Specialised systems for the processing of mnemonic information within the primate frontal cortex. In: The Prefrontal Cortex Executive and Cognitive Functions (eds A. C.Roberts, T. W. Robbins \& L. Weiskrantz), pp. 103-16. Oxford University Press, Oxford.

Petrides M. \& Milner B. (1982) Deficits in subject-ordered tasks after frontal and temporal lobe lesions in man. Neuropsychologia 20, 249-62.

Roof E., Stone W., MacLean W., Feurer I., Thompson T. \& Butler M. (2000) Intellectual characteristics of PraderWilli syndrome: a comparison of genetic subtypes. fournal of Intellectual Disability Research 44, 25-30.

Rosenquist C., Conners F. A. \& Roskos-Ewoldsen B. (2003) Phonological and visuospatial working memory in individuals with intellectual disability. American fournal on Mental Retardation 6, 403-13.

Savage C. R., Deckersbach T., Heckers S., Wagner A. D., Schacter D. L. \& Alpert N. M. (200I) Prefrontal regions supporting spontaneous and directed application of verbal learning strategies, evidenced from PET. Brain 124, 219-3I.

Shallice T. (1982) Specific impairments of planning. Philosophical Transactions of the Royal Society of London B 298, 199-209.
Sparrow S., Balla D. \& Cicchetti D. (1984) Vineland Adaptive Behavior Scales. NFER - Nelson, Windsor.

State M., Dykens E., Rosner B., Martin A. \& King B. (I999) Obsessive-compulsive symptoms in Prader-Willi and 'Prader-Willi like' patients. Fournal of the American Academy of Child and Adoloscent Psychiatry 38, 329-34.

Temple C., Carney R. \& Mullarkey S. (1996) Frontal lobe function and executive skills in children with Turner's syndrome. Developmental Neuropsychology r2, 343-63.

Volkow N. D. \& Fowler J. S. (2000) Addiction, a disease of compulsion and drive: involvement of the orbitofrontal cortex. Cerebral Cortex 10, 318-25.

Waters J. (1999) Prader-Willi syndrome. A Practical Guide. David Fulton, London.

Webb T., Whittington J., Boer H., Clarke D., Butler J. \& Holland A. (2002) A study of the influence of different genotypes on the physical and behavioural phenotypes of children and adults ascertained clinically as having PWS. Clinical Genetics 62, 273-81.

Wechsler D. (1981) Wechsler Adult Intelligence Scale - Revised. Psychological Corporation, London.

Whittington J., Holland A., Webb T., Butler J., Clarke D. \& Boer H. (2004a) Cognitive abilities and genotype in a population based sample of people with Prader-Willi syndrome. Journal of Intellectual Disability Rescarch 48, 17287.

Whittington J., Holland A., Webb T., Butler J., Clarke D. \& Boer H. (2004b) Academic underachievement by people with Prader-Willi syndrome. Fournal of Intellectual Disability Research 48, 188-200.

Wigren M. \& Hansen S. (2003) Rituals and compulsivity in Prader-Willi syndrome: profile and stability. Fournal of Intellectual Disability Research 47, 428-38.

Wilson B., Alderman N., Burgess P., Emslie H. \& Evans J. (1996) Behavioural Assessment of the Dysexecutive Syndrome (BADS). Thames Valley Test Company, Suffolk, UK.

\section{Appendix}

\section{Initiating Behaviour}

\section{Verbal Fluency}

Two variations of this task were given to participants. The first was a traditional verbal fluency FAS task (Newcombe 1969); participants were asked to produce as many words as they could beginning with the letters F, A and S in a minute. Participants were given a score for the number of words produced for each letter and from that a total score was derived. The number of errors, and number of words that were repeated were also recorded. Participants were also asked to name as many items as possible from three 
R. M. Walley \& M. D. C. Donaldson - Executive function abilities in adults with PWS

semantic categories, a minute was allowed for each category; the categories were animals, objects and occupations (Temple et al. 1996).

\section{Planning Ability}

Tower of London (TOL)

A standardized adaptation of the TOL task (Shallice I982) was used to assess planning ability, the TOL Drexel Version (Culbertson \& Zillmer 1998). This comprises a board on which there are three vertical pegs placed equally apart. The pegs are of different sizes, on which can be placed, in the case of the largest peg, three coloured blocks - green, red and blue, with holes through them. The other two pegs can take two and one blocks respectively. The task required the participants to move the blocks from an initial starting position, which is constant for all problems, to an end position illustrated on a sheet of paper, one for each problem. Keeping to standardized rules participants had to move the blocks in a minimum number of moves; problems increased in difficulty from those, which could be done in a minimum of three moves to problems that could only be done in a minimum of seven moves. Each level of move had three different problems, giving a total of I5 problems altogether. Participants were given three practice two-move problems, in order to help them understand what they had to do, and were then given up to 2 minutes to solve each problem. The number of problems correctly solved in the minimum number of moves within the allotted time limit provided a score. In order to control for difficulties in visuospatial ability, participants were also given the Block Design sub-test from the Wechsler Adult Intelligence Scale - Revised (WAIS-R).

\section{Set/Rule Changing Ability and Inhibition of a Pre-Potent Response}

\section{Luria Hand Game Adapted by Hughes (1996)}

In this task participants were taught a simple game of motor inhibition. They were first taught to model two hand-gestures, a pointed finger and a clenched fist, and were then required to produce the opposite gesture shown by the tester, i.e. if the tester produced a clenched fist, the participant was expected to demonstrate a pointed finger. Participants had to inhibit the natural response to imitate the tester. Each participant was given 15 trials.

\section{Rule Shift Card Test - Wilson et al. (1996)}

This test, which is part of the Behavioural Assessment of Dysexecutive Syndrome (BADS; Wilson et al. I996) used 2I spiral bound, non-picture playing cards, and investigated participants' ability to respond correctly to a rule and then to shift from that rule to another one. In the first part of the task participants were asked to say 'yes' when they saw a red card, and 'no' when they saw a black card. This rule was printed on a card that was left in full view throughout the test to reduce memory constraints. In the second part of the test participants were asked to respond 'yes' if the card that had just been turned over was the same colour as the previously turned card, and 'no' if it was a different colour. The challenge in the second part of the task was therefore to concentrate on applying the new rule. As in the first part of the task, the new rule was left in full view of the participant. If a participant was unable to read the rules, the tester read them until the participant was able to recall what the rule was. Recall of the rule was checked at the end of each part of the test. The number of correct responses was recorded.

\section{Spatial Reversal Task - MacEvoy et al. (1993)}

This task was developed to investigate EF in children with Autistic Spectrum Disorder, and was slightly adapted in this study. Participants were asked to search for an item (a coin) that was hidden under one of two cups; the cups were initially screened from the participant so they were not aware where the coin had been placed. After each search the screen was put back but the position of the coin was not changed until the participant was able to find it on four consecutive occasions. Its position was then changed to under the other cup and the process repeated. A total of 25 trials in total were given, and the number of set changes (i.e. runs of four) successfully completed was recorded.

\section{Stroop Type Task - Day/Night Version}

As the adult version of the Stroop Test requires fluent reading ability, the day/night Stroop Task developed by Diamond and colleagues (Gerstadt et al. 1994) 
R. M. Walley \& M. D. C. Donaldson - Executive function abilities in adults with PWS

was used. In this task, participants were asked to say 'night' when they saw a picture of the sun drawn on a white card, and 'day' when they saw a picture of moon and stars drawn on a black card. After being given a brief practice trial, participants were then shown a sequence of 16 cards arranged in a set order. Participants were also given a control version of the task in which they were taught to associate two separate abstract drawings with the words 'night' and 'day' and then again after a short practice given a trial of 16 cards in an arranged order. The time taken and the number of trials where the correct response was given were recorded.

\section{Working memory}

Self-ordered Pointing Test - Temple et al. (1996)

In this task, which is an adaptation of a self-ordered pointing task devised by Petrides and Milner (1982), participants using their own strategies, had to remember the sequence in which they pointed to a set of visually displayed stimuli. The task has two versions both of which were given to participants; one uses black and white abstract designs and the other representational (concrete) black and white drawings of everyday objects. Each version of the test comprises sets of six, eight, Io and 12 pages.
On each page there are an equivalent number of designs or drawings. The designs/drawings in each set are the same but are in different spatial positions on each page. The participants were asked to point to a different design/drawing each time a page was turned. Scores were based on the number of pages correctly completed before an error was made, the number of times the same item was pointed to in a set (item preservation), and the number of repeated points made to the same spatial position (position preservation). Overall total scores were obtained, as well as separate scores for each set of stimuli.

\section{Digit span from the WAIS-R (Wechsler 1981)}

As auditory processing problems are reported in PWS, participants were given this sub-test from the WAIS-R, which is in two parts. Digits Forward is a useful measure of auditory attention and requires participants to recall strings of digits, which increase in length by one after every second presentation. In Digits Backward, participants have to recall similarly increasing strings of digits in reverse order. The maximum number of digits separately recalled forward and backwards was recorded.

Accepted I6 March 2005 